\title{
REPORT
}

\section{TECHNOLOGICAL EVALUATION \\ OF THE \\ DRILLING INDUSTRY \\ AND ITS \\ LONG RANGE REQUIREMENTS}
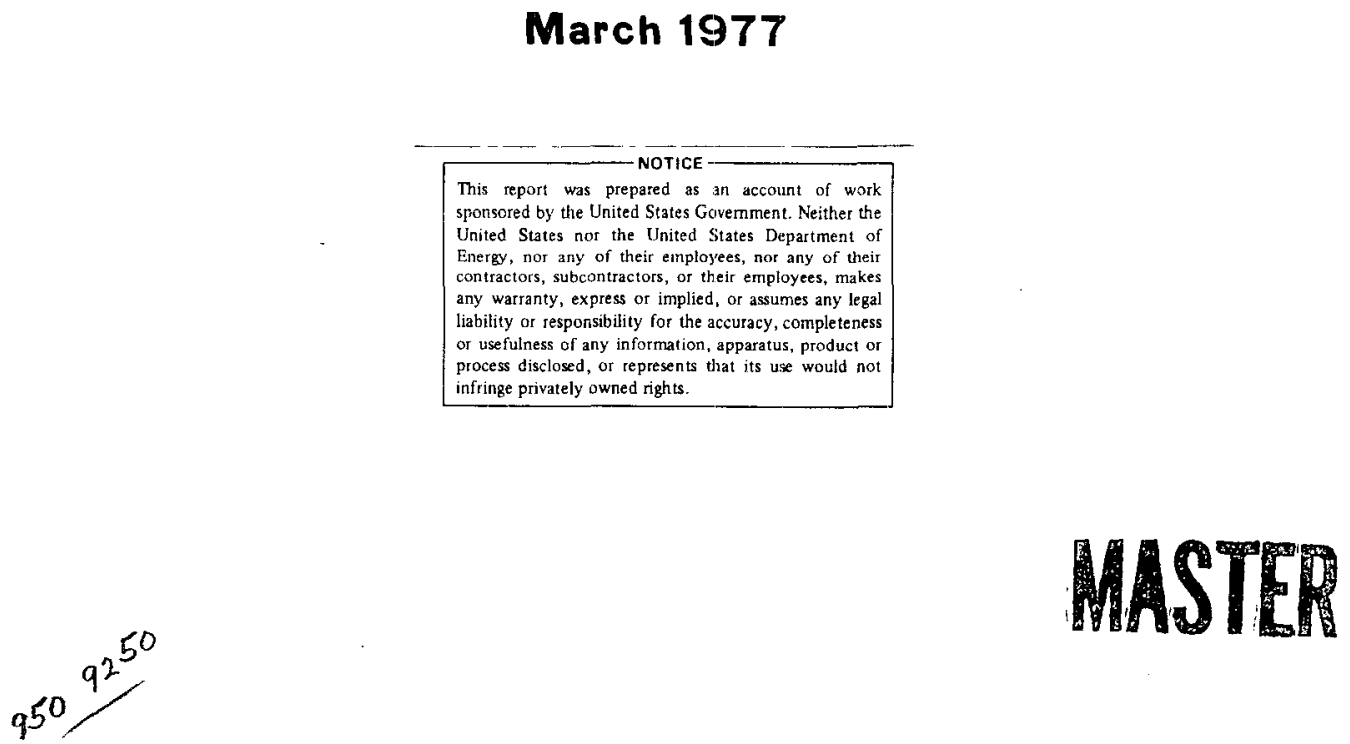

\section{WILLIAMS BROTHERS ENGINEERING COMPANY

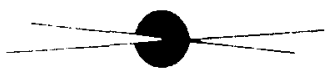 \\ A Resource Sciences company}

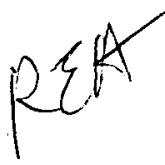

DISTRIBUTION OF TRLS DOCUMENT IS UNLIROTMD 


\section{DISCLAIMER}

This report was prepared as an account of work sponsored by an agency of the United States Government. Neither the United States Government nor any agency Thereof, nor any of their employees, makes any warranty, express or implied, or assumes any legal liability or responsibility for the accuracy, completeness, or usefulness of any information, apparatus, product, or process disclosed, or represents that its use would not infringe privately owned rights. Reference herein to any specific commercial product, process, or service by trade name, trademark, manufacturer, or otherwise does not necessarily constitute or imply its endorsement, recommendation, or favoring by the United States Government or any agency thereof. The views and opinions of authors expressed herein do not necessarily state or reflect those of the United States Government or any agency thereof. 


\section{DISCLAIMER}

Portions of this document may be illegible in electronic image products. Images are produced from the best available original document. 


\section{TABLE OF CONTENTS}

\begin{tabular}{|c|c|}
\hline Section & Title \\
\hline 1 & statement of Goals and objectives \\
\hline 2 & The Need for a Drilling Technology Program \\
\hline 3 & $\begin{array}{l}\text { Methodology Used to Select and Structure a } \\
\text { Drilling Technology Program }\end{array}$ \\
\hline 4 & Project Time-Cost Analysis \\
\hline 5 & Project Benefit Analysis \\
\hline Appendix & A - Drilling Company Analysis \\
\hline
\end{tabular}




\section{LIST OF TABLES}

Table

Title

$\underline{\text { Page }}$

2-1 Number of Rotary and Cable Drilling Rigs 2-2 in Operation 1950-1975

$2-2$

Survey of Drilling Companies Performing $2-3$ Contract Drilling for Major and Independent Oil Companies

$3-1$

Proposed Drilling Technology Program

Projects

$4-1$

Project 1-1, Training

$4-4$

$4-2$

Project 2-1, Upgrading Conventional Drilling 4-6 Rigs

$4-3$

Project 2-2, Drilling Rig Automation

$4-8$

$4-4$

Project

$2-3, D$

Directional

Drilling Equipment

$4-10$

$4-5$

Project 2-4, Bottom Hole Assemblies

$4-12$

$4-6$

Project

2-5, Mud Automation systems

$4-14$

$4-7$

Project

$3-1$

Rig

Instrumentation/Sensing

Equipment

$4-16$ 


\section{List of Tables (Continued)}

$4-8$

Project 3-2, Rig Flood Data Display/Analysis 4-18 Equipment

$4-9$

Project 3-3, Down-Hole Logging-Telemetry $4-20$ system

$4-10$

Project 3-4, Surface Logging-Telemetry $4-22$ systems 
List of Tables (Continued)

$5-5$

$5-6$

$5-7$

$5-8$

$5-9$

$5-10$

Project 2-5, Potential

5-11 Project 3-I, Potential

5-12 Project 3-2, Potential

5-13 Project 3-3, Potential

5-14 Project 3-4, Potential

$5-15$

Project 3-5, Potential

$5-16$

Project 4-1, Potential

5-17 Project 5-1, Potential

5-18 Project 6-1, Potential

$5-19$

Project 7-1, Potential

$5-20$

Project 1-1, Potential
$5-8$

$5-9$

$5-10$

5-11

$5-12$

$5-13$

5-14

$5-15$

$5-16$

$5-17$

$5-18$

$5-19$

$5-20$

$5-21$

$5-22$

$5-23$ 


\section{LIST OF FIGURES}

Figure

4-1
Title

Project Time-Cost Analysis
Page

$4-2$ 


\section{SECTION 1 \\ STATEMENT OF GOALS AND OBJECTIVES}

\section{Purpose}

The purpose of this study is to evaluate the drilling equiprent industry and to determine oil field drilling equipment products that should be considered for a development program.

\section{Goals and Objectives}

The primary goals and objectives of the study are:

1. List oil field equipment products suitable for development.

2. Determine cost/time factors involved in development of selected products.

3. Define product benefit factors in terms of improved drilling rig productivity (increased drilling penetration rate). 


\section{SECTION 2}

\section{THE NEED FOR A DRILLING TECHNOLOGY PROGRAM}

Higher oil and gas prices provide the drilling industry with an incentive to add new rigs and equipment and to drill additional wells.

Due to financial pressures caused by industry decline during the mid $1950^{\prime} \mathrm{s}$, competition for drilling contracts was strong. Drilling costs remained fairly constant and little funding was available for major research programs concerning improved drilling technology. This condition lasted until the mid-70's at which time a turn-around occurred.

During the period of peak activity prior to the mid 1950's, drilling equipment consisted of an even mix of cable rig drilling equipment and rotary drilling equipment. Since that time, the number of cable drilling rigs has declined substantially and now industry is using rotary drilling rigs; almost exclusively (Table 2-1). The application of new technology noted in this report refers only to rotary drilling rigs. The cable drilling method is considered outmoded for purposes of this study.

The majority of companies involved in the drilling industry are small to medium in size with only a few large companies (Table 2-2). Large companies concentrate primarily on offshore drilling and deep land drilling. The small to medium size companies concentrate primarily on land drilling. In general, they do not have sufficient economic strength to try unproven, new technologies or advance designed equipment. If the small to medium size company tried unproven technology 


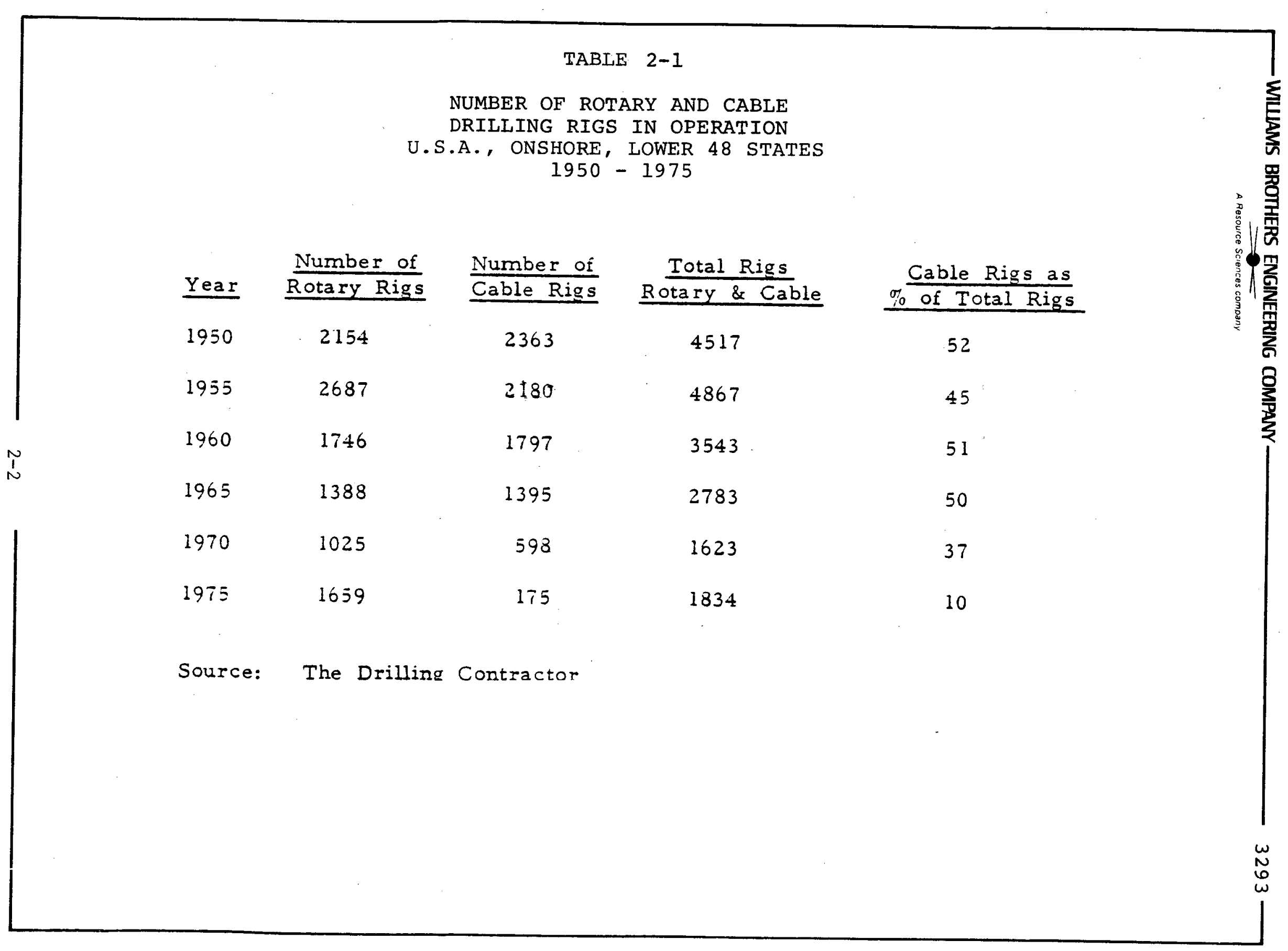


TABLE 2-2

SURVEY OF DRILLING COMPANIES

PERFORMING CONTRACT DRILLING FOR

MAJOR \& INDEPENDENT OIL COMPANIES

(1977 DATA)

Of 535 companies surveyed:

$25 \%$ have only 1 rig

$56 \%$ have 3 rigs or less

$73 \%$ have 5 rigs or less

$92 \%$ have 10 rigs or less

Excluded are companies with:
a. workover rigs only
b. foreign location of rigs

\section{Sources: Appendix A}

International Association of Drilling Contractors - 1977 Membership Directory

1977 Oil \& Gas Journal. Directory - Land Drilling \& Oil Well. Servicing Contractors. 
or equipment that performed improperly or failed completely, serious economic problems could result. Therefore, use of new technology is limited to large companies with numerous rigs and with the economic strength to experiment.

Since this market is limited, manufacturers associated with the drilling industry hesitate to advance new technologies. Thus, new technology advances slowly. Within this limited market, however, are a number of products that should be reviewed for development. Recommendations will be based on product ability to improve rig productivity or efficiency. Increased rig productivity or efficiency would provide the necessary economic incentive to justify purchase of the product. This study relates primarily to products that can provide this type of incentive. 


\author{
SECTION 3 \\ METHODOLOGY USED TO SELECT AND STRUCTURE \\ A DRILLING TECHNOLOGY PROGRAM
}

A study of existing drilling equipment capabilities provided data to determine innovations needed by the industry and segments of the market to which they are applicable. In addition industry literature was reviewed to evaluate emerging technologies and to determine their worth. These technologies and innovations were then reviewed with industry experts to establish their anticipated value and marketability to the drilling industry. From this analysis a list of projects was compiled. These projects are listed in Table 3-1.

Each project was reviewed and analyzed to establish:

1--Project Definition

2--Project Development Time and Cost Factors

3--Project Potential 
TABLE $3-1$

PROPOSED DRILLING TECHNOLOGY PROGRAM PROJECTS

1. Technology Transfer

Project 1 - Training

2. Drilling Equipment

Project 1 - Upgrading Conventional Drilling Rigs

Project 2 - Drilling Rig Automation

Project 3 - Directional Drilling Equipment

Project 4 - Bottom-Hole Assemblies

Project 5 - Mud Automation Systems

3. Drilling optimization

Project 1 - Rig Instrumentation/Sensing Equipment Project 2 - Rig Floor Data Display/Analysis Equipment Project 3 - Down-Hole Logging - Telemetry System

Project 4 - Surface Logging - Telemetry System Project 5 - Computer Controlled Drilling

4. High Pressure Drilling

Project 1 - Jet Erosion Drilling

5. Down-Hole Motors

Project 1 - Down-Hole Motor Technology 


$$
\text { Table 3-1, Continued }
$$

6. Drilling Bit Technology

Project 1 - stratapax and Bit Technology

7. BOP Technology

Project 1 - Blowout Preventer Equipment

8. Advanced Drilling Technology

Project 1 - Advanced Drilling Equipment 


\author{
SECIION 4 \\ PROJECT TIME-COST ANALYSIS
}

The selected drilling projects (Table 3-1) were analyzed to determine individual time-cost factors and to determine the cost effectiveness of the total program. To obtain this information, each project was reviewed as follows:

1. Project Definition
a. Project Description
b. State of the Art
c. Project Application

2. Project Development
a. Project Requirements
b. Project Time Duration
c. Project cost

Using the resulting data, the projects and time-cost factors were bar-graphed (Figure 4-1). As some projects are dependent upon the completion of segments of other projects, time phasing was analyzed and schedules set accordingly. Both yearly and total project costs were noted on the bar chart. 
Project 1-1 - Training (Table 4-1)

Description: Drilling company representatives, industry institutions and organizations, and the industry in general, recognize the need for increased training of its personnel. This need has been caused by the rapid expansion of the industry, the increased technical nature of equipment, and the in-general low level education of the average field worker. Lack of trained personnel is fundamental to the slow technological advancement of the industry.

An evaluation of training needs, recommendations for necessary instruction, and a program to apply these recommendations is required.

State of the Art: IADC and API sponsor schools with appropriate training aids and equipment. Company sponsored schools are increasing. However, industry use of these schools is relatively low.

Application: Training in all phases of drilling technology for personnel from all segments of the drilling industry is essential. 

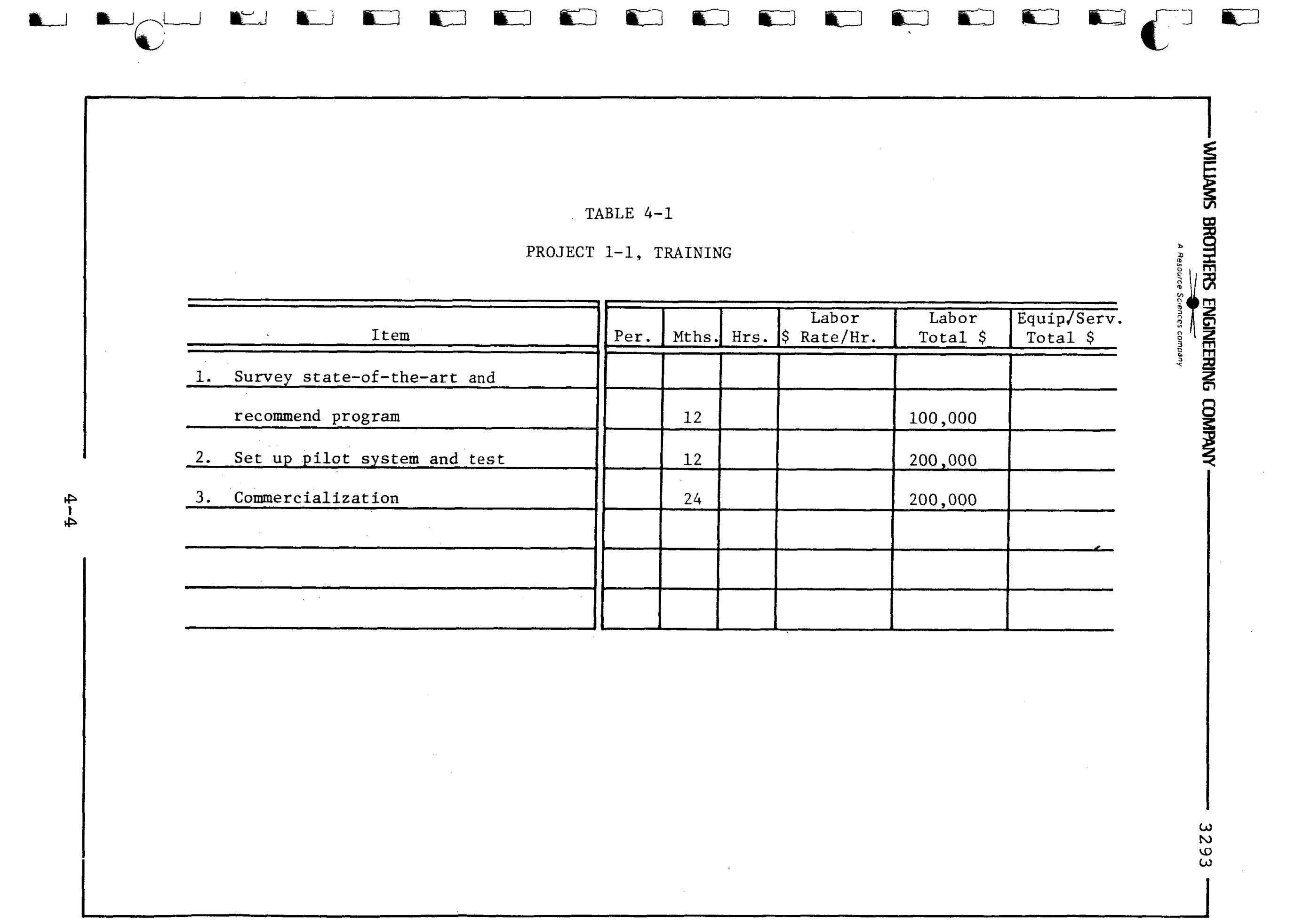


\section{Project 2-1 - Upgrading Conventional Drilling Rigs (Table 4-2)}

Description: The drilling industry has approximately 1,900 drilling rigs of various types and sizes. New and advanced industry technologies will not be adaptable to all present rigs. However, when possible, new innovative products (pipe: rackers, slips, elevators, tongs, etc.) should be built on a modular basis for retrofit into existing rigs. Project 2-1 is designed to evaluate conventional drilling rig obsolence and determine what can be done to alleviate it. Methods will be determined and field tested. The drilling industry will not scrap present rig fleets to buy new rigs, but will continue drilling with existing, outdated rigs or upgrade the equipment depending on their economic ability.

State of the Art: No studies have been made in this area. Implementation of Project $1-1$ and the phasing in of new techniques will affect the timing and strategy for Project 2-1.

Application: This project applies to all drilling rigs. 
TABLE 4-2

PROJECT 2-1, UPGRADING CONVENTIONAL DRILLING RIGS

\begin{tabular}{l|r|c|c|c|c|c|c}
\hline \multicolumn{1}{|c|}{ Item } & Per. & Meths. & Hrs. & $\begin{array}{c}\text { Labor } \\
\text { Rate/Hr. }\end{array}$ & $\begin{array}{c}\text { Labor } \\
\text { Total } \$\end{array}$ & $\begin{array}{c}\text { Equip/Serv } \\
\text { Total } \$\end{array}$ \\
\hline \hline 1. State-of-the-art & & 12 & & & 125,000 & \\
\hline 2. Conceptual & & 12 & & & 300,000 & \\
\hline 3. Initial Design & & 24 & & & 600,000 & \\
\hline 4. Lab/Field Test & & 24 & & & $1,000,000$ & $1,500,000$ \\
\hline 5. Commercialization & & 36 & & & 300,000 & \\
\hline
\end{tabular}

\begin{tabular}{l}
$w$ \\
$N$ \\
$\stackrel{\omega}{\omega}$ \\
\\
\hline
\end{tabular} 
Project 2-2 - Drilling Rig Automation (Table 4-3)

Description: After the conceptual/initial design phase of Project 2-1 is underway, project 2-2 will use the design information to evaluate Drilling Automation and field testing of equipment to determine its feasibility. Previous work has demonstrated that the automation concept is realistic and that it should improve the drilling penetration rate, down time, rig safety, control, and reduce manpower requirements. Automation will be a vital link in the overall concept of total computerized drilling. Design should take advantage of jet erosion drilling, down-hole telemetry, and down-hole drilling.

State of the Art: Automatic Drilling Machines, Dallas, Texas, has built and field-tested two automated drilling machines. Other companies have tried experimental automation systems. The concept has never been fully proven.

Application: This concept will apply to heavier, deeper rigs and to offshore and arctic applications. 


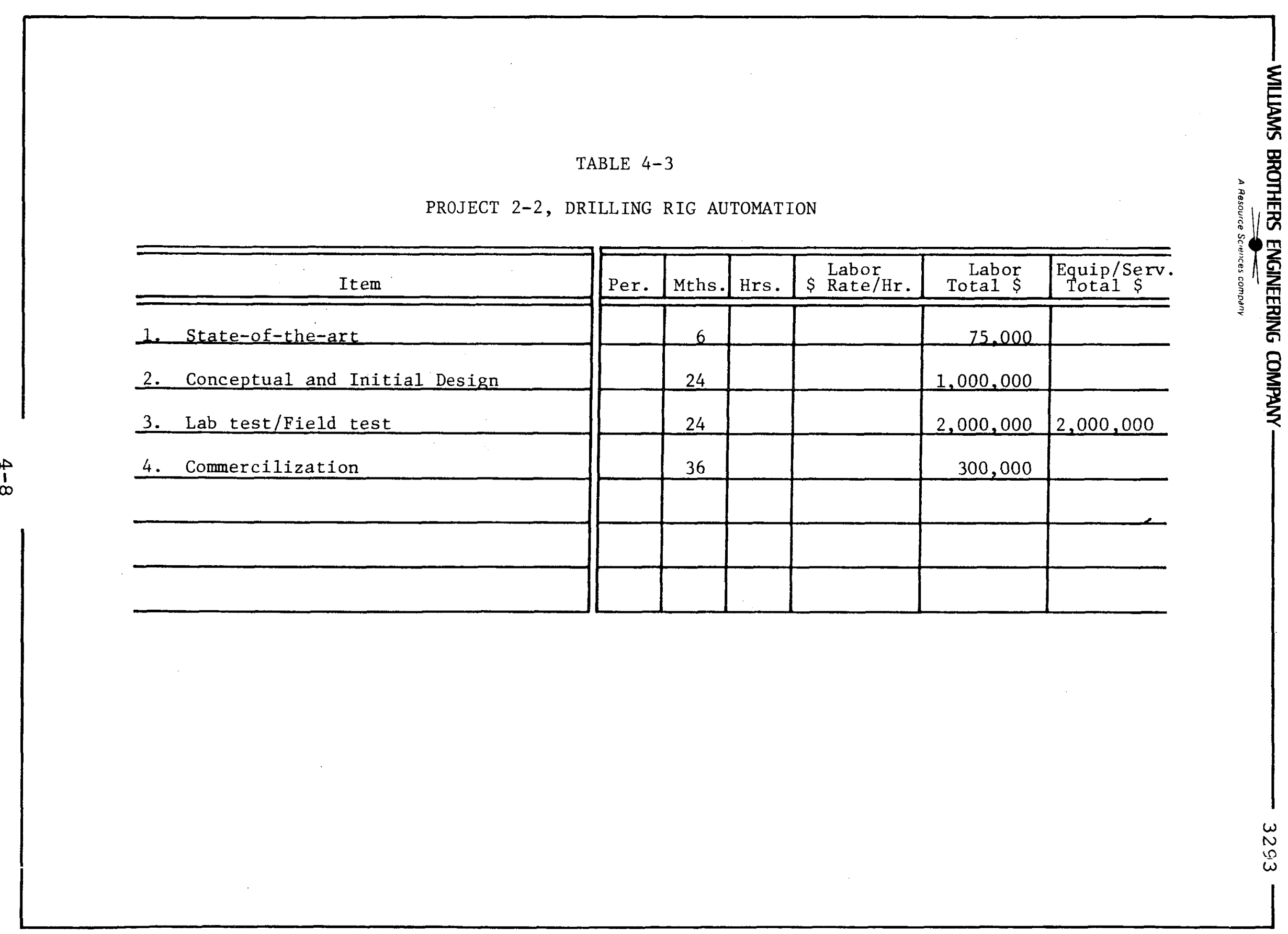




\section{Project 2-3 - Directional Drilling Equipment (Table 4-4)}

Description: This project will consist of evaluation, design assistance, and field testing of several integrated drilling systems/components to advance the technology level in directional drilling.

State of the Art: Industry has worked on various components; involved but little has been done to integrate these components into an overall system.

Application: This technology applies to all drilling when the rig is confined to a drilling pad or platform, such as offshore, arctic, or jungle drilling. It could be applied to advance exploration drilling concepts. 


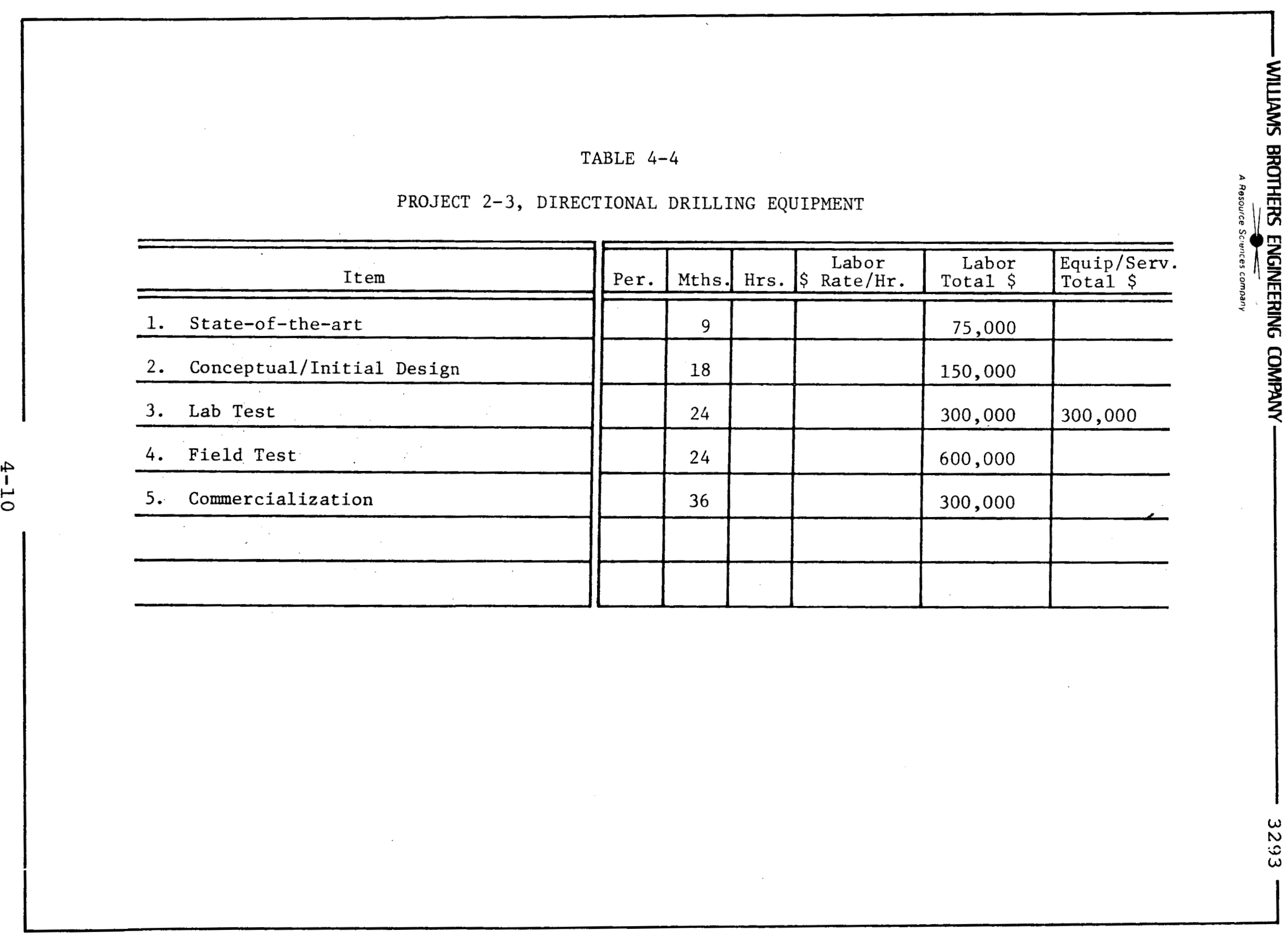


Project 2-4 - Bottom-Hole Assemblies (Table 4-5)

Description: The addition of stabilizers, collars, and other down-hole drilling components, if applied properly, can assist in determining the bit drilling direction, improve bit performance and bit life, and reduce drilling costs. Previous work indicates that the proper combination of components can be attained by use of mathematical modeling. Project 2-4 will evaluate pertinent technology and assist in its advancement and field use. Project 2-4 should begin immediately and should emphasize commercialization.

State of the Art: Amoco claims to have bottom-hole assemblies fully developed with excellent results. Christenson (B. Walker) claims bottom-hole assembly service is being marketed to European companies, but is just getting started in the U.S. All work done so far has applied to hole 10,000 feet and deeper.

Application: This technology could apply to drilling in general and will definitely apply to directional drilling. 


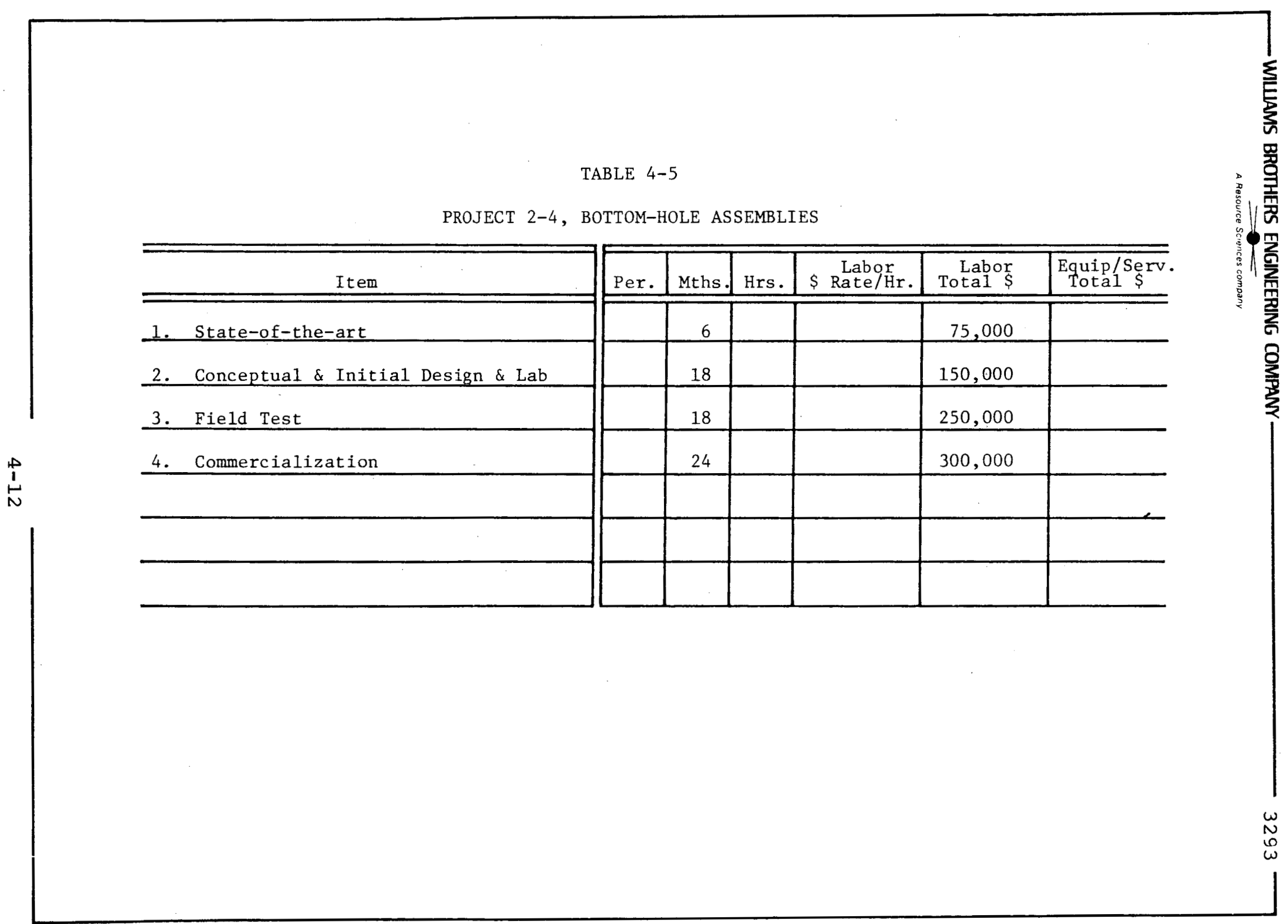


Project 2-5 - Mud Automation System (Table 4-6)

Description: Proper mud control automation systems can enhance drilling safety with reference to blowouts, and improve drilling penetration rates. This project will evaluate mud control automation and assist in developing, field testing, and advancing the concept if it is economically feasible. Project $2-5$ will be given low priority and should be undertaken on a go/no-go basis.

State of the Art: Several companies have done initial evaluation of automated mud control with limited success. one company, Halliburton, has an automated system but has had little success so far. A determination of the economics of the mud industry and how mud automation effects rig owners is necessary.

Application: Because of estimated costs, this concept will primarily apply to large drilling rigs. 
TABLE 4-6

PROJECT 2-5, MUD AUTOMATION SYSTEMS

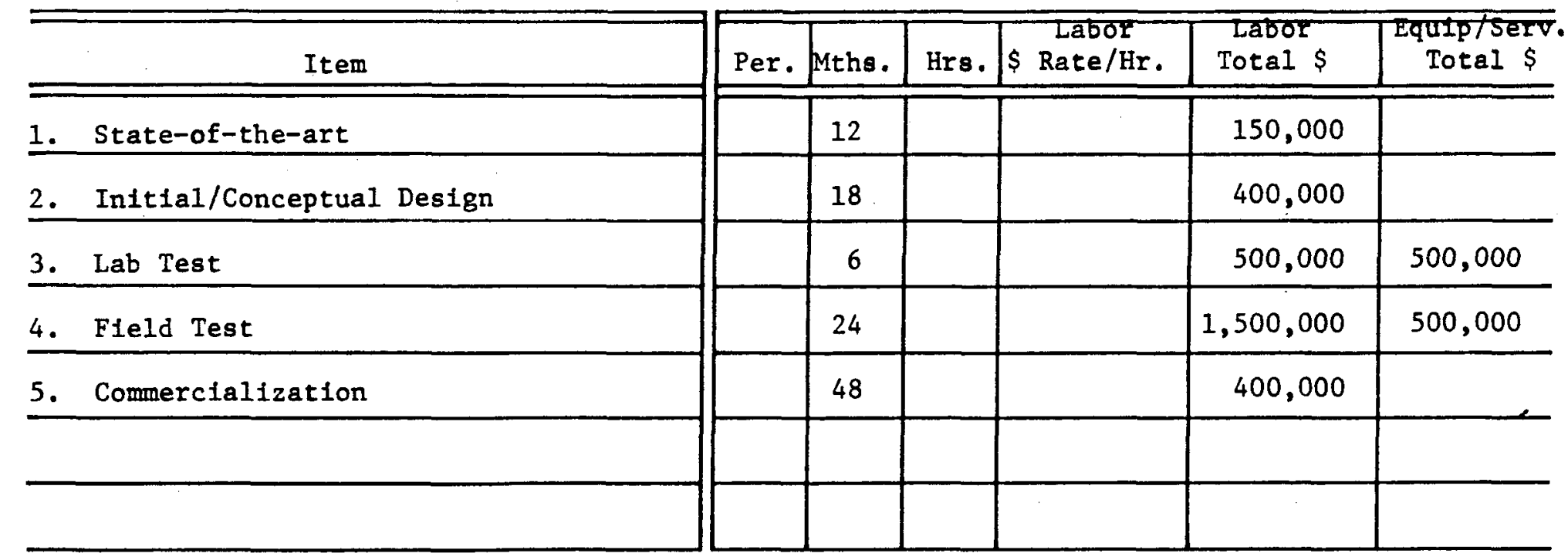


Project 3-1 - Rig Instrumentation/Sensing Equipment (Table 4-7)

Description: The application of automation-computerization techniques is dependent upon the ability of equipment to sense drilling conditions or movement of components. Unique problems encountered are not solvable by use of conventional. techniques. Project 4-1 will evaluate instrumentation and sensing problems, determine solutions, and assist in developing, testing, and advancing rig instrumentation and sensing equipment techniques. Rugged, inexpensive equipment with a reasonable degree of accuracy is essential.

State of the Art: Several large companies have designed and are marketing sensing equipment. Devices now on the market are adequate for normal drilling operations, but will be inadequate for advanced applications.

Application: Industry acceptance of rig automationcomputerization will require the use of instrumentation and sensing equipment. Since proper instrumentation is fundamertal to optimization/computer programs and telemetry programs, project $3-1$ should begin immediately and should receive top priority. 


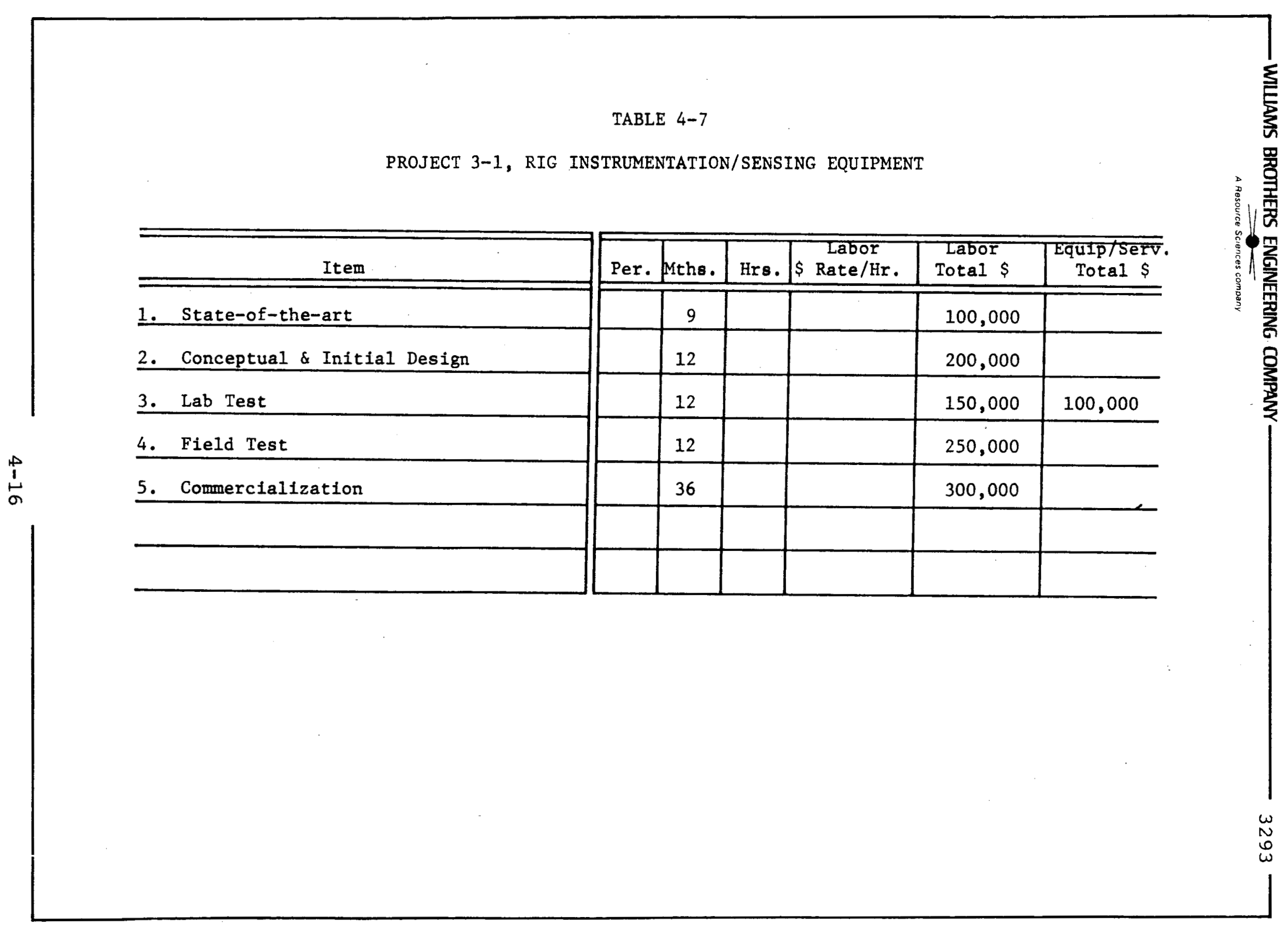




\section{Project 3-2 - Rig Floor Data Display/Analysis Equipment (Table 4-8)}

Description: The basic drilling operation includes the monitoring and varying of a number of operating conditions. Monitoring these conditions from a central location on the rig floor with a visual readout system incorporating max-min operating limits will enhance the operation. Proper application of this type of equipment will be the first step in the computerization of the drilling operation for all segments of the drilling industry. Equipment can be combined with home office computer systems. Solution to training problems in equipment use is essential. This project will evaluate available equipment, define equipment needed, and assist in the development, testing, and application of devices.

State of the Art: Page and Tri-state may have the most advanced equipment. Large, expensive systems are being used for onshore and offshore rigs where daily operational costs are adequate to justify such equipment. Equipment available for use on small rigs is sparse.' Equipment is new and has not been properly field tested to determine its full capability.

Application: Since Data Display and Analysis Equipment will be applicable to the general drilling industry, Project 3-2 should be given priority. 
TABLE 4-8

PROJECT 3-2, RIG FLOOR DATA DISPLAY/ANALYSIS EQUIPMENT

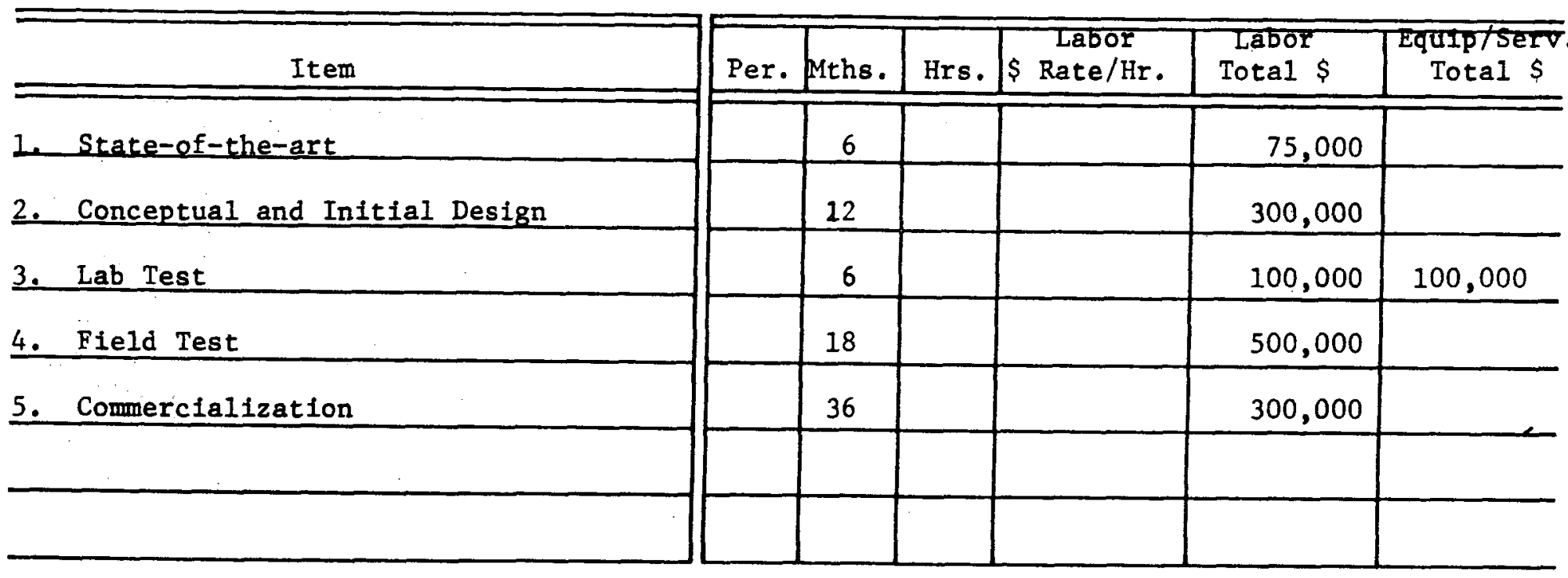


Project 3-3 - Down-Hole Logging - Telemetry System (Table 4-9)

Description: DOE participated in the field testing of the TELECO mud-pulse telemetry system, a method for transmitting down-hole information through a flowing column of drilling mud. Continued field testing is required to determine the full capability of the system.

State of the Art: Numerous companies are attempting to transmit down-hole data to the surface. TELECO appears to be one of the best systems. Information and reports available from DOE provide data to start an effective field test program. Project $3-3$ is a continuation of DOE programs.

Application: This technology, due to the probable expenses involved, will apply primarily to deep hole drilling. 
TABLE 4-9

PROJECT 3-3, DOWN-HOLE LOGGING - TELEMETRY SYSTEM

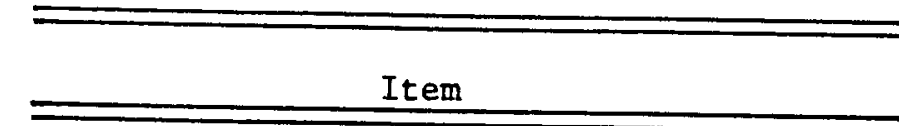

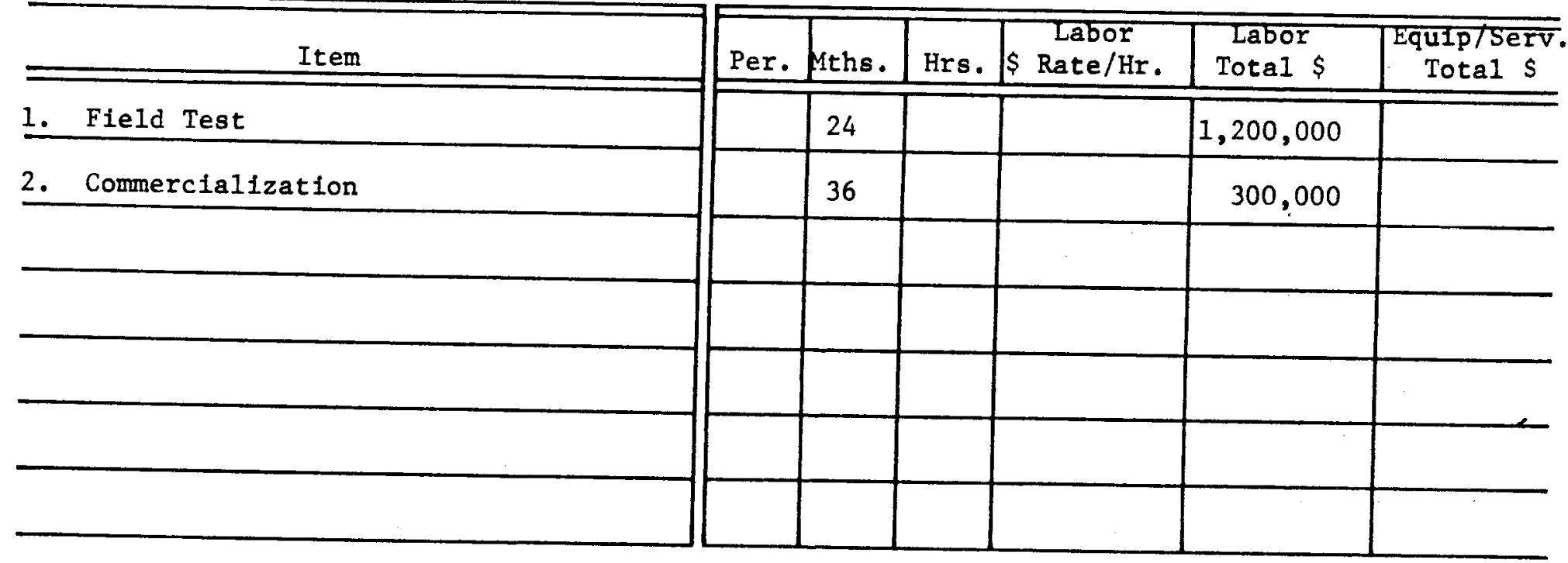


Project 3-4 - Surface Logging - Telemetry System (Table 4-10)

Description: During the drilling operation, optimization and correction of drilling equipment control variables is dependent upon the geological strata and down-hole drilling equipment conditions. Effort is being made to monitor and transmit information from the bottom of the hole to the drilling floor. Because this process is expensive, it is limited to deep hole, platform, arctic, and offshore drilling. An inexpensive surface monitoring system that would perform preliminary tests on rock chips taken from the shale shaker will be considered. Information from these tests, combined with rig floor operating data, could predict down-hole conditions. Although the results would be less accurate than down-hole systems, the process would be less expensive and thus applicable to a larger number of rigs. Project 3-4 will evaluate existing systems, determine economic feasibility, and assist in system development.

State of the Art: The versions of this concept currently available to industry are excessively costly and therefore limited in use. Although Mobil and Conoco believe surface logging-telemetry systems have possibilities, Project 3-4 will be on a go/no-go basis.

Application: Based on the economics involved and the adequacy of the information obtained, this technology could apply to all segments of the industry. 
TABLE 4-10

PROJECT 3-4, SURFACE LOGGING - TELEMETRY SYSTEMS

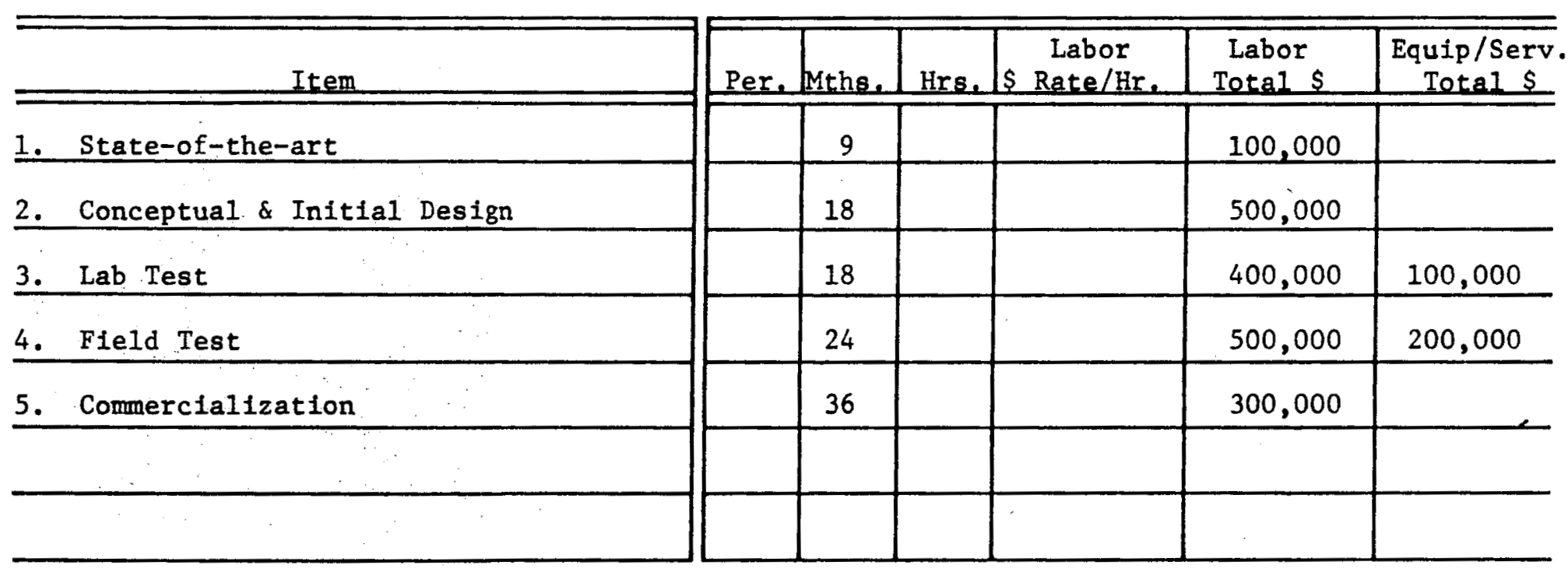




\section{Project 3-5 - Computer Controlled Drilling (Table 4-11)}

Description: Computer controlled drilling consists of maximizing the drilling variables to increase penetration rate of the drilling process. Considerable effort has been expended by industry to define and field test this concept.

Project 3-5 will evaluate existing computer controlled drilling systems, assist in their improvement, and foster their use by the drilling industry. System design will use high reliability, rugged hardware and be set up for use on all rig operations.

State of the Art: Amoco is the leader in computer controlled drilling technology and claims considerable success. Other corporations have also evaluated this technology, but unfortunately, use of computer controlled drilling technology is limited.

Application: Computer controlled drilling technology can be used by all segments of the industry. System design will emphasize commercialization. 
TABLE 4-11

PROJECT 3-5, COMPUTER CONTROLLED DRILLING

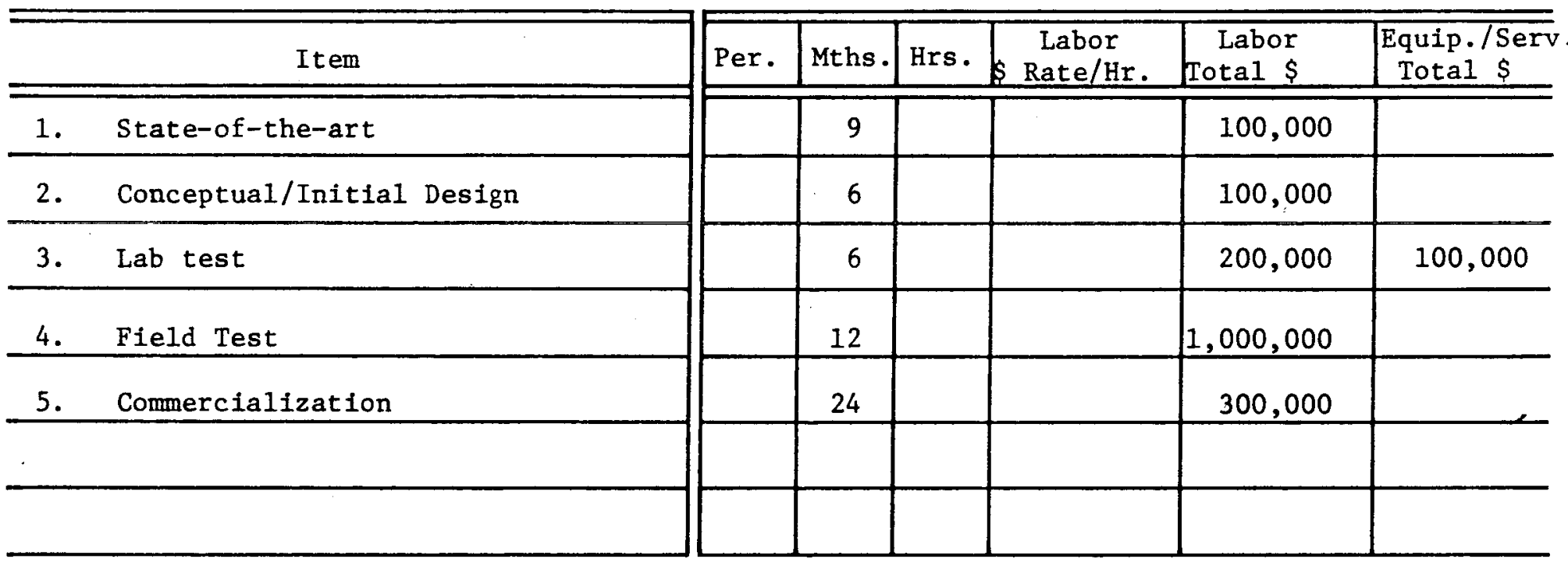


Project 4-1 - Jet Erosion Drilling (Table 4-12)

Description: Jet erosion drilling uses high pressure mud jets to fracture and break the rock at the bottom of the hole. Industry has experimentally evaluated and field tested this technology and results have been mixed. However, work remains to be done.

Project 4-1 will evaluate the work done and help expand jet erosion drilling technology to facilitate building a complete system which can be made commercially available. Lab work accomplished prior to field testing will avoid field costs.

State of the Art: Exxon, Gulf, and shell have worked on versions of this concept and have obtained encouraging results. Data will be evaluated to determine proper application.

Application: Cost will limit use of jet erosion drilling to deep land, platform, and arctic drilling. 


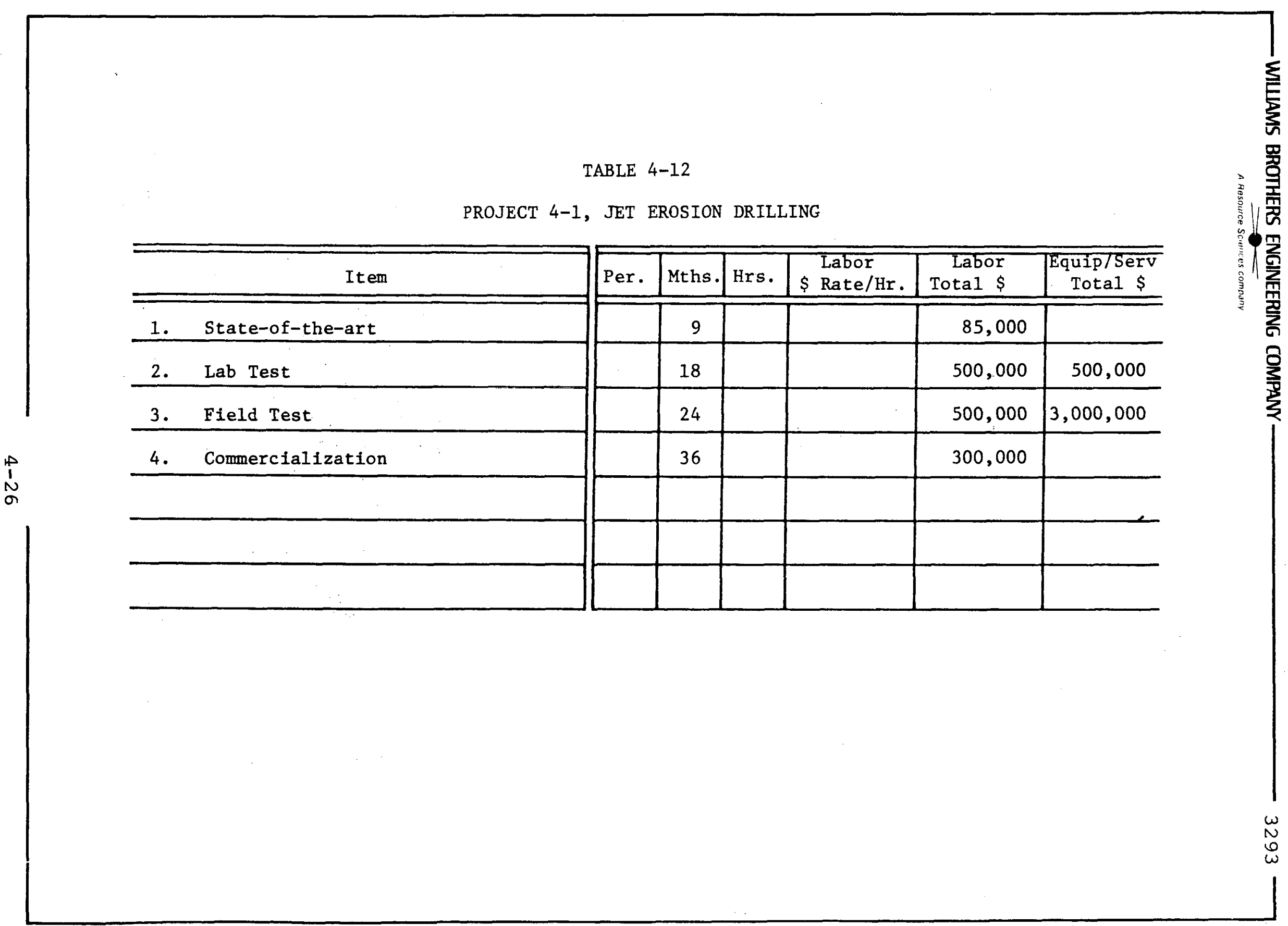




\section{Project 5-1 - Down-Hole Motor Technology (Table 4-13)}

Description: Down-hole motor technology utilizes a down-hole motor to impart rotating force to the drilling bit. DOE has sponsored projects participating in the development of down-hole motor technology. Project 5-1 will help expand this technology and make it available to the industry.

State of the Art: Project 5-1 is a continuation of DOE programs. Numerous companies and government groups are working on phases of down-holw motor technology.

Application: Cost will limit application of down-hole motor technology to deep land, platform, and arctic drilling. 
PROJECT 5-1, DOWN-HOLE MOTOR TECHNOLOGY

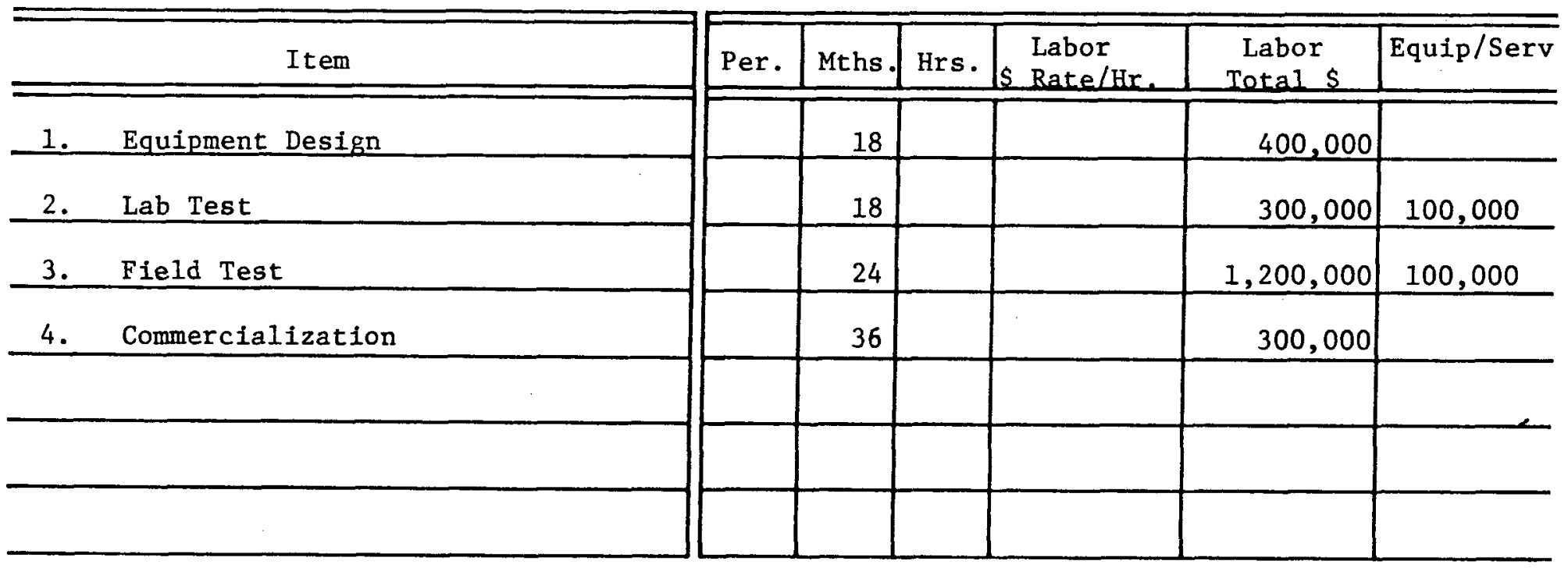


Project 6-1 - stratapax and Bit Technology (Table 4-14)

Description: Project 6-1, a continuation of DOE programs, will investigate, analyze, and field test bonding techniques and the technologies used to develop new and advanced drilling bits.

DOE has participated in the development of stratapax and bit technology. Continuation and expansion of project 6-1 will help advance the drilling industry.

State of the Art: Sandia Labs has developed techniques necessary for attaching man-made diamonds to drilling bit bodies.

Application: Stratapax and bit technology can be applied to all areas of the drilling industry. 


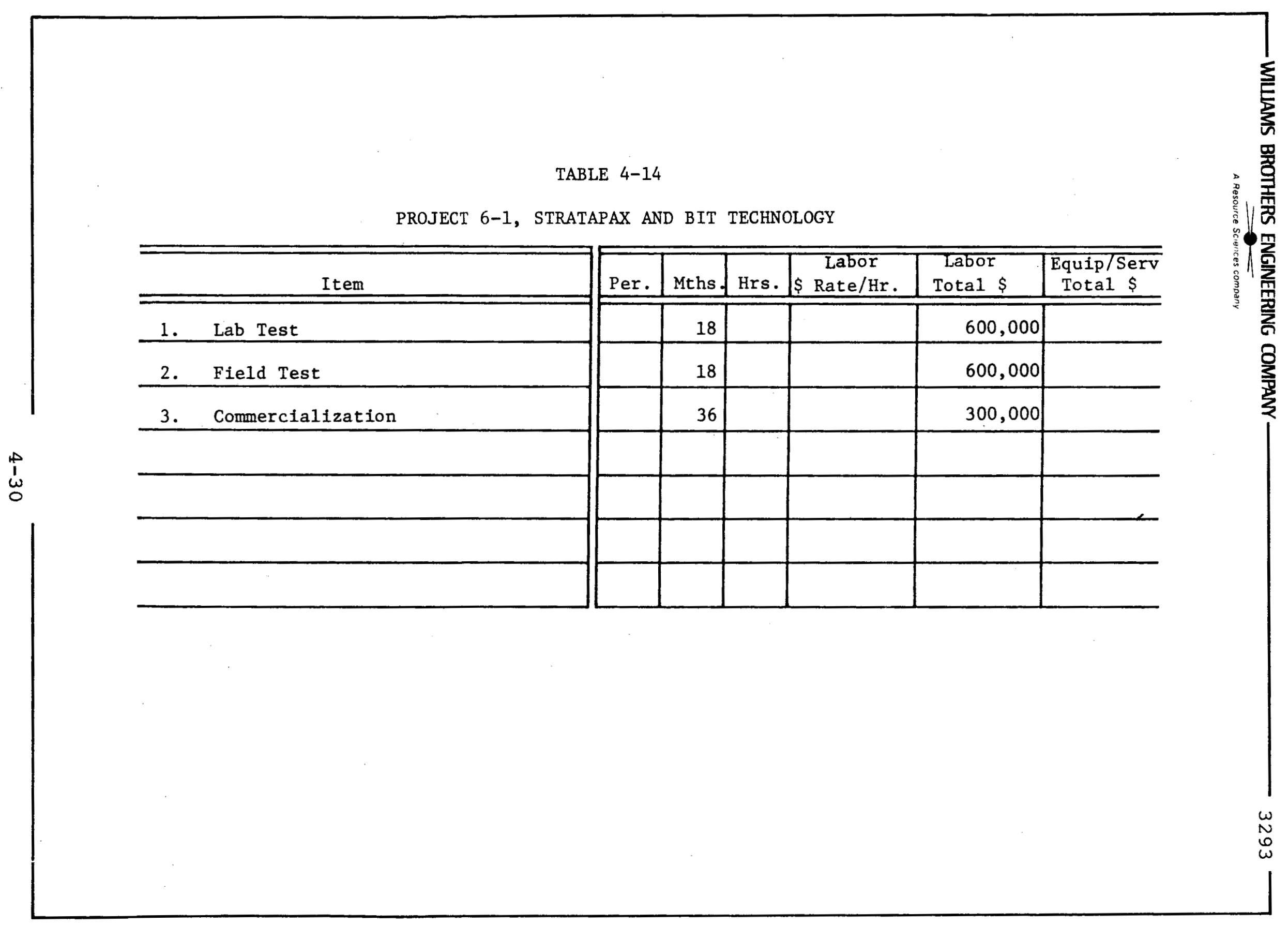


Project 7-1 - Blowout Preventer Equipment (Table 4-15)

Description: Blowout Preventors (BOP) control unstable down-hole well conditions. Their use is mandatory to the safety of the drilling operation.

Project 7-1 will evaluate BOP technology, define areas requiring improvement, and assist industry in advancing BOP technology. Potential improvement may be BOP automation and down-hole BOP systems. Project 7-1 would be undertaken on a go/no go basis.

State of the Art: Industry has advanced BOP technology to meet the needs and economies of the drilling industry.

However, little has been done to accelerate BOP automation technology. Analysis to determine project potential is necessary. BOP automation may be applicable only for offshore or dangerous areas.

Application: BOP technology applies to all segments of the drilling industry. 
Project 8-1 - Advanced Drilling Equipment (Table 4-16)

Description: Project 8-1 will define, evaluate, and if necessary, assist in the development of advanced drilling concepts. These concepts could range from advanced offshore riser systems to advanced land mobil rig designs. Project 8-1 will provide incentive to accelerate industry advancement by defining the scope and economic value of new concepts and procedures. It will require a go/no go basis.

State of the Art: Industry will provide new technology essential to its economic need. Little effort is made to advance the industry over and above that which is absolutely essential.

Application: Project 8-1 can apply to all phases of the drilling industry and is designed to encourage advanced thinking and development. 


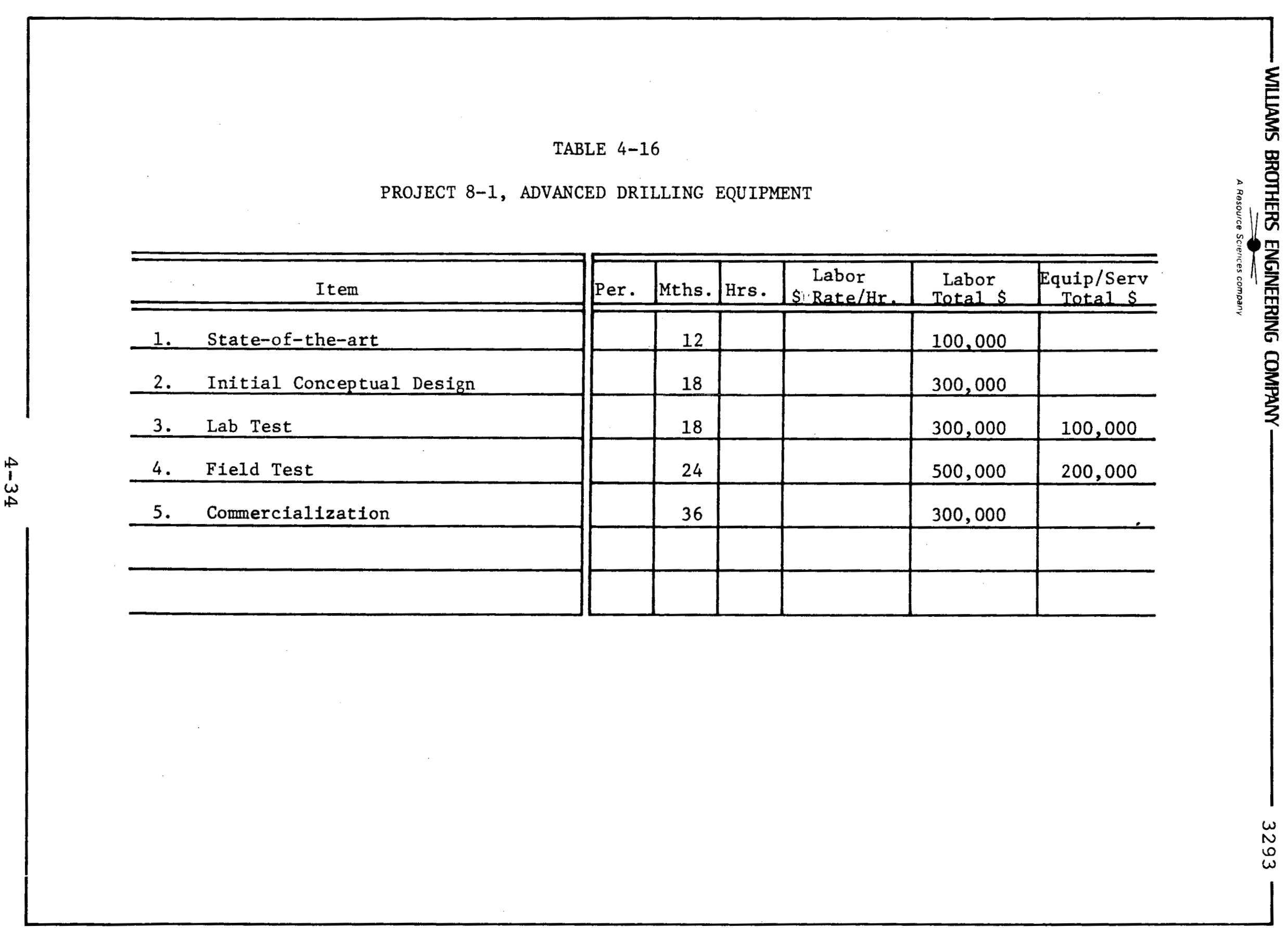




\section{SECTION 5}

PROJECT BENEFIT ANALYSIS

In the drilling equipment industry, a new product is ready to market only after extensive field testing proves it will improve productivity and/or efficiency. To measure productivity and efficiency, drilling penetration rate was selected as a standard for comparison. Since drilling penetration rate measures the ability of a drilling rig to make a hole, rig productivity increases if this ability can be improved.

Efficiency in the drilling operation includes engine maintenance, tripping operations, logistics, etc. support operations, combined with the drilling operation, produce a hole. Therefore, if the efficiency of support operations can be improved, the overall ability of a rig to produce a hole can be improved, and the rig's productivity increases.

To determine drilling penetration rate increase of each project the following analysis was made:

\section{Evaluate Project vs. Rig Depth Capability}

As the projects involved are economically viable only on certain sizes (or depths) or rigs, a review was made of each project to determine its rig size applicability (Table 5-1). This determination was based on a literature survey, industry economic studies, and discussion with key industry representatives. 
2. Projected Size of Rig Market and Rig Size Distribution to 1995

To determine future product sales potential, an analysis of the rig market by rig size and total rig fleet was made to the year 1995. Rig size was determined by reviewing the rig size distribution for the last eight years (Table 5-2). Although this analysis shows certain percentage variations a degree of judgment was used when extrapolating this data to the year 1995 (Table 5-3).

\section{Estimated Drilling Preparation Rate Improvement}

An estimate of drilling penetration rate improvement was made for each project based on the limited field or laboratory information available on similar projects (Table 5-4).

\section{Project Potential}

To define the meaning in terms of overall drilling penetration rate improvement to the industry in general, for each of the projects, the information attained in Steps 1, 2 and 3 above was compiled in Tables 5-5 to 5-20. In each table, total estimated rig count from Table 5-3 was listed. Estimated applicable rig count for each year was determined by multiplying the percentage of rigs (Table 5-1 as it applies to Table 5-3) by the estimated rig count of Table 5-3.

Estimated rigs using the system is a judgment factor of market drilling penetration rate multiplied by estimated number of applicable rigs. 
Estimated system utilization rate is a judgment factor based on a literature survey and discussion with representatives of the industry.

Estimated maximum penetration rate is taken from Table 5-4.

Penetration rate increase was obtained by multiplying the number of estimated applicable rigs, by the number of estimated rigs using the system, by the estimated system utilization rate, and by the maximum drillingpenetration rate increase. 
TABLE 5-1

PROJECT - RIG DEPTH APPLICABILITY

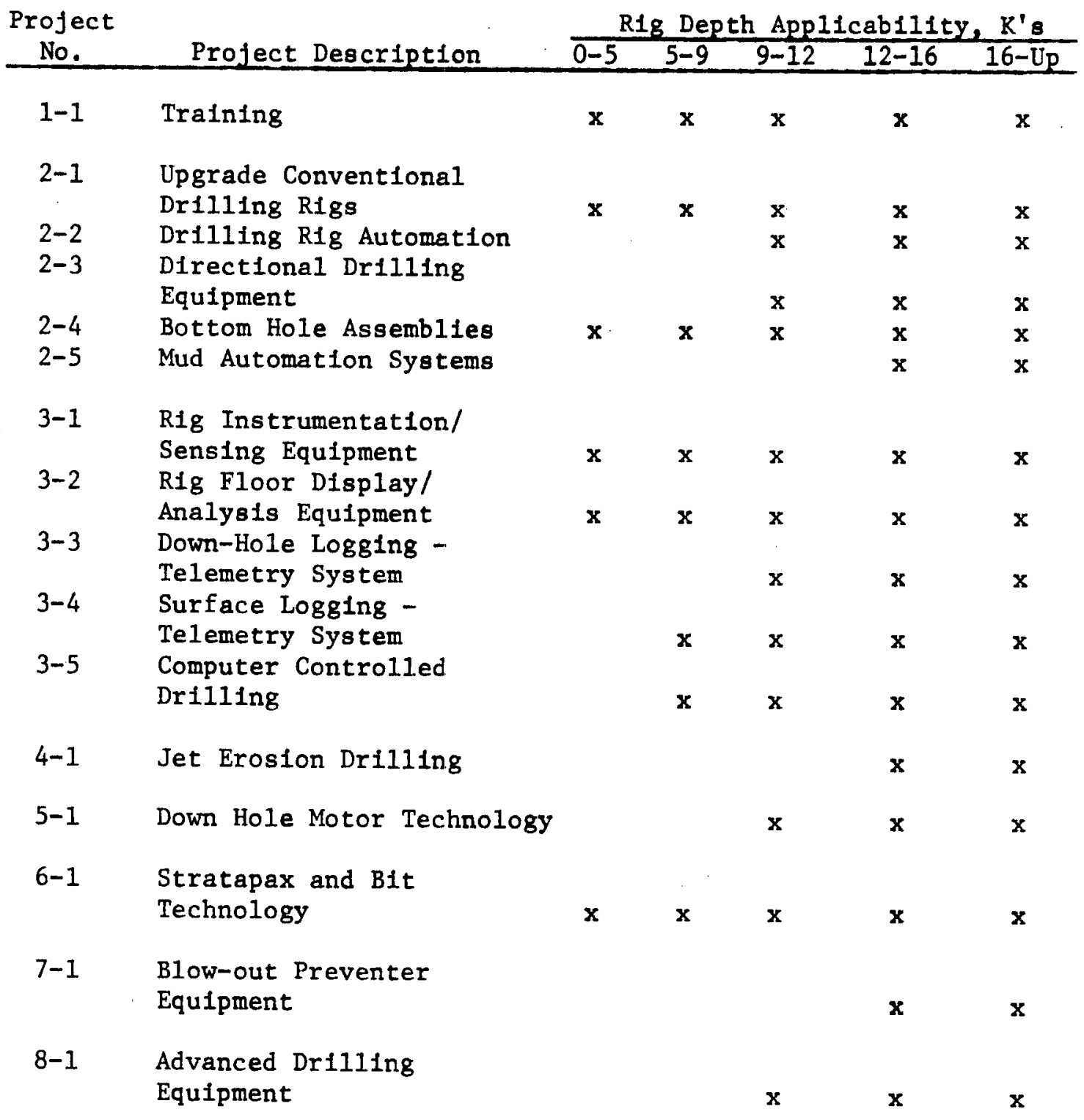


TABLE 5-2

HISTORICAL U.S. ROTARY RIG DEPTH DISTRIBUTION (Percent of Total Rig Count)

\begin{tabular}{|c|c|c|c|c|c|}
\hline \multirow{2}{*}{ Year } & \multicolumn{5}{|c|}{ Rig Size, $K^{\prime}$ s } \\
\cline { 2 - 6 } 1970 & $0-5$ & $5-9$ & $9-12$ & $12-16$ & $16-$ Up \\
\hline 1971 & 23 & 31 & 17 & 11 & 18 \\
1972 & 23 & 29 & 18 & 11 & 19 \\
1973 & 20 & 29 & 17 & 9 & 22 \\
1974 & 20 & 28 & 18 & 11 & 24 \\
1975 & 19 & 29 & 19 & 11 & 22 \\
1970 & 21 & 29 & 17 & 11 & 22 \\
1977 & 22 & 25 & 19 & 11 & 23 \\
\hline
\end{tabular}

Notes :

1. Rig size denotes drilling depth capability in thousands of feet.

2. Data includes both land and offshore rigs.

3. Source: Drilling DCW, Annual Rotary Rig Census. 
TABLE 5-3

PROJECTED U.S. ROTARY RIG DEPTH DISTRIBUTION (Percent of Total Rig Count)

\begin{tabular}{|c|c|c|c|c|c|c|}
\hline \multirow[b]{2}{*}{ Year } & \multicolumn{5}{|c|}{ Rig Size, $K^{\prime} s$} & \multirow{2}{*}{$\begin{array}{l}\text { Total } \\
\text { Rigs }\end{array}$} \\
\hline & $0-5$ & $5-9$ & $9-12$ & $12-16$ & $16-U_{p}$ & \\
\hline 1980 & 21 & " 20 & 23 & 12 & 24 & 2570 \\
\hline 1981 & 20 & 19 & 24 & 13 & 24 & 2750 \\
\hline 1982 & 19 & 18 & 24 & 14 & 25 & 2910 \\
\hline 1983 & 18 & 17 & 25 & 15 & 25 & 3060 \\
\hline 1984 & 18 & 16 & 25 & 15 & 26 & 3200 \\
\hline 1985 & 17 & 16 & 26 & 15 & 26 & 3340 \\
\hline 1986 & 17 & 15 & 26 & 16 & 26 & 3470 \\
\hline 1987 & 16 & 15 & 27 & 16 & 26 & 3600 \\
\hline 1988 & 16 & 14 & 27 & 17 & 26 & 3730 \\
\hline 1989 & 15 & 14 & 28 & 17 & 26 & 3860 \\
\hline 1990 & 15 & 13 & 28 & 17 & 27 & 4000 \\
\hline 1991 & 14 & 13 & 29 & 17 & 27 & 4140 \\
\hline 1992 & 14 & 12 & 29 & 18 & 27 & 4280 \\
\hline 1993 & 14 & 11 & 30 & 18 & 27 & 4420 \\
\hline 1994 & 13 & 11 & 31 & 18 & 27 & 4560 \\
\hline 1995 & 13 & 11 & 31 & 18 & 27 & 4700 \\
\hline
\end{tabular}

Notes: 1. Rig size denotes drilling depth capability in thousands of feet.

2. Data includes both land and offshore rigs. 
TABLE 5-5

PROJECT 1-1, POTENTIAL

YEARLY PENETRATION RATE INCREASE (PERCENT)

\begin{tabular}{|c|c|c|c|c|c|c|c|c|}
\hline \multirow[b]{2}{*}{ Year } & \multirow[b]{2}{*}{$\begin{array}{c}\text { Estimated } \\
\text { Rig Count } \\
\text { Total }\end{array}$} & \multicolumn{2}{|c|}{$\begin{array}{l}\text { Estimated } \\
\text { Applicable Rigs }\end{array}$} & \multicolumn{2}{|c|}{$\begin{array}{c}\text { Estimated } \\
\text { Rigs Using System }\end{array}$} & \multirow{2}{*}{$\begin{array}{c}\text { Estimated } \\
\text { System } \\
\text { Utilization } \\
\text { Rate } \\
\%\end{array}$} & \multirow{2}{*}{$\begin{array}{c}\text { Estimated } \\
\text { Maximum } \\
\text { Penctration } \\
\text { Rate } \\
\text { Incrcase } \\
\% \\
\end{array}$} & \multirow{2}{*}{$\begin{array}{c}\text { Penetration } \\
\text { Rate } \\
\text { Increase of } \\
\text { Total Rig } \\
\text { Count } \\
\%\end{array}$} \\
\hline & & $\begin{array}{c}\text { Rig } \\
\text { Courit } \\
\%\end{array}$ & No. & $\begin{array}{c}\text { Rig } \\
\text { Count } \\
\% \\
\end{array}$ & No. & & & \\
\hline 1980 & 2570 & 100 & 2570 & 5 & 129 & 75 & 20 & .8 \\
\hline 1981 & 2750 & 100 & 2750 & 10 & 275 & 75 & 20 & 1. 5 \\
\hline 1982 & 2910 & 100 & 2910 & 15 & 436 & 75 & 20 & 2. 3 \\
\hline 1983 & 3060 & 100 & 3060 & 25 & 765 & 75 & 20 & 3.8 \\
\hline 1984 & 3200 & 100 & 3200 & 35 & 1120 & 75 & 20 & 5.3 \\
\hline 1985 & 3340 & 100 & $33+0$ & 45 & 1503 & 75 & 20 & 6.8 \\
\hline 1986 & 3470 & 100 & 3.470 & 55 & 1909 & 75 & 20 & 8. 3 \\
\hline 1987 & 3600 & 100 & 3600 & 65 & 2.340 & 75 & 20 & 9.8 \\
\hline 1988 & 3730 & 100 & 3730 & 70 & 26,11 & 75 & 20 & 10.5 \\
\hline 1989 & 3860 & 100 & 3860 & 75 & <8y5 & 75 & 20 & 11.2 \\
\hline 1990 & 4000 & 100 & 4000 & 75 & 3000 & 75 & 20 & 11.2 \\
\hline 1991 & 4140 & 100 & -1140 & 75 & 3105 & 75 & 20 & 11.2 \\
\hline 1992 & +280 & 100 & 4280 & 75 & 3210 & 75 & 20 & 11.2 \\
\hline 1993 & 4420 & 100 & $4+20$ & 75 & 3315 & 75 & 20 & 11.2 \\
\hline 1994 & 4560 & 100 & 456,0 & 75 & $3+20$ & 75 & 20 & 11.2 \\
\hline 1905 & 4700 & 100 & 4700 & 75 & 3525 & 75 & 20 & 11.2 \\
\hline
\end{tabular}

Estimated Rig Size Applicable: All Rigs

Estimated Maximum System Utilization Rate: $75 \%$

Estimated Maximum Penetration Rate Increase: $20 \%$ 


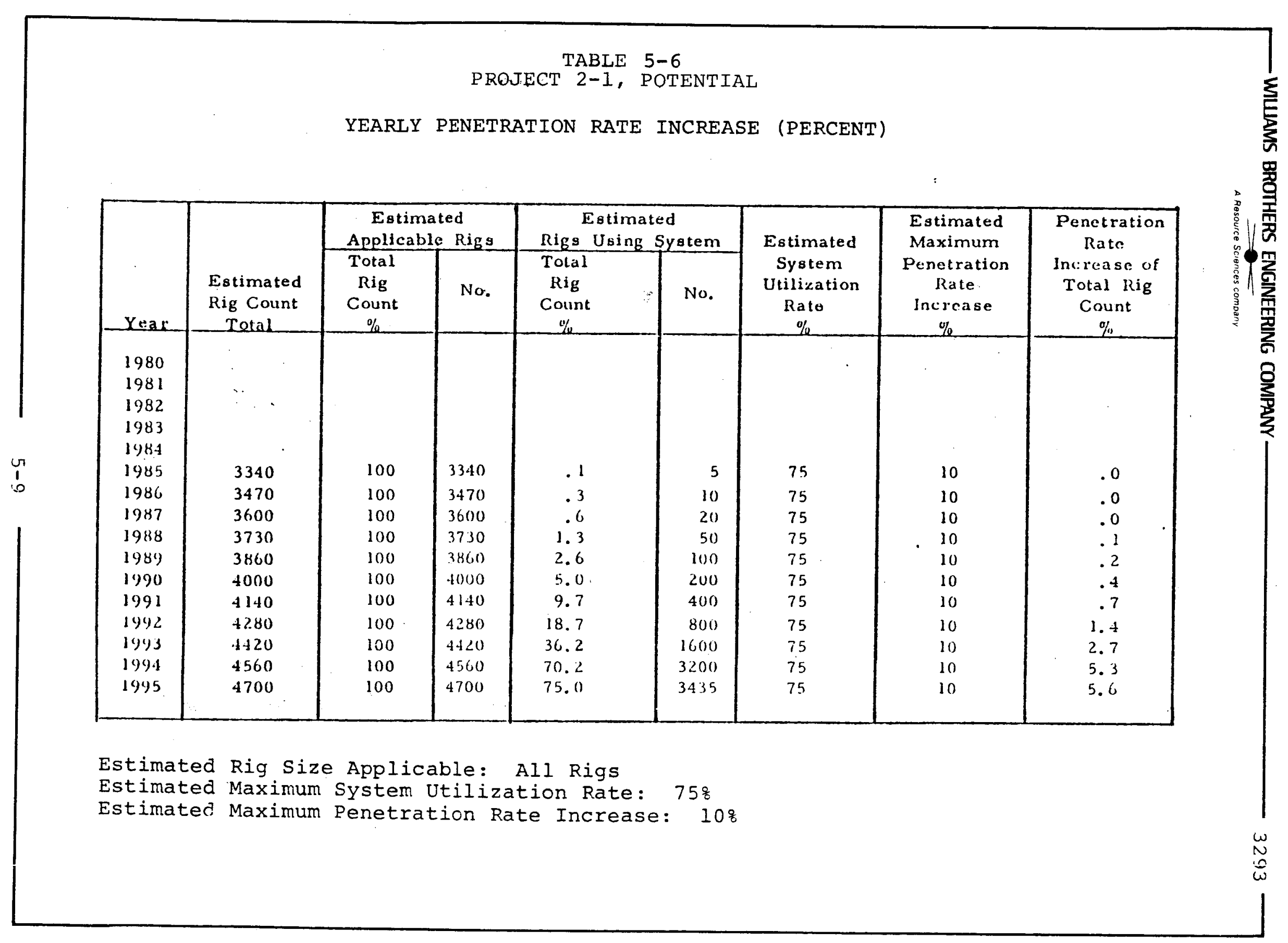



5-9

PROJECT 2-4, POTENTIAL

YEARLY PENETRATION RATE INCREASE (PERCENT)

\begin{tabular}{|c|c|c|c|c|c|c|c|c|}
\hline \multirow[b]{2}{*}{ Year } & \multirow[b]{2}{*}{$\begin{array}{c}\text { Estimated } \\
\text { Rig Count } \\
\text { Total }\end{array}$} & \multicolumn{2}{|c|}{$\begin{array}{c}\text { Estimated } \\
\text { Applicable Rigs }\end{array}$} & \multicolumn{2}{|c|}{$\begin{array}{c}\text { Estimaled } \\
\text { Rigs Using System }\end{array}$} & \multirow{2}{*}{$\begin{array}{c}\text { Estimated } \\
\text { System } \\
\text { Utilization } \\
\text { Rate } \\
\% \\
\end{array}$} & \multirow{2}{*}{$\begin{array}{c}\text { Estimated } \\
\text { Maxinum } \\
\text { Penetration } \\
\text { Rate } \\
\text { Increase } \\
\%\end{array}$} & \multirow{2}{*}{$\begin{array}{c}\text { Penetration } \\
\text { Rate } \\
\text { Increase of } \\
\text { Total llig } \\
\text { Count } \\
\%\end{array}$} \\
\hline & & $\begin{array}{c}\text { Rig } \\
\text { Count } \\
\%\end{array}$ & No. & $\begin{array}{c}\text { Rig } \\
\text { Count } \\
\%\end{array}$ & No. & & & \\
\hline $\begin{array}{l}1980 \\
1981\end{array}$ & & & & & & & & \\
\hline 1982 & 2910 & 100 & 2910 & .2 & 5 & 75 & 20 & .0 \\
\hline 1983 & 3060 & 100 & 3060 & .3 & 10 & 75 & 20 & .0 \\
\hline 1984 & 3200 & 100 & 3200 & .9 & 30 & 75 & 20 & .1 \\
\hline 1985 & 3340 & 100 & 3340 & 2. 1 & 70 & 75 & 20 & .3 \\
\hline 1986 & 3470 & 100 & 3470 & 4.3 & 150 & 75 & 20 & .6 \\
\hline 1987 & 36,00 & 100 & 3600 & 8.3 & 300 & 75 & 20 & 1.2 \\
\hline 1988 & 3730 & 100 & 3730 & 16.1 & 600 & 75 &.$\quad 20$ & 2.4 \\
\hline 1989 & 3860 & 100 & $3 \$ 6,0$ & 31.1 & 1200 & 75 & 20 & 4.7 \\
\hline 1990 & 4000 & 100 & 4000 & 60.0 & 2400 & 75 & 2.0 & 9.0 \\
\hline 1991 & 4140 & 100 & 4140 & 75.0 & 3105 & 75 & 20 & 11.2 \\
\hline 1992 & 4280 & 100 & 4280 & 75.0 & 3210 & 75 & 20 & - 11.2 \\
\hline 1993 & $4+20$ & 100 & 4420 & 75.0 & 3315 & 75 & 20 & 11.2 \\
\hline 1994 & 4560 & 100 & 4560 & 75.0 & 3420 & 75 & 20 & 11.2 \\
\hline 1495 & 4700 & 100 & 4700 & 75.0 & 3525 & 75 & 20 & 11.2 \\
\hline
\end{tabular}

Estimated Rig Size Applicable: All Rigs

Estimated Maximum System Utilization Rate: $75 \%$

Estimated Maximum Penetration Rate Increase: $20 \%$ 
TABLE 5-10

PROJECT 2-5, POTENTIAL

YEARLY PENETRATION RATE INCREASE (PERCENT).

\begin{tabular}{|c|c|c|c|c|c|c|c|c|}
\hline \multirow[b]{2}{*}{ Yeax } & \multirow[b]{2}{*}{$\begin{array}{c}\text { Estimated } \\
\text { Rig Count } \\
\text { Iotal }\end{array}$} & \multicolumn{2}{|c|}{$\begin{array}{c}\text { Estimated } \\
\text { Applicable Riga }\end{array}$} & \multicolumn{2}{|c|}{$\begin{array}{c}\text { Estimated } \\
\text { Rigs Using System }\end{array}$} & \multirow{2}{*}{$\begin{array}{c}\text { Estimated } \\
\text { System } \\
\text { Utilization } \\
\text { Rate } \\
\% \\
\end{array}$} & \multirow{2}{*}{$\begin{array}{c}\text { Estimated } \\
\text { Maximum } \\
\text { Penetration } \\
\text { Rate } \\
\text { Increase } \\
\text { de } \\
\end{array}$} & \multirow{2}{*}{$\begin{array}{c}\text { Penctration } \\
\text { Rate } \\
\text { Increase of } \\
\text { Total Rig } \\
\text { Count } \\
\% \\
\end{array}$} \\
\hline & & $\begin{array}{c}\text { Total } \\
\text { Rig } \\
\text { Count } \\
\% \\
\end{array}$ & No. & $\begin{array}{c}\text { Total } \\
\text { Rig } \\
\text { Count } \\
\%\end{array}$ & No. & & & \\
\hline \\
\hline 1981 & & & & & & & & \\
\hline 1982 & & & & & & & & \\
\hline 1983 & & & & & & & 8 & \\
\hline 1934 & & & & & & & & \\
\hline 1985 & & & & & & & & \\
\hline 1986 & & & & & & & & \\
\hline 1987 & 3600 & 42 & 1512 & .1 & 3 & 25 & 5 & .0 \\
\hline 1988 & 3730 & 43 & 1604 & .3 & 10 & 25 & 5 & .0 \\
\hline 1989 & 3860 & 43 & 1660 & .5 & 20 & 25 & 5 & .0 \\
\hline 1990 & 4000 & 44 & 1760 & 1.2 & 50 & 25 & 5 & .0 \\
\hline 1991 & 4140 & 44 & 1822 & 1.9 & 80 & 25 & 5 & .0 \\
\hline 1992 & 4280 & 45 & 1926 & 2.8 & 120 & 25 & 5 & .0 \\
\hline 1993 & 4420 & 45 & 1989 & 5.7 & 250 & 25 & 5 & .1 \\
\hline 1994 & 4560 & 45 & 2052 & 8.8 & 400 & 25 & 5 & .1 \\
\hline 1995 & 4700 & 45 & 2115 & 11.3 & 530 & 25 & 5 & .1 \\
\hline
\end{tabular}

Estimated Rig Size Applicable: $12-16^{\mathrm{K}}, 16^{\mathrm{K}}$-Up

Estimated Maximum System Utilization Rate: $25 \%$

Estimated Maximum Penetration Rate Increase: 5\% 
TABLE 5-1I

PROJECT 3-1, POTENTIAL

YEARLY PENEIRATION RATE INCREASE (PERCENT)

\begin{tabular}{|c|c|c|c|c|c|c|c|c|}
\hline \multirow[b]{2}{*}{ Year } & \multirow[b]{2}{*}{$\begin{array}{l}\text { Estimated } \\
\text { Rig Count } \\
\text { Tetal }\end{array}$} & \multicolumn{2}{|c|}{$\begin{array}{l}\text { Estimated } \\
\text { Applicable Rigs }\end{array}$} & \multicolumn{2}{|c|}{$\begin{array}{c}\text { Estimated } \\
\text { Rigs Using System }\end{array}$} & \multirow{2}{*}{$\begin{array}{c}\text { Eutimated } \\
\text { System } \\
\text { Utilization } \\
\text { Rate } \\
\%\end{array}$} & \multirow{2}{*}{$\begin{array}{c}\text { Estimated } \\
\text { Maximum } \\
\text { Penetration } \\
\text { Rate } \\
\text { Increase } \\
\% \\
\end{array}$} & \multirow{2}{*}{$\begin{array}{c}\text { Penetration } \\
\text { Rate } \\
\text { Increase of } \\
\text { Total Rig } \\
\text { Count. } \\
\% \\
\end{array}$} \\
\hline & & $\begin{array}{c}\text { Rig } \\
\text { Count } \\
\%\end{array}$ & No. & $\begin{array}{c}\text { Rig } \\
\text { Count } \\
\% \\
\end{array}$ & No. & & & \\
\hline 1980 & 2570 & & & & & & & \\
\hline 1981 & 2750 & & & & & & & \\
\hline 1982 & 2910 & & & & & & & \\
\hline 1983 & 3060 & & & & & & & \\
\hline 1984 & 3200 & & & & & & & \\
\hline 1985 & 3340 & & & & & & & \\
\hline 1986 & 3470 & & & & & & & \\
\hline 1987 & 3600 & & & & & & & \\
\hline 1988 & 3730 & & & & & & & \\
\hline 1969 & 3860 & & & & & & - & \\
\hline 1990 & 4000 & & & & & & & \\
\hline 1991 & $41+0$ & & & & & & & \\
\hline 1992 & 4280 & & & & & & & \\
\hline 1993 & 4420 & & & & & .. & & \\
\hline 1994 & 4560 & & & & & & & \\
\hline 1995 & 4700 & & & & & & & \\
\hline
\end{tabular}

Estimated Rig Size Applicable: All Rigs

Estimated Maximum System Utilization Rate:

Estimated Maximum Penetration Rate Increase: -- 
TABLE 5-12

PROJECT 3-2, POTENTIAL

YEARLY PENETRATION RATE INCREASE (PERCENT)

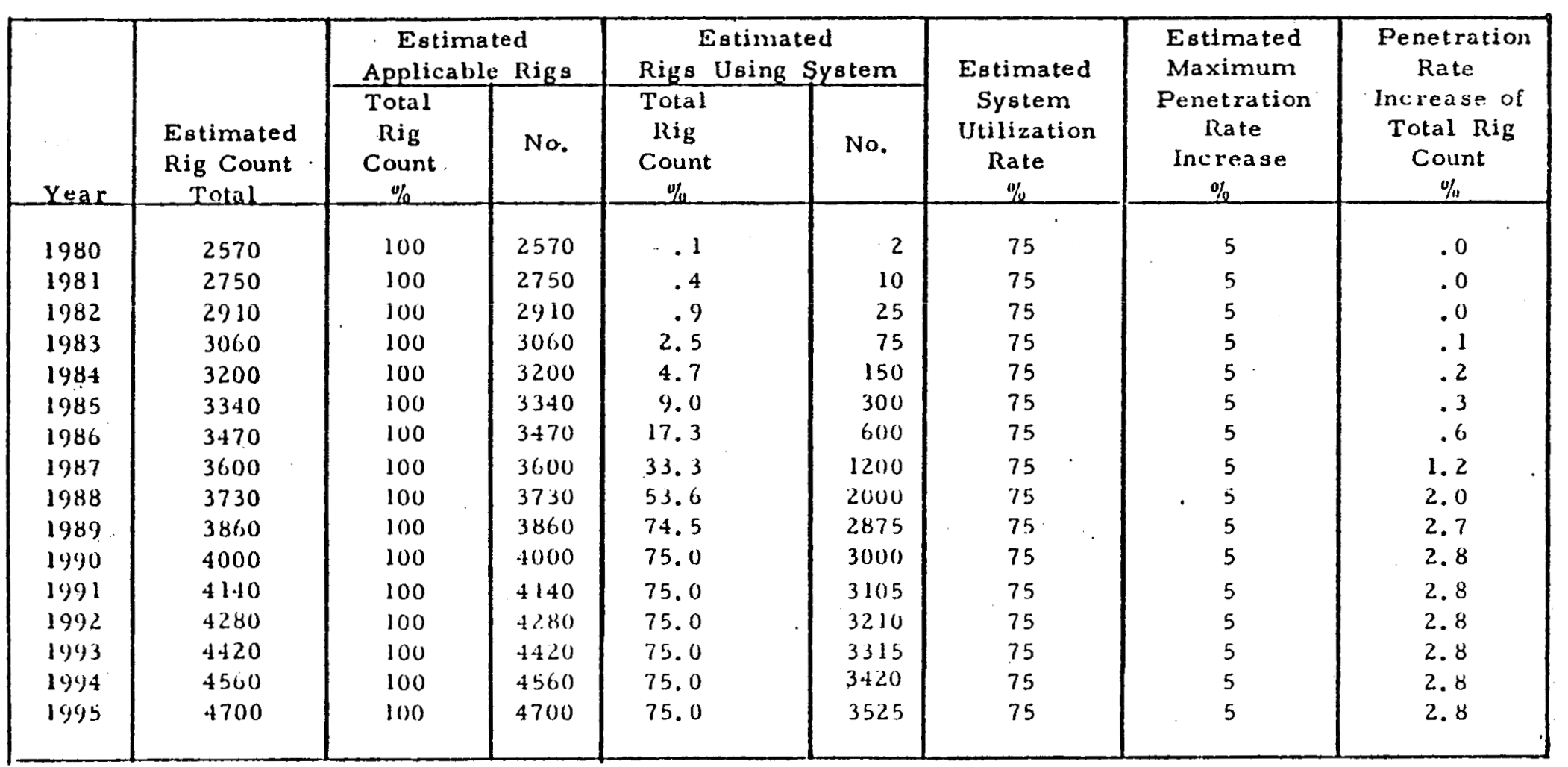

Estimated Rig Size Applicable: All Rigs

Estimated Maximum System Utilization Rate: $75 \%$

Estimated Maximum Penetration Rate Increase: $5 \%$ 
TABLE 5-13

PROJECT $3-3$, POTENTIAL

YEARLY PENETRATION RATE INCREASE (PERCENT)

\begin{tabular}{|c|c|c|c|c|c|c|c|c|}
\hline \multirow[b]{2}{*}{ Year } & \multirow[b]{2}{*}{$\begin{array}{c}\text { Estimated } \\
\text { Rig Count } \\
\text { Total }\end{array}$} & \multicolumn{2}{|c|}{$\begin{array}{l}\text { Estimated } \\
\text { Applicable Rigg }\end{array}$} & \multicolumn{2}{|c|}{$\begin{array}{c}\text { Estimated } \\
\text { Rigg Using Systern }\end{array}$} & \multirow{2}{*}{$\begin{array}{c}\text { Eatimated } \\
\text { System } \\
\text { Utilization } \\
\text { Rate } \\
\%\end{array}$} & \multirow{2}{*}{$\begin{array}{c}\text { Estimated } \\
\text { Maximum } \\
\text { Penetration } \\
\text { Rate } \\
\text { Increase } \\
\% \\
\end{array}$} & \multirow{2}{*}{$\begin{array}{l}\text { Penetration } \\
\text { Rate } \\
\text { Increase of } \\
\text { Total Rig } \\
\text { Count } \\
y_{11}\end{array}$} \\
\hline & & $\begin{array}{c}\text { Rig } \\
\text { Count } \\
\%\end{array}$ & No. & $\begin{array}{c}\text { Rig } \\
\text { Count } \\
\% \\
\end{array}$ & No. & & & \\
\hline 1980 & 2570 & 59 & 1516 &.$\quad .1$ & 2 & 50 & 15 & .0 \\
\hline 1981 & 2750 & 61 & 1678 & .2 & 5 & 50 & 15 & .0 \\
\hline 1982 & 2910 & 63 & 1833 & .3 & 10 & 50 & 15 & .0 \\
\hline 1983 & 3060 & 65 & .1989 & .5 & 15 & 50 & 15 & .0 \\
\hline 1984 & 3200 & 66 & 2112 & 0.9 & 30 & 50 & 15 & .1 \\
\hline 1985 & 3340 & 67 & 2238 & 1.8 & 60 & 50 & 15 & .1 \\
\hline 1986 & 3470 & 68 & 2360 & 3.5 & 120 & 50 & 15 & .3 \\
\hline 1987 & 3600 & 69 & 2484 & 6.9 & 250 & 50 & 15 & .5 \\
\hline 1988 & 3730 & 70 & 2611 & 10.7 & 400 & 50 & . 15 & .8 \\
\hline 1989 & 3860 & 71 & 2741 & 15.5 & 600 & 50 & 15 & 1.2 \\
\hline 1990 & 4000 & 72 & 2880 & 20.0 & 800 & 50 & 15 & 1.5 \\
\hline 1991 & 4140 & 73 & 3022 & 22.9 & 950 & 50 & 15 & 1.7 \\
\hline 1992 & 4280 & 74 & 3167 & 25.5 & 1090 & 50 & 15 & 1.9 \\
\hline 1993 & 4420 & 75 & 3315 & 26.2 & 1160 & 50 & 15 & 2.0 \\
\hline 1994 & 4560 & 76 & 3460 & 26.5 & 1210 & 50 & 15 & 2.0 \\
\hline 1945 & 4700 & 76 & 3572 & 26.6 & 1250 & 50 & 15 & 2.0 \\
\hline
\end{tabular}

Estimated Rig Size Applicable: $9-12^{\mathrm{K}}, 12-16^{\mathrm{K}}, 16^{\mathrm{K}}$-Up

Estimated Maximum System Utilization Rate: $50 \%$

Estimated Maximum Penetration Rate Increase: $15 \%$ 
PROJECT 3-4, POTENTIAL

YEARLY PENETRATION RATE INCREASE (PERCENT)

\begin{tabular}{|c|c|c|c|c|c|c|c|c|}
\hline \multirow[b]{2}{*}{ Year } & \multirow[b]{2}{*}{$\begin{array}{c}\text { Eatimated } \\
\text { Rig Count } \\
\text { Total }\end{array}$} & \multicolumn{2}{|c|}{$\begin{array}{c}\text { Estimated } \\
\text { Applicable Rigg }\end{array}$} & \multicolumn{2}{|c|}{$\begin{array}{c}\text { Eotimated } \\
\text { Rigs Using System }\end{array}$} & \multirow{2}{*}{$\begin{array}{c}\text { Estimated } \\
\text { System } \\
\text { Utilization } \\
\text { Rate } \\
\% \\
\end{array}$} & \multirow{2}{*}{$\begin{array}{c}\text { Estimated } \\
\text { Maximum } \\
\text { Penetration } \\
\text { Rate } \\
\text { Increase } \\
\text { or } \\
\end{array}$} & \multirow{2}{*}{$\begin{array}{c}\text { Penetration } \\
\text { Rate } \\
\text { Increase of } \\
\text { Total Rig } \\
\text { Count } \\
\% \\
\end{array}$} \\
\hline & & $\begin{array}{c}\text { Rig } \\
\text { Count } \\
\% \\
\end{array}$ & No. & $\begin{array}{c}\text { Rig } \\
\text { Count } \\
\% \\
\end{array}$ & No, & & & \\
\hline \\
\hline 1981 & & & & & & & & \\
\hline 1982 & & & & & & & & \\
\hline 1983 & & & & & & & & \\
\hline 1984 & 3200 & 82 & 2624 & .1 & 2 & 50 & 10 & .0 \\
\hline 1985 & 3340 & 83 & 2772 & .1 & 5 & 50 & 10 & .0 \\
\hline 1986 & 3470 & 83 & 2880 & .3 & 10 & 50 & 10 & .0 \\
\hline 1987 & 3600 & 84 & 3024 & .4 & 15 & 50 & 10 & .0 \\
\hline 1988 & 3730 & 84 & 3133 & .9 & 35 & 50 & 10 & .0 \\
\hline 1989 & 3860 & 85 & 3281 & 1.8 & 70 & 50 & 10 & .1 \\
\hline 1990 & 4000 & 85 & 3400 & 3.7 & 150 & 50) & 10 & .2 \\
\hline 1991 & 4140 & 86 & 3560 & 7.2 & 300 & 50 & 10 & .4 \\
\hline lys? & 4280 & 86 & 3687 & 12.9 & 550 & 50 & 10 & .6 \\
\hline 1993 & 4420 & 86 & 3801 & 18.1 & 800 & 50 & 10 & .9 \\
\hline 1994 & 4560 & 87 & 396,7 & 25.2 & 1150 & 50 & 10 & 1.3 \\
\hline 1995 & 4700 & 87 & 4089 & 30.4 & 1430 & 50 & 10 & 1.5 \\
\hline
\end{tabular}

Estimated Rig Size Applicable: $5-9^{K}, 9-12^{K}, 16^{K}-U p$ Estimated Maximum System Utilization Rate: $50 z$

Estimated Maximum Penetration Rate Increase: $10 \%$ 


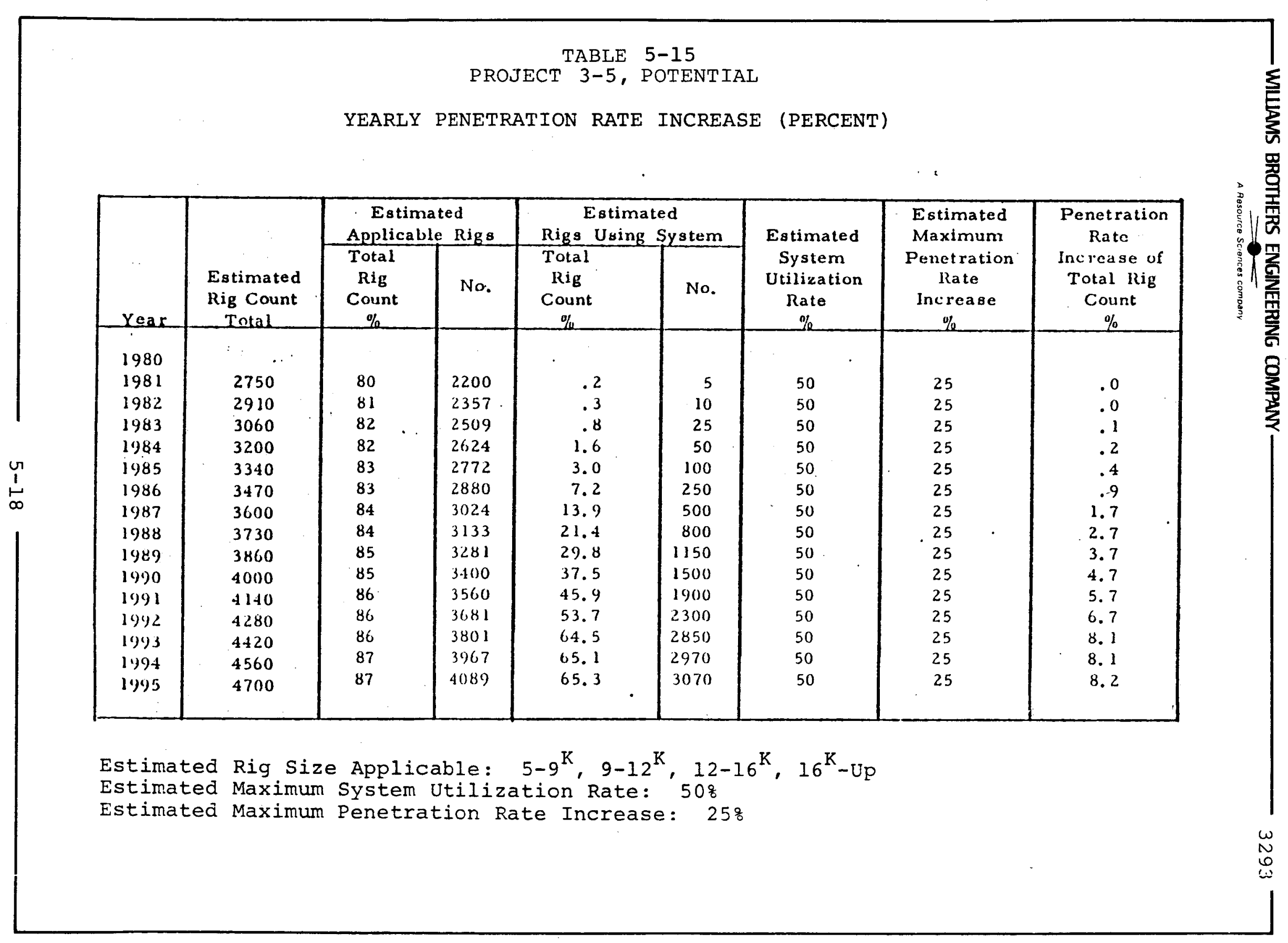




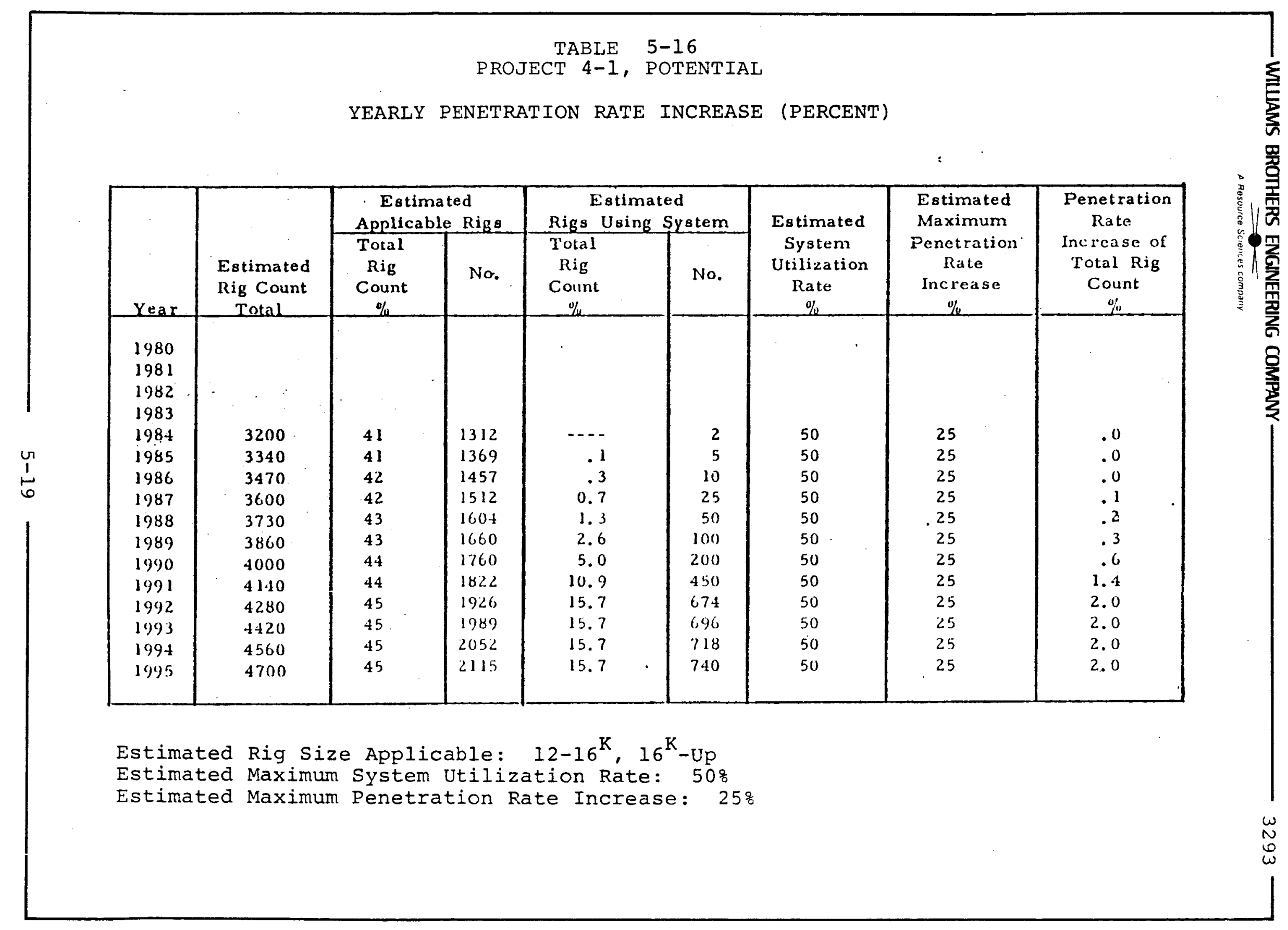


TABLE 5-17

PROJECT 5-1, POTENTIAL

YEARLY PENETRATION RATE INCREASE (PERCENT)

\begin{tabular}{|c|c|c|c|c|c|c|c|c|}
\hline \multirow[b]{2}{*}{ year } & \multirow[b]{2}{*}{$\begin{array}{c}\text { Estimated } \\
\text { Rig Count } \\
\text { Total }\end{array}$} & \multicolumn{2}{|c|}{$\begin{array}{c}\text { Estimated } \\
\text { Applicable Rigs }\end{array}$} & \multicolumn{2}{|c|}{$\begin{array}{c}\text { Estimated } \\
\text { Rigs Using System } \\
\end{array}$} & \multirow{2}{*}{$\begin{array}{c}\text { Estimated } \\
\text { System } \\
\text { Utilization } \\
\text { Rate } \\
\%\end{array}$} & \multirow{2}{*}{$\begin{array}{c}\text { Estimated } \\
\text { Maximum } \\
\text { Penetration } \\
\text { Rate } \\
\text { Increase } \\
\% \\
\end{array}$} & \multirow{2}{*}{$\begin{array}{c}\text { Penctration } \\
\text { Rate } \\
\text { Increase of } \\
\text { Total Rig } \\
\text { Count } \\
\% \\
\end{array}$} \\
\hline & & $\begin{array}{c}\text { Rig } \\
\text { Count } \\
\% \\
\end{array}$ & No. & $\begin{array}{c}\text { Rig } \\
\text { Count } \\
\%\end{array}$ & No. & & & \\
\hline \multirow{2}{*}{\multicolumn{9}{|c|}{$\begin{array}{l}1980 \\
1981\end{array}$}} \\
\hline & & & & & & & & \\
\hline 1982 & & & & & & & & \\
\hline 1983 & 3060 & 82 & 2509 & .1 & 2 & 50 & 25 & .0 \\
\hline 1984 & 3200 & 82 & 2624 & .2 & 5 & 50 & 25 & .0 \\
\hline 1985 & 3340 & 83 & 2772 & .3 & 10 & 50 & 25 & .0 \\
\hline 1986 & 3470 & 83 & 2880 & .7 & 25 & 50 & 25 & .1 \\
\hline 1987 & 3600 & 84 & 3024 & 1.4 & 50 & 50 & 25 & .2 \\
\hline 1988 & 3730 & 84 & 3133 & 2.7 & 100 & 50 & 25 & .3 \\
\hline 1989 & 3860 & 85 & 3281 & 5.2 & 200 & 50 & 25 & .6 \\
\hline 1990 & 4000 & 85 & 3400 & 11.2 & 450 & 50 & 25 & 1.4 \\
\hline 1991 & 4140 & 86 & 3560 & 16.9 & 700 & 50 & 2.5 & 2.1 \\
\hline 1992 & 4280 & 86 & 3081 & 21.0 & 900 & 50 & 25 & 2.6 \\
\hline 1ソ93 & 4420 & 86 & 3801 & 24.9 & 1100 & 50 & 25 & 3.1 \\
\hline 1994 & 4560 & 87 & 3967 & 30.4 & 1388 & 50 & 25 & 3.8 \\
\hline 1995 & 4700 & 87 & 4089 & 30.4 & 1431 & 50 & 25 & 3.8 \\
\hline
\end{tabular}

Estimated Rig Size Applicable: $9-12^{\mathrm{K}}, 12-16^{\mathrm{K}}, 16^{\mathrm{K}}-\mathrm{Up}$

Estimated Maximum System Utilization Rate: $50 \%$

Estimated Maximum Penetration Rate Increase: $25 \%$ 


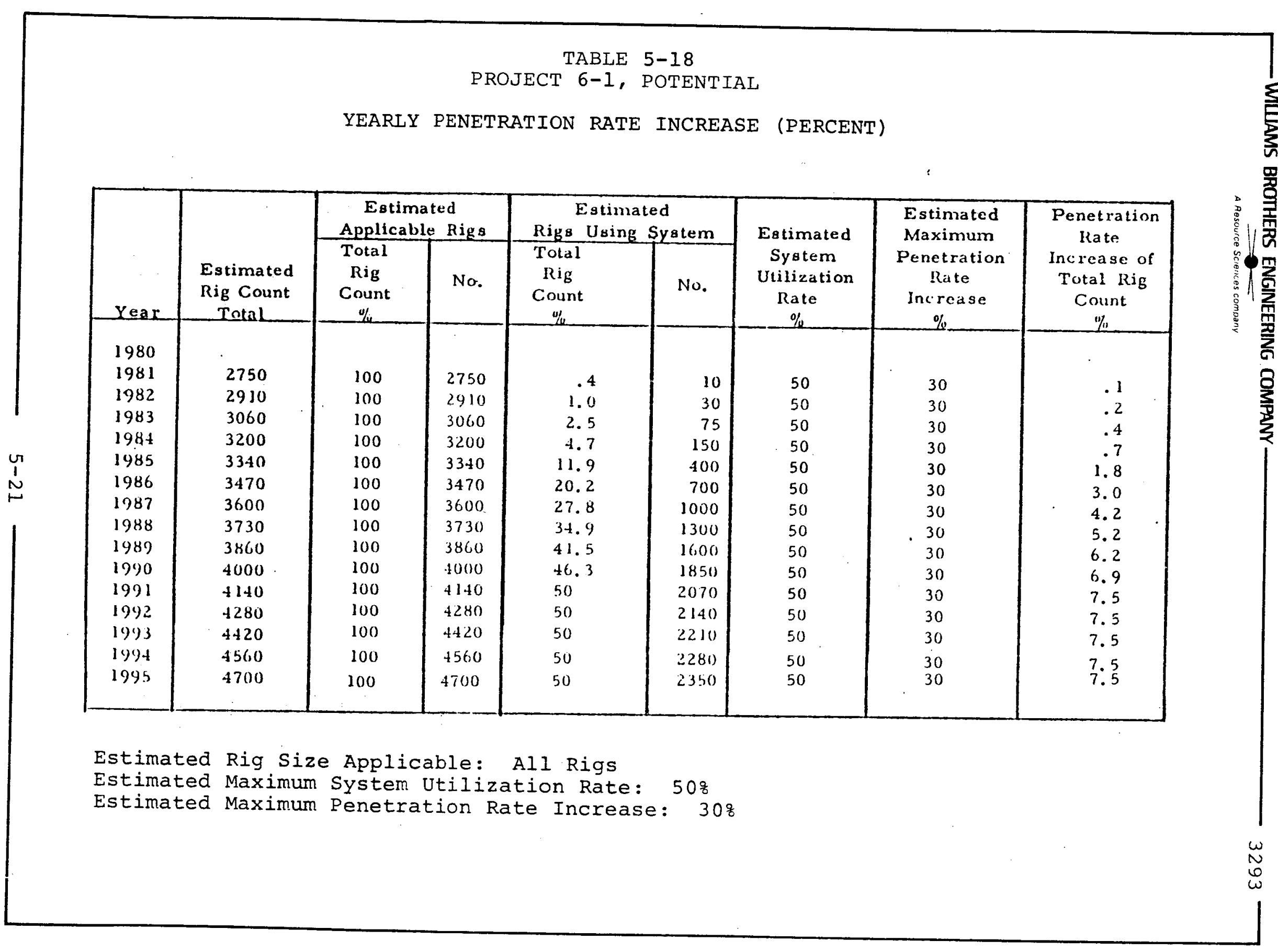


PROJECT 7-1, POTENTIAL

YEARLY PENETRATION RATE INCREASE (PERCENT)

\begin{tabular}{|c|c|c|c|c|c|c|c|c|}
\hline \multirow[b]{2}{*}{ Year } & \multirow[b]{2}{*}{$\begin{array}{c}\text { Estimated } \\
\text { Rig Count } \\
\text { Total }\end{array}$} & \multicolumn{2}{|c|}{$\begin{array}{c}\text { Estimated } \\
\text { Applicable Rigs }\end{array}$} & \multicolumn{2}{|c|}{$\begin{array}{c}\text { Estimated } \\
\text { Rigs Using System } \\
\end{array}$} & \multirow{2}{*}{$\begin{array}{c}\text { Estimated } \\
\text { System } \\
\text { Utilization } \\
\text { Rate } \\
\% \\
\end{array}$} & \multirow{2}{*}{$\begin{array}{c}\text { Estimated } \\
\text { Maximum } \\
\text { Penetration } \\
\text { Rate } \\
\text { Increase } \\
\% \\
\end{array}$} & \multirow{2}{*}{$\begin{array}{c}\text { Penetration } \\
\text { Rate } \\
\text { Increase of } \\
\text { Total Rig } \\
\text { Count } \\
\%\end{array}$} \\
\hline & & $\begin{array}{c}\text { Rig } \\
\text { Count } \\
\%\end{array}$ & $\mathrm{~N} r$. & $\begin{array}{c}\text { Rig } \\
\text { Count } \\
\%\end{array}$ & No. & & & \\
\hline 1980 & 2570 & & & & & 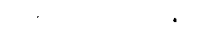 & & \\
\hline 1981 & 2750 & & & & & & & \\
\hline 1982 & 2910 & & & & & & & \\
\hline 1983 & 3060 & & & & & & & \\
\hline 1984 & 3200 & & & & & & & \\
\hline 1985 & 3340 & & & & & & & \\
\hline 1986 & 3470 & & & & & & & \\
\hline 1987 & 3600 & & & & & & & \\
\hline 1988 & 3730 & & & & & & & \\
\hline 1989 & 3860 & & & & & & & \\
\hline 1990 & 4000 & & & & & & & \\
\hline 1991 & $41+0$ & & & & & & & \\
\hline 1992 & 4280 & & & & & & & \\
\hline 1993 & 4420 & & & & & & & \\
\hline $\begin{array}{l}1994 \\
1995\end{array}$ & $\begin{array}{l}4560 \\
4700\end{array}$ & & & & & & & \\
\hline & & & & & & & & \\
\hline
\end{tabular}

Estimated Rig Size Applicable: $12-16^{\mathrm{K}}, 16^{\mathrm{K}}-\mathrm{Up}$

Estimated Maximum System Utilization Rate: Unknown

Estimated Maximum Penetration Rate Increase: -- 
PROJECT $8-1$, POTENTIAL

YEARLY PENETRATION RATE INCREASE (PERCENT)

\begin{tabular}{|c|c|c|c|c|c|c|c|c|}
\hline \multirow[b]{2}{*}{ Year } & \multirow[b]{2}{*}{$\begin{array}{c}\text { Eotimated } \\
\text { Rig Count } \\
\text { Total }\end{array}$} & \multicolumn{2}{|c|}{$\begin{array}{l}\text { Estimated } \\
\text { Applicable Rigg }\end{array}$} & \multicolumn{2}{|c|}{$\begin{array}{c}\text { Eatimated } \\
\text { Rigs Using System } \\
\end{array}$} & \multirow{2}{*}{$\begin{array}{c}\text { Estimated } \\
\text { System } \\
\text { Utilization } \\
\text { Rate } \\
\%\end{array}$} & \multirow{2}{*}{$\begin{array}{c}\text { Estimated } \\
\text { Maximum } \\
\text { Penetration } \\
\text { Rate } \\
\text { Increase } \\
\% \\
\end{array}$} & \multirow{2}{*}{$\begin{array}{c}\text { Penelration } \\
\text { Rate } \\
\text { Increase of } \\
\text { Total Rig } \\
\text { Count } \\
\end{array}$} \\
\hline & & $\begin{array}{c}\text { Rig } \\
\text { Count } \\
\text { uil: } \\
\end{array}$ & No. & $\begin{array}{c}\text { Rig } \\
\text { Count } \\
\% \\
\end{array}$ & No. & & & \\
\hline 1980 & 2570 & & & & & & & \\
\hline 1981 & 2750 & & & & & & & \\
\hline 1982 & 2910 & & & & & & & \\
\hline 1983 & 3060 & & & & & & & \\
\hline 1984 & 3200 & & & & & & & \\
\hline 1985 & 33.10 & & & & & & & \\
\hline 1986 & 3470 & & & & & & & \\
\hline 1987 & 3600 & & & & & & & \\
\hline 1988 & $\begin{array}{l}3730 \\
3460\end{array}$ & & & & & & & \\
\hline $\begin{array}{l}1989 \\
1090\end{array}$ & $\begin{array}{l}3860 \\
4000\end{array}$ & & & & & & & \\
\hline 1991 & 4140 & & & & & & & \\
\hline 1992 & 4280 & & & & & & & \\
\hline 1943 & 4420 & & & & & & & \\
\hline 1994 & 4560 & & & & & & & \\
\hline 1995 & 4700 & & & & & & & \\
\hline
\end{tabular}

Estimated Rig Size Applicable: $9-12^{\mathrm{K}}, 12-16^{\mathrm{K}}, 16^{\mathrm{K}}-\mathrm{Up}$

Estimated Maximum System Utilization Rate:

Estimated Maximum Penetration Rate Increase: -- 


\author{
APPENDIX A \\ DRILLING COMPANY ANALYSIS
}

The following tabulation of drilling companies shows the number of drilling rigs by type. Offshore rigs are shown as platform, mobile, or barge mounted.

The following abbreviations are included:

$$
\begin{aligned}
& \text { F - Foreign location of rig } \\
& \text { C - Cable tool rig } \\
& \text { W - Workover rig }
\end{aligned}
$$

Because rigs can be moved to and from foreign locations and workover rigs can be adapted for new hole production, this analysis should not be regarded as definitive. It does indicate make-up and size distribution of the drilling companies.

Sources: 1977 Oil \& Gas Journal "Land Drilling \& Oil Well servicing Contractors"

1977 Membership Directory of the International Association of Drilling contractors 


\begin{tabular}{|c|c|c|c|c|}
\hline \multirow{2}{*}{ COMPANY } & \multicolumn{4}{|c|}{ NUMBER OF RIGS } \\
\hline & 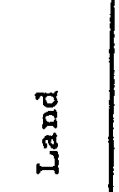 & $\underset{\substack{5 \\
\stackrel{5}{0}}}{\stackrel{0}{0}}$ & $\begin{array}{l}\stackrel{0}{\overrightarrow{0}} \\
\stackrel{0}{\circ}\end{array}$ & $\begin{array}{l}\text { 品 } \\
\text { 吕 } \\
\text { p. }\end{array}$ \\
\hline 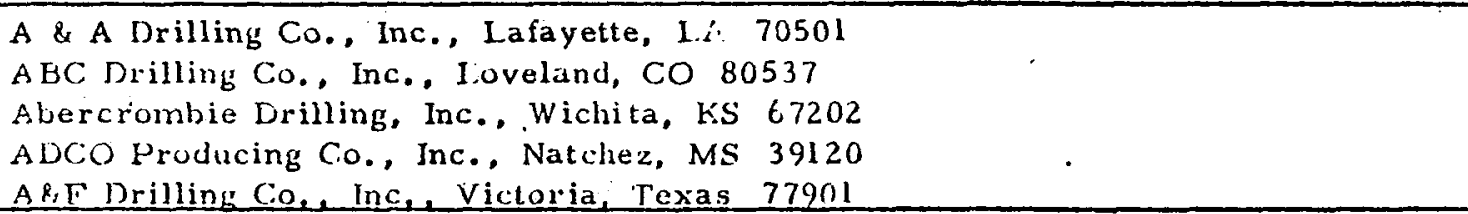 & $\begin{array}{l}3 \\
1 \\
5 \\
1\end{array}$ & & & \\
\hline $\begin{array}{l}\text { A \& P Drilling Company, Inc., Oklahoma City, OK } 73112 \\
\text { A B D Drilling Co., Wichita Falls, TX } 76301 \\
\text { AWI, Inc., Harvey, LA 70059 } \\
\text { ACCU Drill, Inc, , Lafayette, LA. } 70501 \\
\text { Aikins Drilling Co. Shelby, MT } 59474\end{array}$ & 2 & & & 3 \\
\hline $\begin{array}{l}\text { Alladin Drilling Activities A/S, Dreggen, Norway } \\
\text { Aladdin-Middle East, I.td., Wichita, KS } 67202 \\
\text { Alanco Drilling Co., Alice, TX } 78332 \\
\text { Allen, W. C., \& Sons, Shreveport, LA } \\
\text { Allison Drilling Co., Inc, Denver, CO } 80203 \\
\end{array}$ & $\begin{array}{l}1 \\
1\end{array}$ & & 2 & \\
\hline $\begin{array}{l}\text { Alpha Drilling, Inc. Edmond, OK } 73034 \\
\text { American Dilling, Inc., Lafayette, L.A. } 70505 \\
\text { American Natural Service Company, Detroit, MI } 48226 \\
\text { AMCQ Energy Corp., Dallas, TX } 75234 \\
\text { American Well Service \& Salvage. Inc., I afayette, I_A } 70505\end{array}$ & $\begin{array}{c}1 \\
2 \\
2+16 \\
2\end{array}$ & & & \\
\hline $\begin{array}{l}\text { American Well Services, Inc., Altus, OK } 75321 \\
\text { Amini Drilling Co. (See: KKA Drilling Co.) } \\
\text { Amoy Minerals Corp. San Antonio, TX } 78217 \\
\text { Amy Drilling Company. Opelousas, IA } 70570 \\
\text { Anders, Odell, Drilling Company, Midland, TX } 79701 \\
\end{array}$ & $3+3 W$ & & & \\
\hline 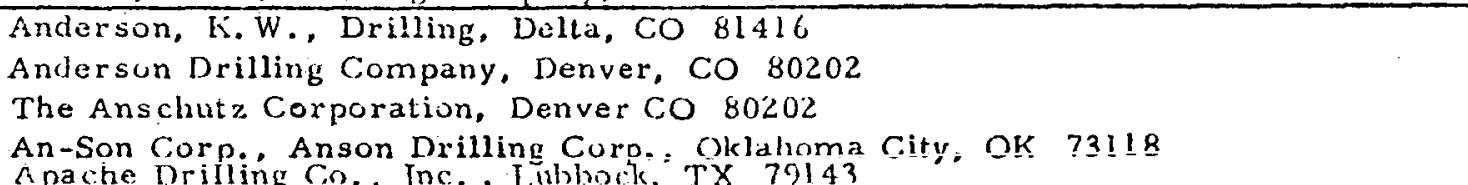 & $\begin{array}{r}2 \\
10\end{array}$ & $\underline{1}$ & & \\
\hline
\end{tabular}




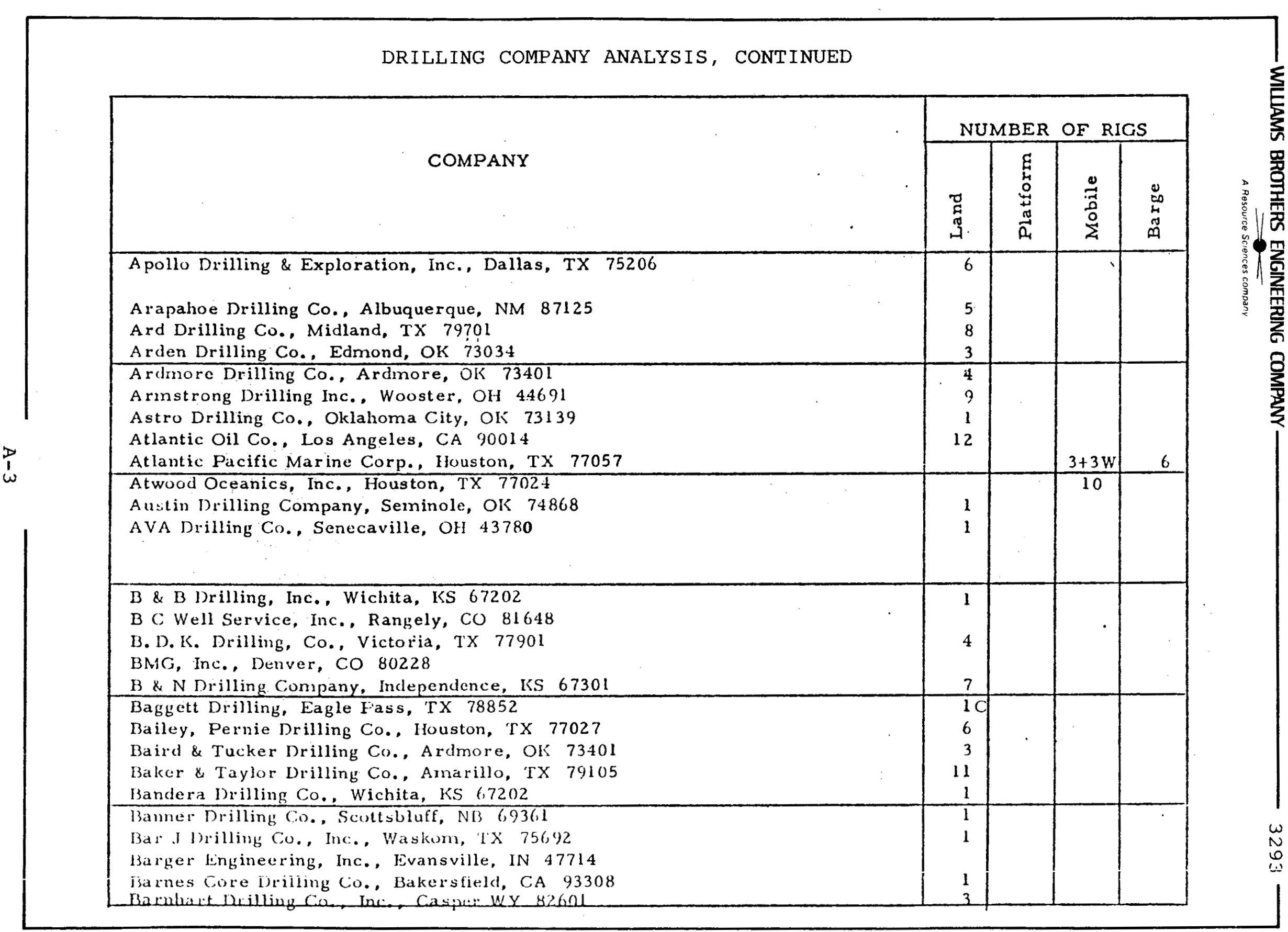


DRILLING COMPANY ANALYSIS, CONTINUED

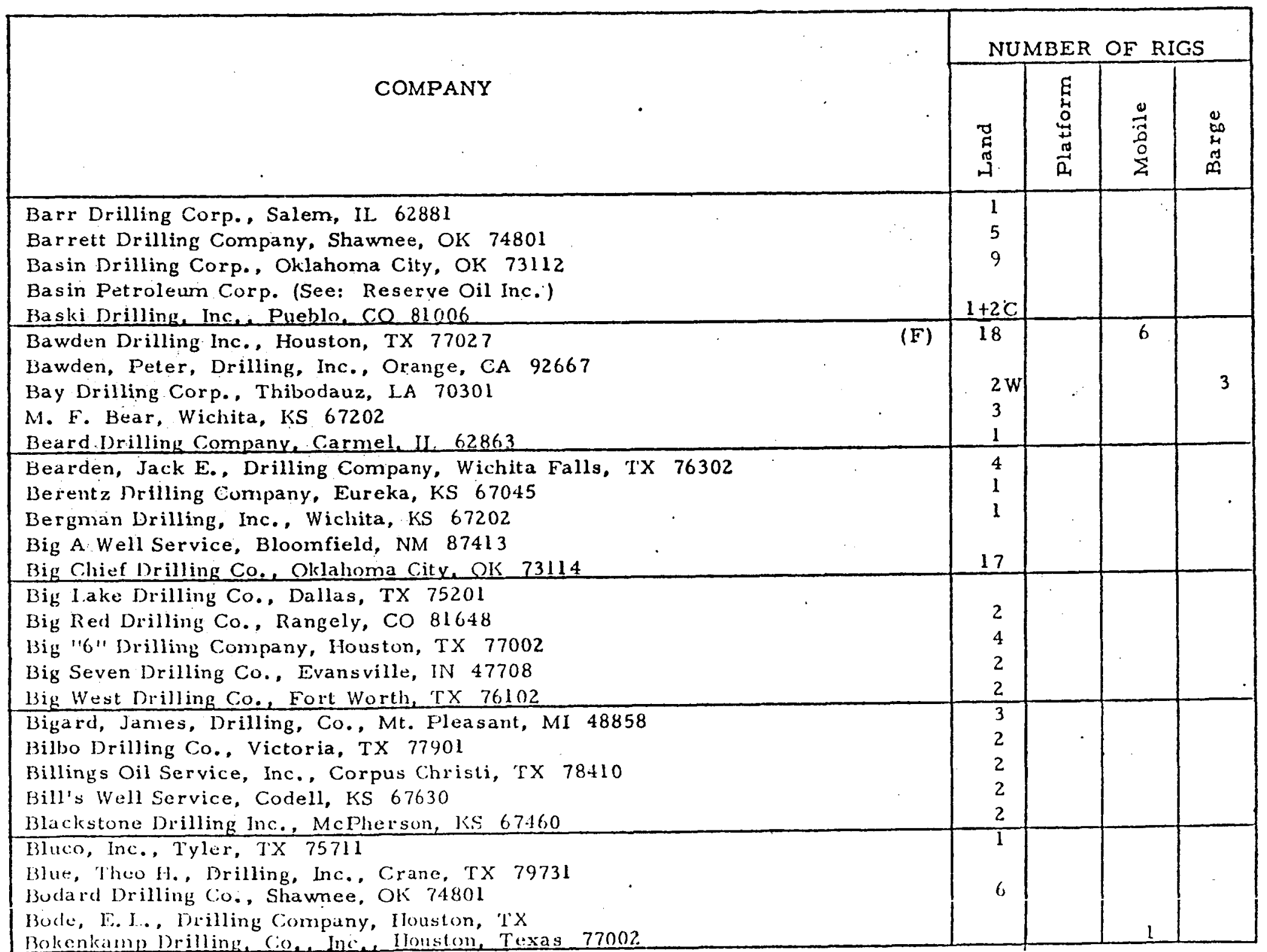


DRILLING COMPANY ANALYSIS, CONTINUED

\begin{tabular}{|c|c|c|c|c|}
\hline \multirow[b]{2}{*}{ COMPANY } & \multicolumn{2}{|c|}{ NUMBER } & \multicolumn{2}{|c|}{ OF RIGS } \\
\hline & 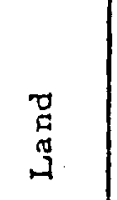 & 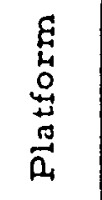 & 总 & $\begin{array}{l}0 \\
0 \\
\text { A } \\
0 \\
\text { m. }\end{array}$ \\
\hline $\begin{array}{l}\text { Bolin Oil Company, Wichita Falls, TX } 76301 \\
\text { Bomac Drilling, Lakewood, CO } 80215 \\
\text { Bonray Oil Company, Oklahoma City, OK } 73120 \\
\text { Booker Drilling Co., Inc., Metairie, LA } 70002 \\
\text { Boone, Wm. S., Drilling Contractor, Shreveport, LA } 71109\end{array}$ & $\begin{array}{r}3 \\
12 \\
7\end{array}$ & $16 \mathrm{~W}$ & 8 & \\
\hline $\begin{array}{l}\text { Boyd, Durst \& Kuenstler, Inc., Victoria, TX } 77901 \\
\text { Braden-Deem, Inc., Wichita, KS } 67202 \text {. } \\
\text { Brady Brothers Well Servicing Co., Cushing, OK } 74023 \\
\text { Raymond K. Brake Well Service, Fisher, II. } 61843 \\
\text { Brandt Drilling Co., Wichita, KS } 67202\end{array}$ & \begin{tabular}{l|}
4 \\
3 \\
$2 W$ \\
1 \\
1
\end{tabular} & & & \\
\hline $\begin{array}{l}\text { Brazzel Well Service, Inc., Lafayette, IA } 70501 \\
\text { C. E. Brehm Drilling \& Producing, Mt. Vernon, IL } 62864 \\
\text { "C" Brewer, Inc., Roundup, MT } 59072 \\
\text { Bridgcport Drilling Co., Inc., Olney, IL } 62450 \\
\text { Brinkerhoff Drilling Co., Inc., Denver CO } 80202\end{array}$ & $\ln$ & & & \\
\hline $\begin{array}{l}\text { Brothers Drilling Co.. Corpus Christi, TX } 78410 \\
\text { Brown \& Lawhorn Drilling Co. (See: Sundance Drilling Corp.) } \\
\text { Curt Brown Drilling Company, Tulsa, OK } 74103 \\
\text { Brown, Tom, Inc., Midland, TX } 79702 \\
\text { Brownie Drilling Co.. Lake Charles, LA } 70602\end{array}$ & $\begin{array}{c}3 \\
20 \\
1+4 \mathrm{~W} \\
\end{array}$ & & & \\
\hline $\begin{array}{l}\text { Bulold Drilling Company, Houston, TX } 77027 \\
\text { Bullock, A. O., Drilling Co., Casper, WY } 82601 \\
\text { Burger Drilling Co.; Lta., Abilene, TX } 79601 \\
\text { Burton Drilling Co., Houston, TX } 77002 \\
\text { Burton/Hawks Drlg. Co., Casper, WY } 82602\end{array}$ & $\begin{array}{l}1 \\
2 \\
1\end{array}$ & & & \\
\hline $\begin{array}{l}\text { Butler County Drilling Co.. Winfleld, ISS } 67156 \\
\text { Butler Drilling Co., Houston, TX 77021 } \\
\text { Butler-Johnson, Inc.. Bossier City, I.A } 71111 \\
\text { Buzzini Drilling Co., San Antonio, TX } 78209 \\
\text { Byed Drilling Co., Midland, TX 7970! } \\
\end{array}$ & $\begin{array}{r}2+10 \\
7 \\
8 \\
3 \\
7\end{array}$ & & & \\
\hline
\end{tabular}


DRILLING COMPANY ANALYSIS, CONTINUED

\begin{tabular}{|c|c|c|c|c|}
\hline \multirow{2}{*}{ COMPANY } & \multicolumn{2}{|c|}{ NUMBER } & \multicolumn{2}{|c|}{ OF RIGS } \\
\hline & $\begin{array}{l}\mathbf{T} \\
\mathbf{T} \\
\mathbf{H}\end{array}$ & 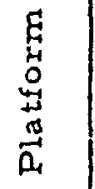 & $\begin{array}{l}\stackrel{0}{*} \\
\stackrel{0}{0} \\
\stackrel{0}{z}\end{array}$ & $\begin{array}{l}0 \\
\stackrel{\infty}{\infty} \\
\ddot{\omega} \\
\ddot{n}\end{array}$ \\
\hline $\begin{array}{l}\text { CBM Drilling Co., Houston, TX } 77006 \\
\text { C-G Drilling Co., Withita, KS } 67202 \\
\text { D \& L Drilling Company, Dallas, TX } 75202 \\
\text { C \& R Drilling Co., Perry, OK } 730,77 \\
\text { Cactus Drilling Co.e Richardson, TX } 75080 \\
\end{array}$ & $\begin{array}{c}2+1 W \\
1 \\
1 \\
2 \\
18 \\
\end{array}$ & & & \\
\hline $\begin{array}{l}\text { Caclus Drilling Corporation of Texas, Richardson, TX } 75080 \\
\text { California Prod. Service, Inc., Compton, Callfornia } 90224 \\
\text { Calvert Weslern Exploration Co., Fort Wortl, TX } 76102 \\
\text { Cam Drilling Co., Abilene, Tx } 79604 \\
\text { Camay Drilling Co. I.os Angeles, CA } 90010\end{array}$ & $\begin{array}{c}31 \\
2+10 w \\
6 \\
1 \\
10\end{array}$ & $1 \mathrm{~W}$ & & \\
\hline $\begin{array}{l}\text { Camden Drilling Company, Corpus Christi, } \Gamma \times 78401 \\
\text { Camrich Drilling Co., Tyler, TX } 75701 \\
\text { Cape Drilling Company, Tyler, TX } 75701 \\
\text { Caprock Drilling Service, Amarillo, TX } 79108 \\
\text { Captain Drilling Company, Inc., Odessa, TX } 79762 \\
\end{array}$ & $\begin{array}{l}5 \\
4\end{array}$ & & & $\overline{4}$ \\
\hline $\begin{array}{l}\text { Caraway, Frank, Drilling Co.. San Angelo, TX } 76901 \\
\text { Cardinal Drilling Company, Billings, MT } 59103 \\
\text { Carmack Drilling Co., Grand Junction, CO } 81501 \\
\text { Carmel, Inc.. Shreveport, I.A } 71165 \\
\text { Cedco Drilling Company, Olney, II. } 62450\end{array}$ & $\begin{array}{r}2 \\
11 \\
3 \\
7\end{array}$ & & . & \\
\hline $\begin{array}{l}\text { Centreville Fetroleum, Ine., Ilarvey, I.A } 70058 \\
\text { Century Drilling Company, Shelby, MT } 59474 \\
\text { Challenger Drilling Inc., Calgary, Alta, Canada } \\
\text { Chandler \& Associates, Inc. Denver, CO. } 80202\end{array}$ & $\begin{array}{l}3 \\
2 \\
2\end{array}$ & & & 2 \\
\hline 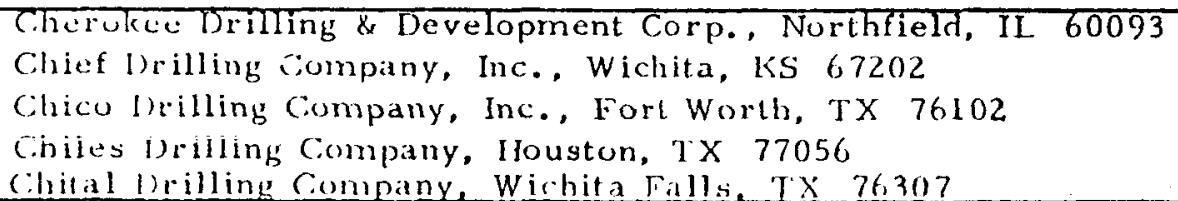 & $\begin{array}{l}2 \\
1 \\
4\end{array}$ & & 1 & \\
\hline
\end{tabular}


DRILLING COMPANY ANALYSIS, CONTINUED

\begin{tabular}{|c|c|c|c|c|}
\hline \multirow[b]{2}{*}{ COMPANY } & \multicolumn{4}{|c|}{ NUMBER OF RIGS } \\
\hline & 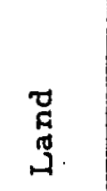 & 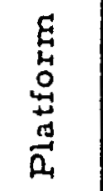 & 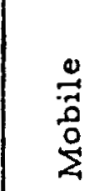 & 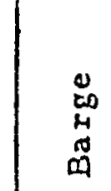 \\
\hline $\begin{array}{l}\text { Choya Drilling Company, Alice, TX } 78332 \\
\text { Cinco Drilling Company, Corpus Christi, TX } 78401 \\
\text { Circle "A" Drilling Co., Denver, GO } 80202 \\
\text { Circle Bar Drilling Company. New Orleans. I.A } 70112\end{array}$ & $\begin{array}{r}10 \\
4 \\
7\end{array}$ & & & 1 \\
\hline $\begin{array}{l}\text { Circle G Drilling Company, Bridgeport, TX } 76026 \\
\text { E. B. Clark, Sr., Wichita Falls, TX } 76301 \\
\text { Clawsun, Don, Drilling Contractor, Ada, OK } 74820 \\
\text { Claybrook Drilling Co., Inc., Wewoka, OK } 74884 \\
\text { Clem Corporation, The, Houston, TX } 77002\end{array}$ & $\begin{array}{l}1 \\
1 \\
1 \\
3 \\
1\end{array}$ & $\cdot$ & & \\
\hline $\begin{array}{l}\text { George B. Cole \& Sons, Benton Harbor, MI } 49022 \\
\text { Coleman Drilling Co., Farmington, NM } 87401 \\
\text { Columbia Drilling Co., Houston, IX } 77098 \\
\text { Comet Drilling Co., Lafayette, LA } 70505 \\
\text { Commanche Drilling Co., Cut Bank. MT } 59427\end{array}$ & $\begin{array}{l}4 \\
6 \\
2 \\
3 \\
4\end{array}$ & & & $1+1 W$ \\
\hline $\begin{array}{l}\text { Copenhaver Drilling Co., Victoria, TX } 77901 \text { Craneco, Farmington, NM } 87401 \\
\text { Crescent Drilling Company. Owensbory. KY } 42301 \\
\text { I) rescent Well Service, Inc., Harvey, IA } 70058 \\
\text { Croatian Drilling Co.. Inc., Harvey, I.A } 70058 \\
\text { Crom, IBob, Inc., }\end{array}$ & $\begin{array}{l}2 \\
4 \\
2 W\end{array}$ & & & \\
\hline $\begin{array}{l}\text { Crow-Quaid Enterprises, Seal Beach, CA } 90740 \\
\text { Cyclone Drilling Inc.. Gillette, WY } 82716 \\
\text { Cyclops Drilling Co., New Orleans, IA } 70112\end{array}$ & $\begin{array}{l}1 \\
2\end{array}$ & 2 & & 1 \\
\hline 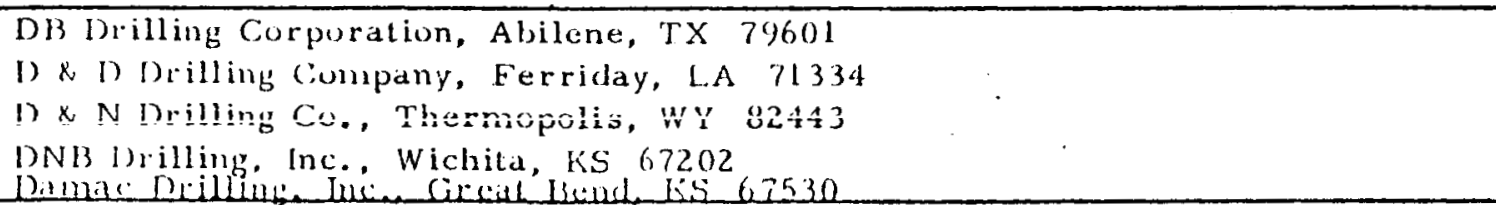 & $\begin{array}{r}11 \\
3\end{array}$ & & & \\
\hline
\end{tabular}

DNB l) rilling. Inc. Wichila KS 67202

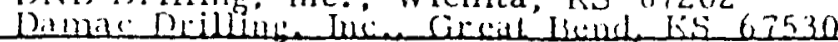


DRILLING COMPANY ANALYSIS, CONTINUED

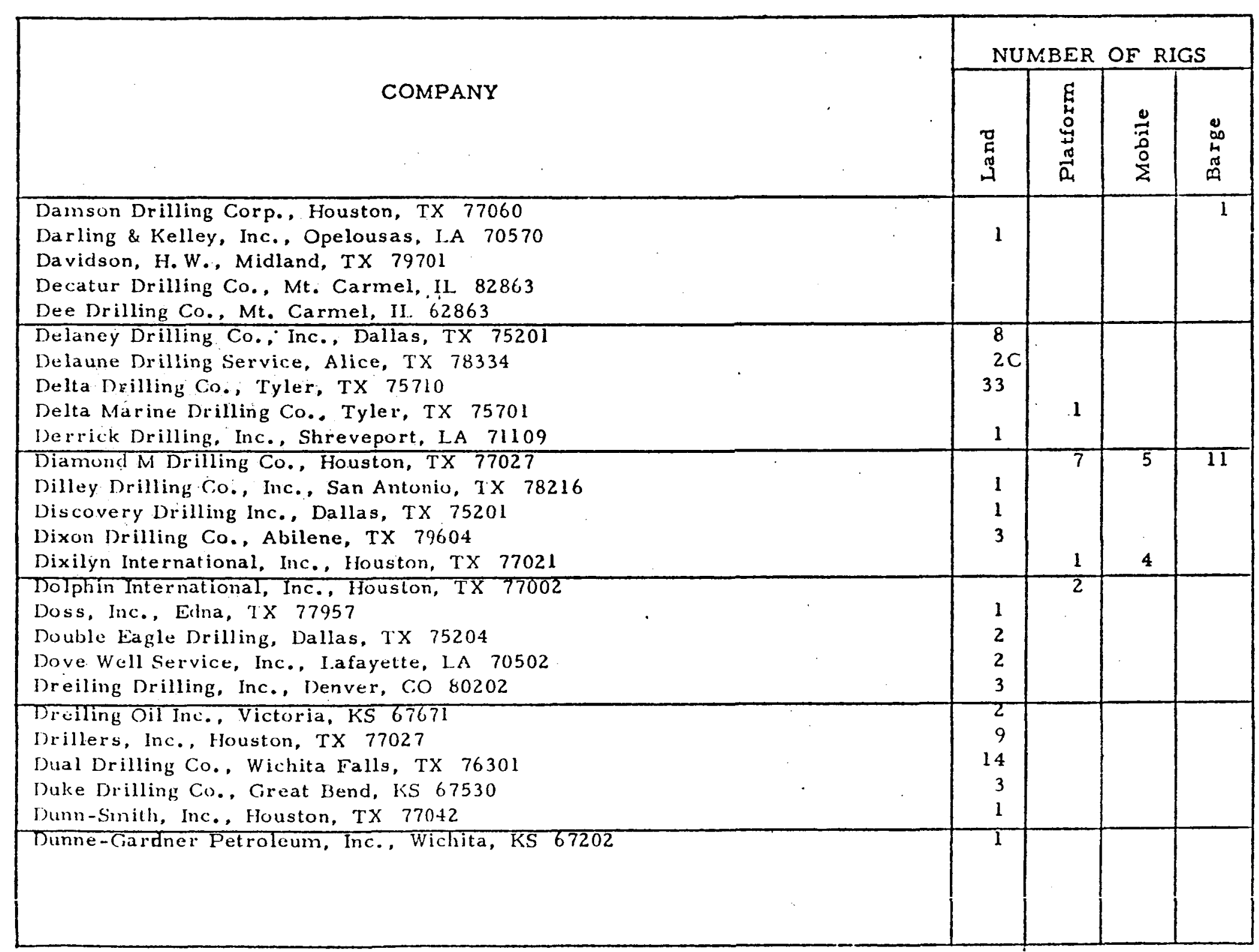


DRILLING COMPANY ANALYSIS, CONTINUED

\begin{tabular}{|c|c|c|c|c|}
\hline \multirow[b]{2}{*}{ COMPANY } & \multicolumn{4}{|c|}{ NUMBER OF RIGS } \\
\hline & 总 & 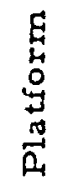 & $\begin{array}{l}\stackrel{0}{\stackrel{1}{*}} \\
\stackrel{0}{0} \\
\stackrel{\Delta}{\Sigma}\end{array}$ & 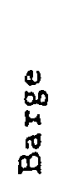 \\
\hline $\begin{array}{l}\text { E \& H Drilling Co., Graham, TX } 76046 \\
\text { ESI Drilling, Inc., Moab, UT } 84532 . \\
\text { Eagle Drilling Co., Natchez, MS } 39120 \\
\text { Eason Oil Company, Oklahoma Cily, OK } 73118 \\
\text { Edico Drillers, Inc., Liberal, KS } 67901 \\
\end{array}$ & $\begin{array}{l}2 \\
1 \\
\end{array}$ & & & \\
\hline $\begin{array}{l}\text { Eggleston, A.W., Inc, . Crowley, LA } 70526 \\
\text { Eklund Drilling Co., Carlin, NV } 89822 . \\
\text { El Dorado Drilling, Inc. O Oklahoma City, OK } 73112 \\
\text { Elenburg Exploration, Inc., Havre, MT } 59501 \\
\text { El Gato Drilling Company, Dallas, TX } 75201 \\
\end{array}$ & $\begin{array}{l}1 \\
4 \\
2 \\
\end{array}$ & & & \\
\hline $\begin{array}{l}\text { Empire Drilling Co., Denver, CO } 80207 \\
\text { Energy Drillers, Inc., Ft. Worth, TX } 76102 \\
\text { Roy Ensminger, Chanute, KS } 88720 \\
\text { Euphoria Oil Corporation, Alexandria, VA } 22302 \\
\text { Evertson Well Service, Inc., Kimball, NB } 69145\end{array}$ & \begin{tabular}{l|}
$2 W$ \\
2 \\
1 \\
7
\end{tabular} & r & & \\
\hline $\begin{array}{l}\text { Evitt Drilling \& Service Co., Ft. Lupton, CO } 80621 \\
\text { Exeter Drilling Northern, Inc., Denver, CO } 80264^{\circ} \\
\text { Exeter Drilling Southern, Inc., New Orleans, LA } 70112 \\
.\end{array}$ & \begin{tabular}{r|}
2 \\
23 \\
4
\end{tabular} & & & \\
\hline $\begin{array}{l}\text { F-W Drilling Co., Abilene, TX } 79605 \\
\text { FWA Drilling Company, Inc. Wichita Falls, Texas } 76301 \\
\text { Failman Drilling Co., Du Bois, PA } 15801 \\
\text { Faith Drilling Co., Houston, TX 77001 } \\
\text { Farmer, John O., Inc., Russell, KS } 67665 \\
\end{array}$ & $\begin{array}{r}3 \\
25 \\
7 \\
2 \\
1\end{array}$ & & & \\
\hline 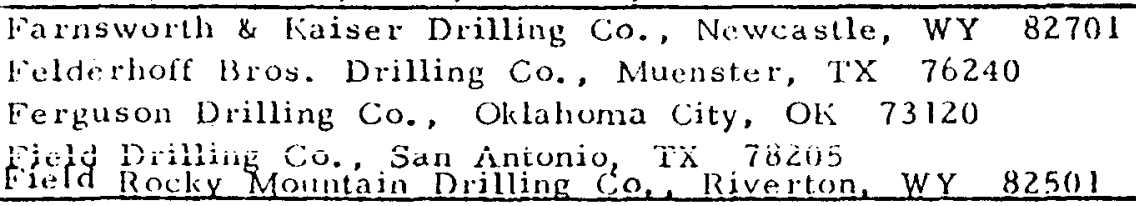 & $\begin{array}{r}10 \\
15 \\
2 \\
\end{array}$ & & 5 & i \\
\hline
\end{tabular}




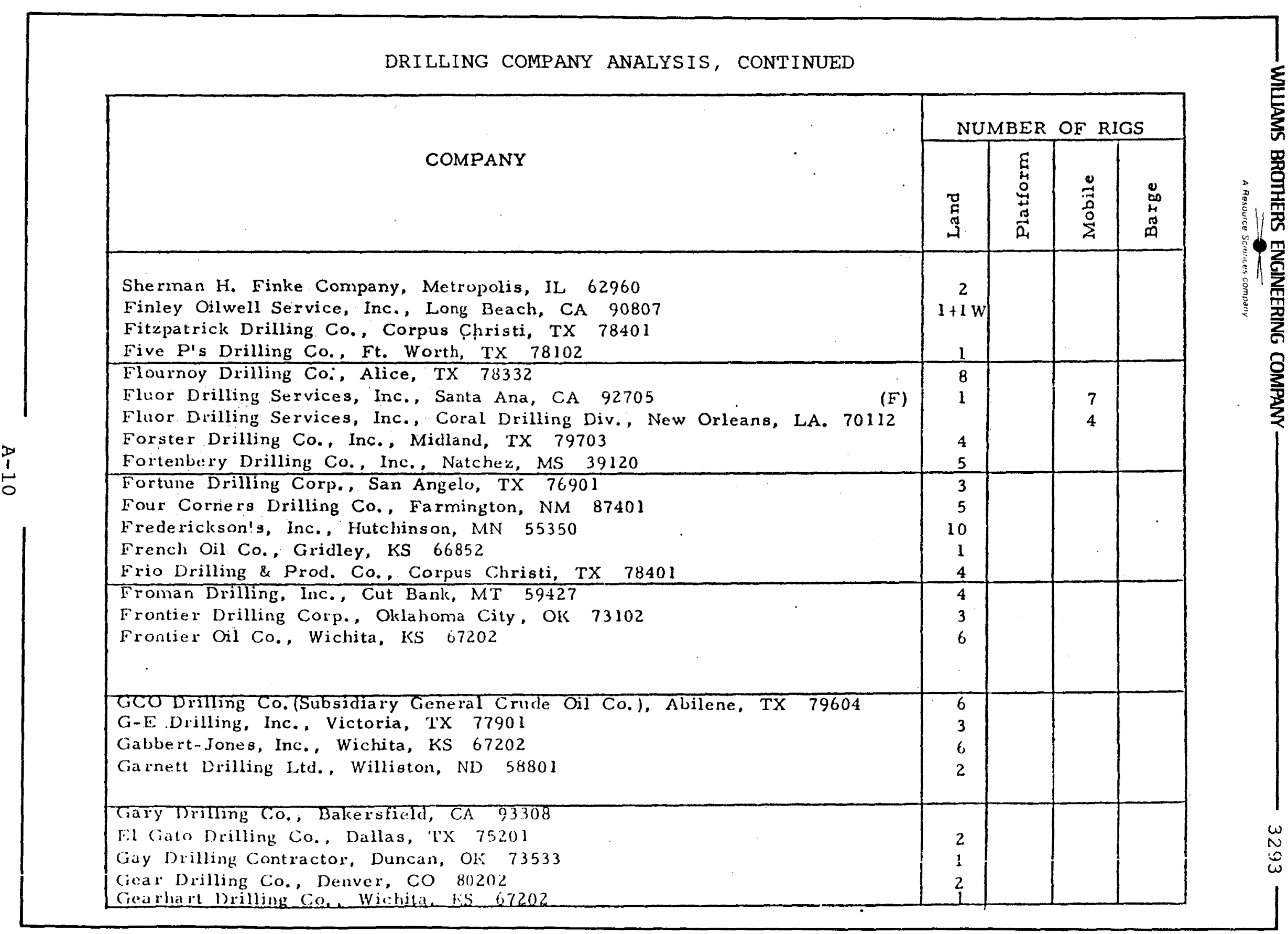




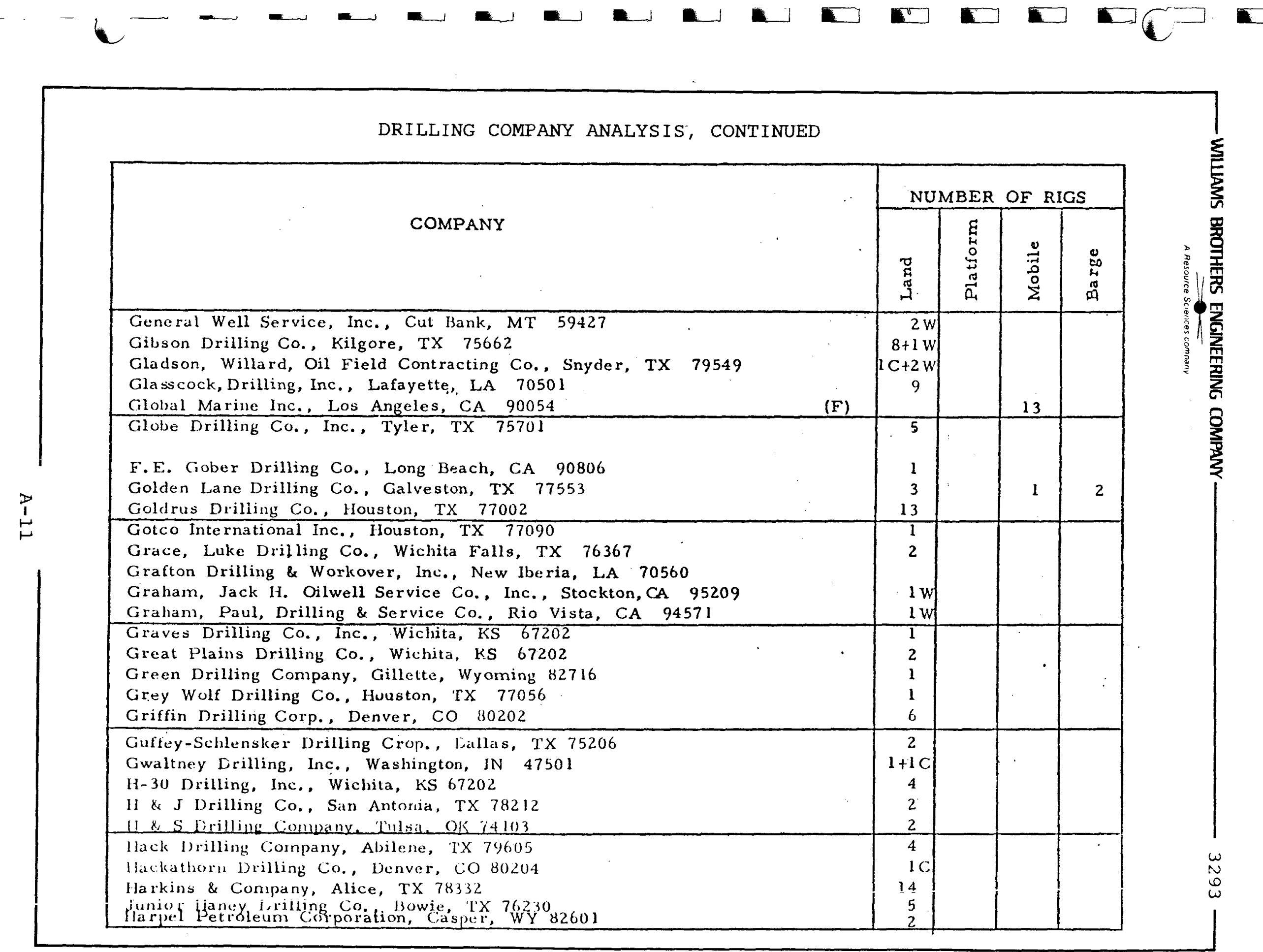


DRILLING COMPANY ANALYSIS, CONTINUED

\begin{tabular}{|c|c|c|c|c|}
\hline \multirow{2}{*}{ COMPANY } & \multicolumn{2}{|c|}{ NUMBER } & \multicolumn{2}{|c|}{ OF RIGS } \\
\hline & 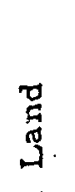 & 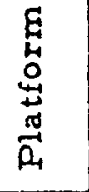 & 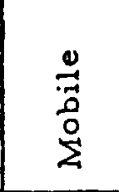 & 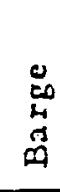 \\
\hline $\begin{array}{l}\text { Harper-Turner Drilling, Div., Harper Oil Company, Okla. City, OK } 73102 \\
\text { Harris Drilling Company, Grayville, IL } 62844 \\
\text { llatcher, H. F. \& Son, Smackover, AR } \\
\text { Hauge Brothers, Conrad, MT 59425 } \\
\text { Hleadwaters Oil Company, Dallas, TX } 75235 \\
\end{array}$ & $\begin{array}{l}4 \\
1 \\
2\end{array}$ & & $\checkmark$ & \\
\hline $\begin{array}{l}\text { lelmerich \& Payne, Inc., Tulsa, OK } 74114 \\
\text { llerb Exploration Company, Abilene, TX } 79604 \\
\text { lerring, Maxwell Drilling Corporation, Tyler, TX } 75710 \\
\text { Hickman Drilling Co., Woodward, OK } 73801 \\
\text { llill, Jim B. Dililing Co., Ventura, CA } 92003\end{array}$ & $\begin{array}{r}22 \\
1 \\
6 \\
2 \\
1\end{array}$ & & & \\
\hline $\begin{array}{l}\text { Hlillin Drilling Co., Odessa, TX } 79760 \\
\text { Hinton, W. B., Drilling Co., Inc., Mt. Pleasant, TX } 75455 \\
\text { Hogan Drilling Co., Baton Rouge, LA } 70806 \\
\text { Holliday Drilling Co., Midland, TX } 79702 \\
\text { Holmes Drilling Co., Houston, TX } 77002 \\
\end{array}$ & $\begin{array}{l}7 \\
6 \\
1 \\
2\end{array}$ & & & \\
\hline $\begin{array}{l}\text { Holston Well Services, Inc., Jennings, LA } 70546 \\
\text { Hondo Drilling Co., Midland, TX 79702 } \\
\text { - Honeymon Drilling Co. Ltd., Oklahoma City, OK } 73107 \\
\text { Hoover Drilling Co., Bakersfield, CA } 93308 \\
\text { Houston, Jack Exploration Co., Dallas, TX } 75230 \\
\end{array}$ & $\begin{array}{l}4 \\
7 \\
3 \\
1 \\
1\end{array}$ & & & \\
\hline $\begin{array}{l}\text { lloustom Offshore International, Inc., Houston, TX } 77027 \\
\text { Ilowell Drilling, Inc. San Antonio, TX } 78205 \\
\text { Iludson, Leonard Drilling Company, Inc. Pampa, TX } 79065 \\
\text { llughes Drilling Company, Fnid, OK } 73701 \\
\text { Ilunnicutt \& Camp Drilling Company, Rio Vista, CA 9457) }\end{array}$ & $\begin{array}{l}2 \\
6 \\
4 \\
5\end{array}$ & & 1 & \\
\hline $\begin{array}{l}\text { Ifuthance Drilling Company, Houston, TX } 77002 \\
\text { llylion Drilling Company, Bakerstield, CA } 93304\end{array}$ & 5 & 1 & & \\
\hline
\end{tabular}

Harper-Turner Drilling, Div, Harper Oil Company, Okla. City, OK 73102

Headwaters Oil Company, Dallas, TX 75235

Hickman Drilling Co, Woodward, OK 73801

Hillin Drining Co. Odessa, TX 79760

Hint on, W. B., Drilling Co., Inc., Mt. Pleasant, TX 75455

llolmes Drilling Co. Houston, TX 77002

Ilolston Well Services, Inc., Jennings, LA 70546

Hover Drilling Co., Bakersfield, CA 93308

Hotlston, Jack Exploration Co., Dallas, TX 75230

Ilowell Drilling, Inc. San Antonio, TX 78205

llughes Drilling Company, Fnicl, OK 73701

Iluthance Drilling Company, Houston, TX 77002 
DRILLING COMPANY ANALYSIS, CONTINUED

\begin{tabular}{|c|c|c|c|c|}
\hline \multirow[b]{2}{*}{ COMPANY } & \multicolumn{4}{|c|}{ NUMBER OF RIGS } \\
\hline & 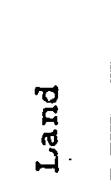 & 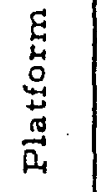 & $\begin{array}{l}\stackrel{0}{ت} \\
\stackrel{ت}{0} \\
\stackrel{2}{\Sigma}\end{array}$ & $\begin{array}{l}\stackrel{0}{c} \\
\text { 峁 } \\
\tilde{m}\end{array}$ \\
\hline $\begin{array}{l}\text { Inland Well Service, Abbeville, LA } 70510 \\
\text { Intairdril Ltd., Houston, TX } 77063\end{array}$ & $\begin{array}{r}4 \\
25\end{array}$ & & 3 & $5 W$ \\
\hline $\begin{array}{l}\text { J-V Drilling Company, Inc., Evansville, IN } 47710 \\
\text { J \& C Drilling Company, Inc., Refugio, TX } 78377 \\
\text { Jackson, L. B., Co., Tulsa, OK } 74135 \\
\text { Jernigan Drilling Company, Oklahoma City, OK } 73112 \\
\text { Jet Drilling Co., Inc., Shreveport, LA } 71101\end{array}$ & $\begin{array}{c}9 \\
1 \\
1+1 C \\
1 \\
7 \\
\end{array}$ & & & \\
\hline $\begin{array}{l}\text { John Drilling Company, Odessa, TX } 79762 \\
\text { Johns on-Bates Drilling Company, Konawa, OK } 74849 \\
\text { Johnston Drilling Company, Houston, TX } 77043 \\
\text { Jones Bros. Drilling Co, Inc., Shreveport, LA } 71101 \\
\text { Jones Drilling \& Producing Co., Fairfield, Il, } 62837 \\
\end{array}$ & \begin{tabular}{c|}
4 \\
6 \\
$9 \mathrm{C}$ \\
$2+1 \mathrm{C}$ \\
\end{tabular} & & & \\
\hline $\begin{array}{l}\text { Jones Drilling Corporation, Duncan, OK } 73533 \\
\text { Justiss-Mears Oil Company, Inc., Jena, LA } 71342 \\
\text { KKA Drilling Company, Midland, TX 79701 } \\
\text { Kay Drilling Co., Ponca City, OK } 74601 \\
\text { Kellogg, K.L. \& Sons, Long Beach, CA } 90807\end{array}$ & \begin{tabular}{r|}
9 \\
21 \\
5 \\
1 \\
1
\end{tabular} & & & \\
\hline $\begin{array}{l}\text { Kenai Drilling Limited, New York, NY } 10022 \\
\text { Kendall Drilling Company, Inc. } \\
\text { Kern Drilling Co. Inc. Magnolia, AR } 71753 \\
\text { Key International Drilling Company Limited, Houston, TX } 77002 \\
\text { Keydril Company, Houston, TX } 77002 \\
\end{array}$ & $\begin{array}{r}18 \\
3 \\
5\end{array}$ & & $\begin{array}{l}3 \\
3\end{array}$ & \\
\hline 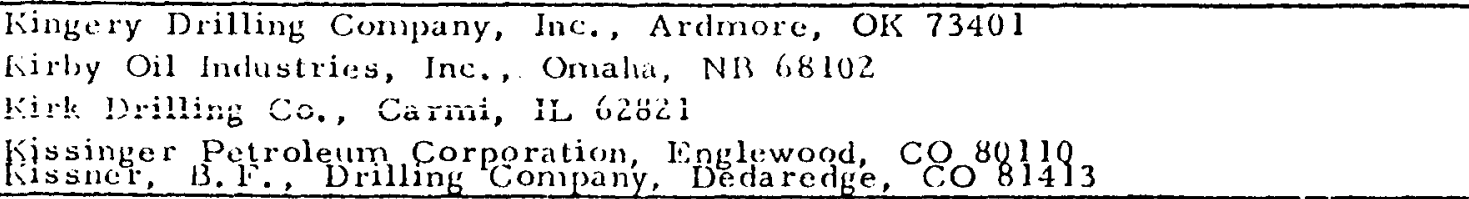 & $\begin{array}{ll}2 \\
1 C\end{array}$ & & & \\
\hline
\end{tabular}




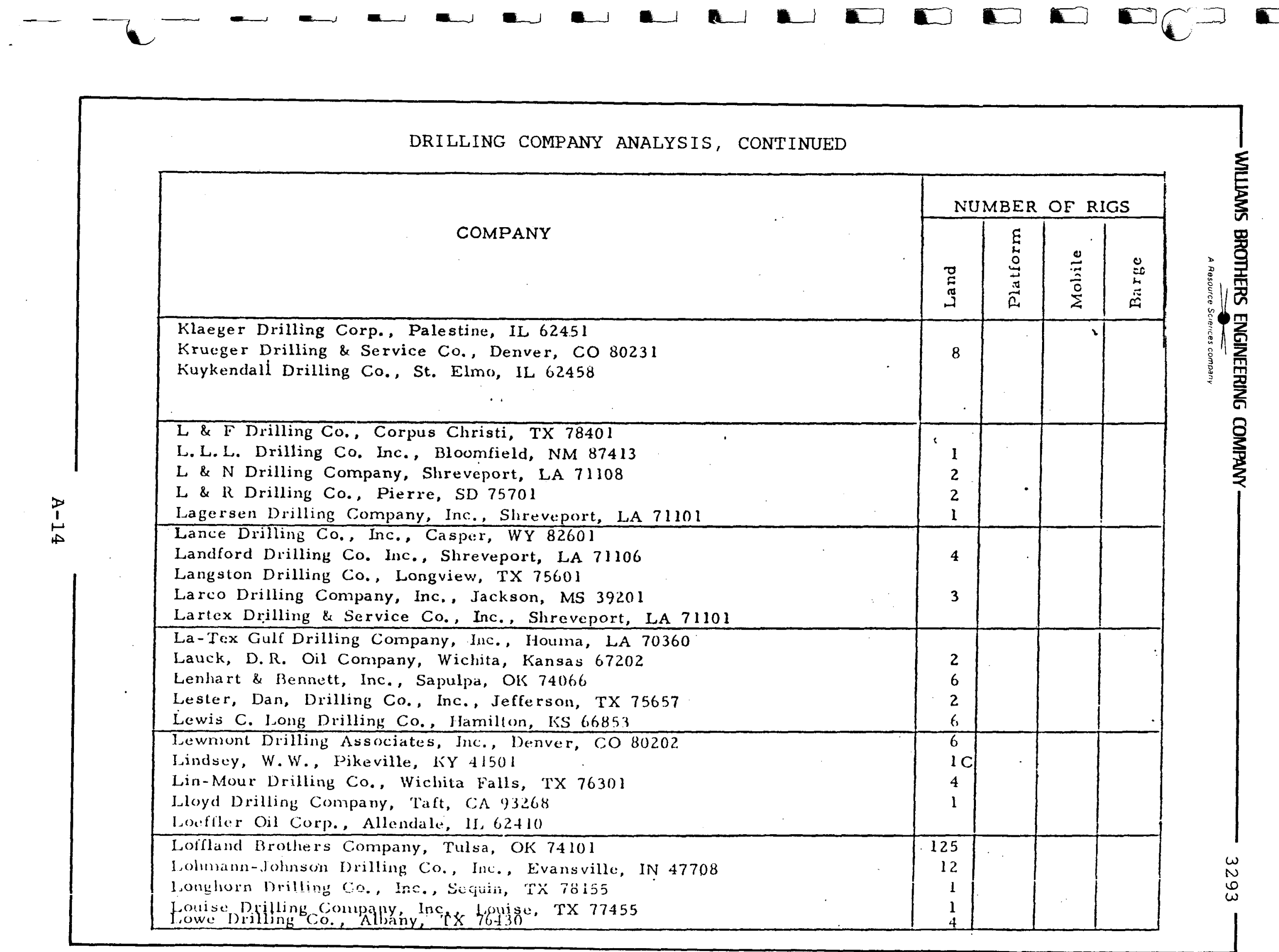


DRILLING COMPANY ANALYSIS, CONTINUED

\begin{tabular}{|c|c|c|c|c|}
\hline \multirow[b]{2}{*}{ COMPANY } & \multicolumn{4}{|c|}{ NUMBER OF RIGS } \\
\hline & 胥 & 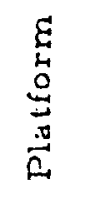 & 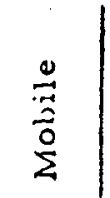 & $\underset{ت}{\stackrel{0}{*}}$ \\
\hline $\begin{array}{l}\text { McLeman Drilling Co., Nowata, OK } 74048 \\
\text { McVay Drilling Company, Tulsa, OK } 74103 \\
\text { Megargel Drilling Co., Inc., Megargel, TX } 76370 \\
\text { Melland Drilling Co., Inc. McPherson, KS } 67460 \\
\text { Mclton Drilling Company, Bakersfield, CA } 93308 \\
\end{array}$ & $\begin{array}{l}2 \\
5 \\
6 \\
1 \\
2\end{array}$ & & 2 & \\
\hline $\begin{array}{l}\text { Melton, Joe, Driliing Co., Inc., Midland, TX 79701 } \\
\text { Mercury Drilling Co., Tulsa, OK } 74114 \\
\text { Mesa Drillers, Dolores, CO } 81323 \\
\text { Mid-Continent Oil \& Gas Corporation, Downey, CA 90241 } \\
\text { Midstales Drilling Company, Rising Star, TX 76471 }\end{array}$ & $\begin{array}{r}1 \\
1 \\
3 \\
2\end{array}$ & & & \\
\hline $\begin{array}{l}\text { Milam Drilling Co., San Antonio, TX } 78205 \\
\text { Miller Brothers Drilling Corp., Allegan, MI } 49010 \\
\text { Miller Drilling Company, Ft. Smith, AR } 72906 \\
\text { Marjon F. Miller Drilling Co., St. Peter, IL } 62880 \\
\text { Minor, F. M. Drilling Company, Olney, IL } 62450 \\
\end{array}$ & $\begin{array}{l}2 \\
9 \\
2 \\
\end{array}$ & & & \\
\hline $\begin{array}{l}\text { Mission Drilling \& Exploration Corporation, New Orleans, LA } 70139 \\
\text { Mitchell, George N. Drlg. Co., Carmi, IL } 62821 \\
\text { Molen Drilling Co., Inc., Billings, MT 59103 } \\
\text { Montgomery, R. B., Drilling, Inc, Bakersfield, CA } 93303 \\
\text { Moran Bros., Inc., Wichita Falls, TX } 76301 \\
\end{array}$ & $\begin{array}{r}4 \\
3 \\
8 \\
16 \\
\end{array}$ & & 1 & \\
\hline $\begin{array}{l}\text { Moran, E. W., Drilling Company, Inc, Wichita Falls, TX } 76301 \\
\text { Moran, F. E. Oil Co., Owensboro, KY } 42301 \\
\text { Moranco, llobbs, N. M. B2240 } \\
\text { Morco Drilling, Inc, Corpus Cluristi, TX } 78408 \\
\text { Morgan Drilling Co., Shreveport, LA } 71106\end{array}$ & $\begin{array}{l}7 \\
1\end{array}$ & & & \\
\hline 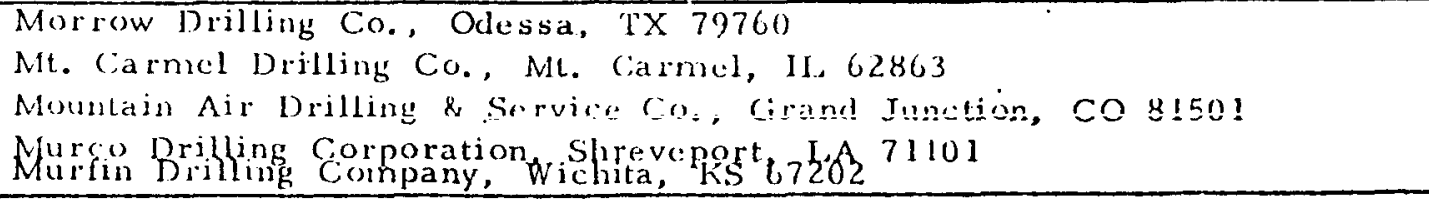 & $\begin{array}{r}1 \\
9 \\
6+16 \\
\end{array}$ & & & \\
\hline
\end{tabular}


DRILLING COMPANY ANALYSIS, CONTINUED

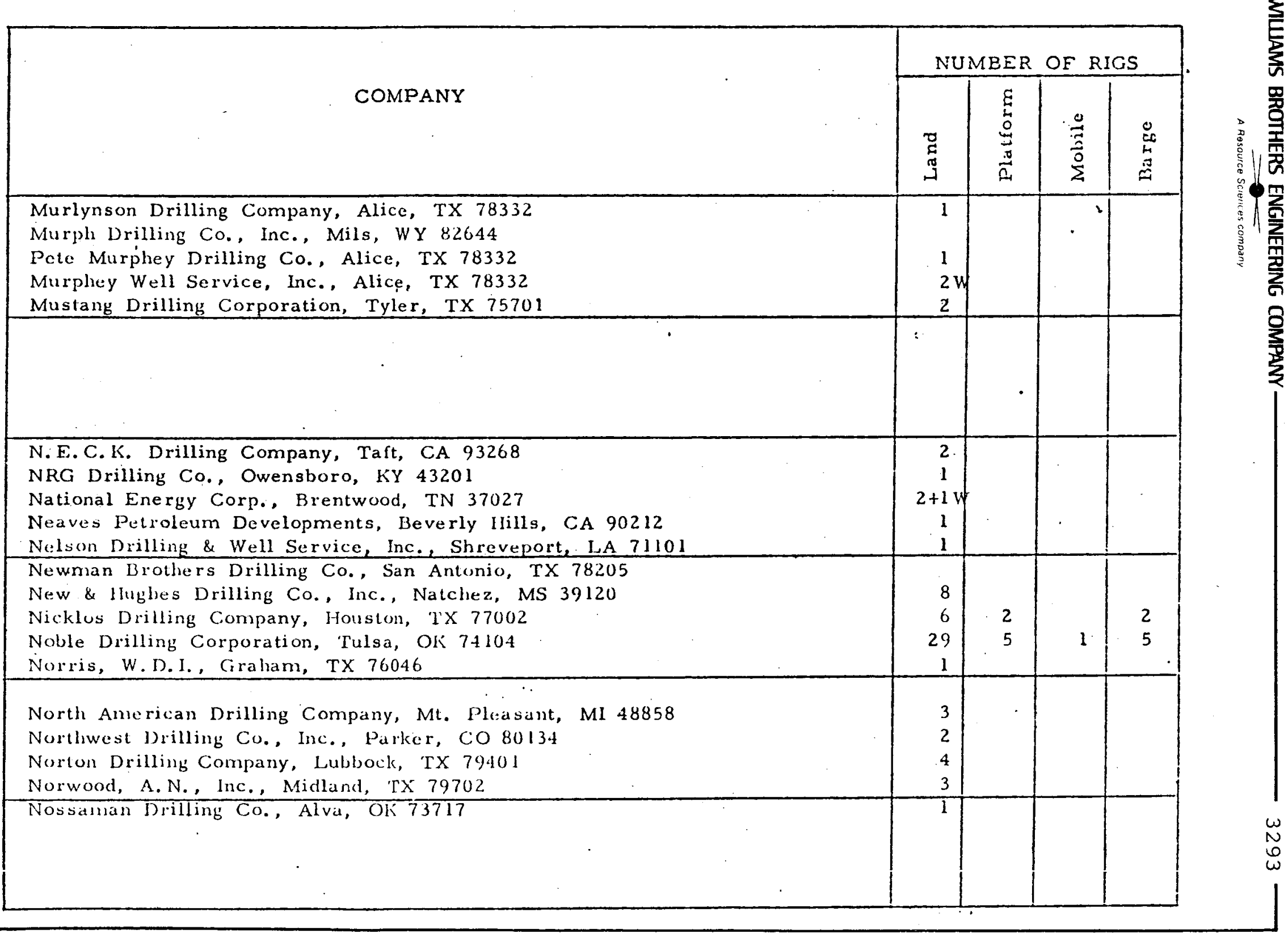


DRILLING COMPANY ANALYSIS, CONTINUED

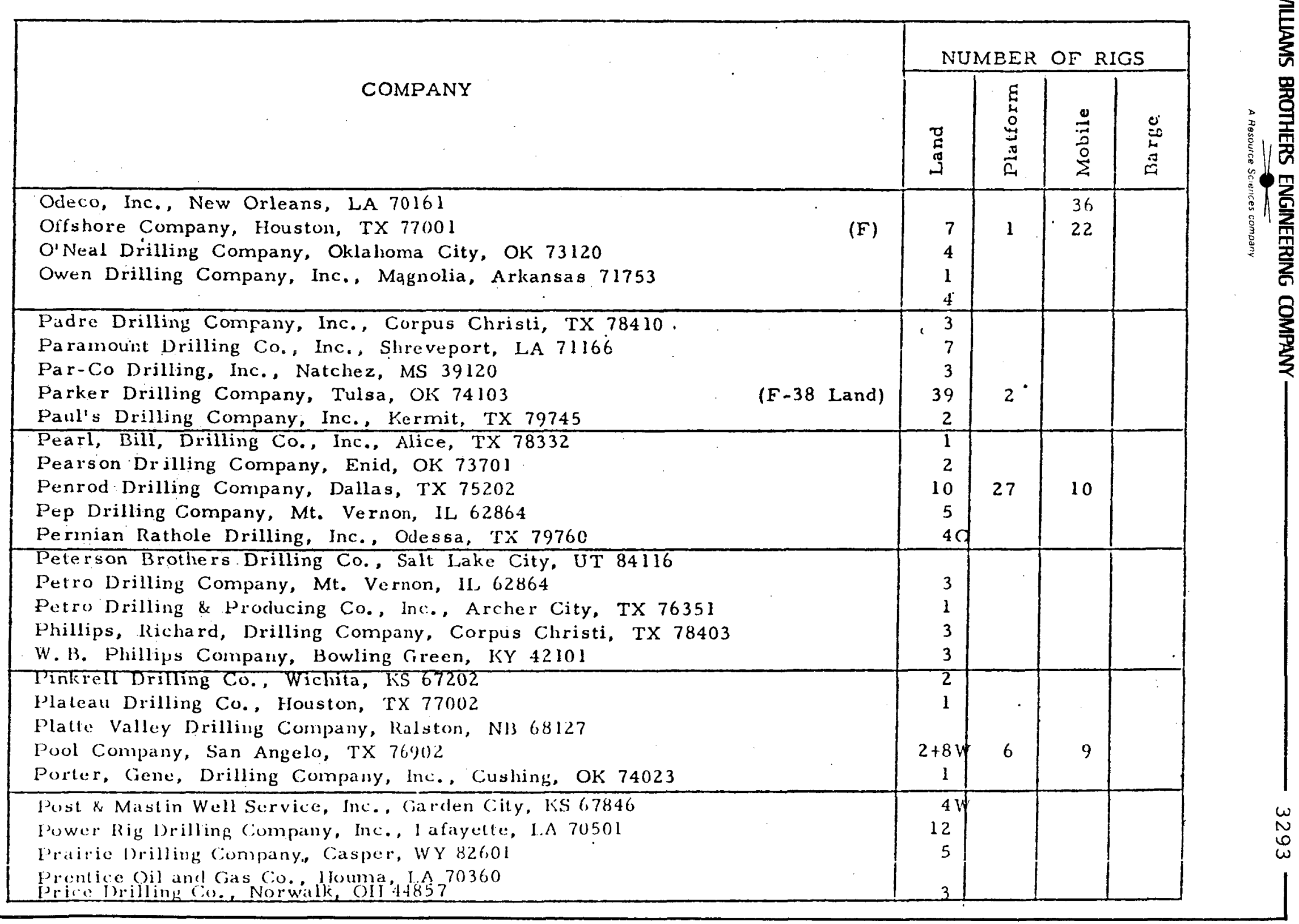


DRILLING COMPANY ANALYSIS, CONTINUED

\begin{tabular}{|c|c|c|c|c|}
\hline \multirow[b]{2}{*}{ COMPANY } & \multicolumn{4}{|c|}{ NUMBER OF RIGS } \\
\hline & 苐 & 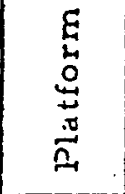 & 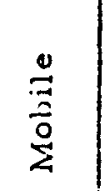 & 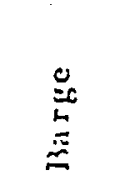 \\
\hline $\begin{array}{l}\text { Prince Bruthers Drilling Company, Electra, TX } 76360 \\
\text { Progress Prilling \& Marine, Inc., Houston, TX } 77027 \\
\text { Pronghorn Drilling, Glenrock, WY } 82637 \\
\text { Prulential Drilling Co., Houston, TX } 77027 \\
\text { Pruet, Chesley. Drilling Co., El Dorado, Arkansas } 71730 \\
\end{array}$ & $\begin{array}{r}2 \\
28 \\
4 \\
\end{array}$ & $6+8 w$ & 13 & 3 \\
\hline $\begin{array}{l}\text { Pyburn Drilling Co., Shreveport, LA } 71104 \\
\text { PliD Drilling Co., Houston, } T X \text {. } \\
\text { Pease Drilling, Grand Junction, Co } \\
\text { Pennsylvania Drilling Co., Pittsburgh, PA }\end{array}$ & $\begin{array}{r}2 \\
2 \\
5 \\
10\end{array}$ & & & \\
\hline $\begin{array}{l}\text { Quad Drilling Corporation, Bossier City, LA } 71010 \\
\text { Quasar, Incorporated, Evansville, IN } 47715 \\
\text { Ray Resources, Charleston, W. VA. }\end{array}$ & 2 & & & \\
\hline $\begin{array}{l}\text { R K Petroleum Corp., Mt. Carmel, IL } 62863 \\
\text { Rains \& Williamson Oil Co., Inc., Wichita, KS } 67202 \\
\text { Ratliff Drilling Company, Duncan, OK } 73533 \\
\text { Reading \& Bates Drilling Co., Houston, TX } 77002 \\
\text { Belistock Drilling Company. Kennel. IA } 70062\end{array}$ & $\begin{array}{r}1+1 d \\
7 \\
10\end{array}$ & 2 & 2 & $1+2 W$ \\
\hline $\begin{array}{l}\text { Red Tiger Drilling Company, Wi chita, KS } 67202 \\
\text { Reid Drilling Company. Casper, WY } 82601 \\
\text { Remco Drilling Corp. Haughton, LA } 71037 \\
\text { Republic: Cieothermal, Inc., Santa le Springs, CA } 90670 \\
\text { Reserve Oil, Inc., OkJahoma Cily, OK } 73112\end{array}$ & \begin{tabular}{c|}
6 \\
1 \\
$1+3$ \\
9
\end{tabular} & & & \\
\hline 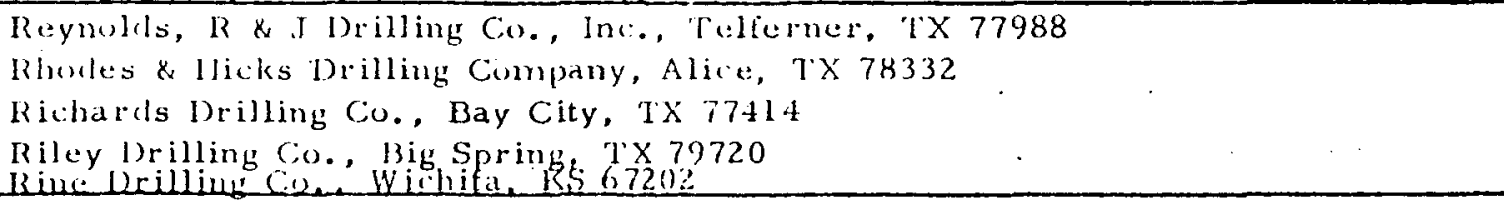 & $\begin{array}{l}1 \\
2 \\
5 \\
3 \\
7\end{array}$ & & & \\
\hline
\end{tabular}


DRILLING COMPANY ANALYSIS, CONTINUED

\begin{tabular}{|c|c|c|c|c|}
\hline \multirow{2}{*}{ COMPANY } & \multicolumn{4}{|c|}{ NUMBER OF RIGS } \\
\hline & 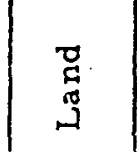 & 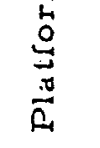 & $\stackrel{0}{\stackrel{\bar{\Xi}}{\Sigma}}$ & 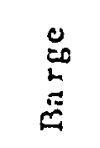 \\
\hline $\begin{array}{l}\text { Roberts Drilling, Inc., Casper, WY } 82601 \\
\text { Robinson Brothers Drilling Co., Midland, TX } 79701 \\
\text { Robinson Drilling of 'Texas, Inc., Big Spring, TX } 79720 \\
\text { Rockhold Engineering, Inc., Great Bend, KS } 67530 \\
\text { Rod Ric Corporation, Midland, TX } 79701\end{array}$ & $\begin{array}{l}5 \\
4 \\
2 \mathrm{C} \\
5 \\
\end{array}$ & & 4 & \\
\hline $\begin{array}{l}\text { Roden Drilling Co., Casper, WY } 82601 \\
\text { Rosamund, Phillip Drilling Co., Inc. West Monroe, LA } 71291 \\
\text { Rutary Drilling, Inc., Tyler, TX } 75701 \\
\text { Rowan Companies, Inc., Houston, TX } 77056 \\
\text { Rwit Drilling, Holdenville, OK }\end{array}$ & $\begin{array}{r}4 \\
2 \\
12 \\
3 \\
\end{array}$ & $4^{\circ}$ & 1 & \\
\hline $\begin{array}{l}\text { R.O. Roy, Shreveport, I.A } \\
\text { Sardex Drilling, Kaysville, UT }\end{array}$ & 1 & & & \\
\hline $\begin{array}{l}\text { S \& H Drilling Company, Inc., Victoria, TX } 77901 \\
\text { S H \& J Drilling Corporation, Magnolia, AR } 71753 \\
\text { S \& W Drilling' Company, Inc. , Lawrenceville, IL. } 62439 \\
\text { Subre Drilling, Cushing, OK } 74023 \\
\text { Sabre Drilling Co., Ince, Billings, Montana } 52102 \\
\end{array}$ & $\begin{array}{l}2 \\
3 \\
5 \\
3 \\
1\end{array}$ & & & \\
\hline 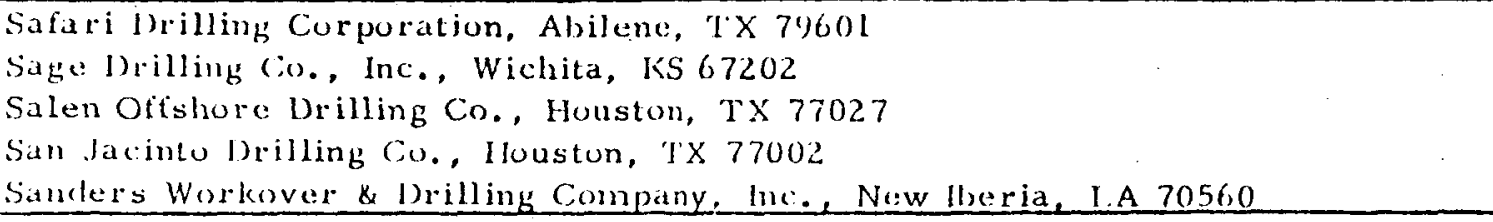 & $\begin{array}{l}6 \\
5 \\
2\end{array}$ & & 2 & $1+1 W$ \\
\hline 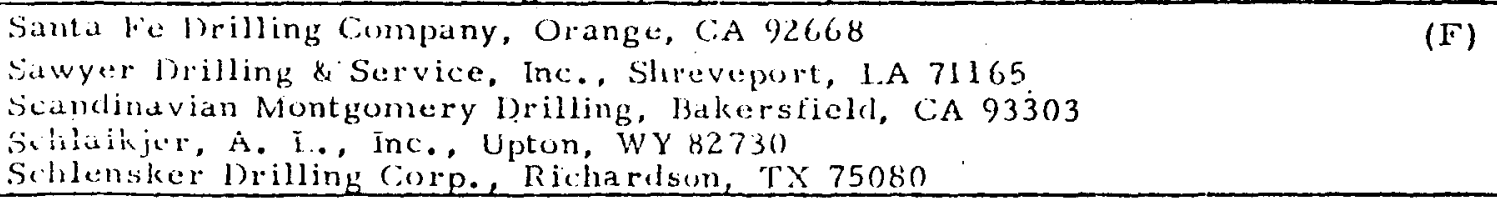 & $\begin{array}{r}34+6 \\
9 \\
4 \\
1 \\
8 \\
\end{array}$ & 3 & 11 & 5 \\
\hline
\end{tabular}


DRILLING COMPANY ANALYSIS, CONTINUED

\begin{tabular}{|c|c|c|c|c|}
\hline \multirow[b]{2}{*}{ COMPANY } & \multicolumn{4}{|c|}{ NUMBER OF RIGS } \\
\hline & $\underset{\mathfrak{I}}{\mathfrak{Z}}$ & $\begin{array}{l}E \\
\stackrel{5}{0} \\
\stackrel{5}{a}\end{array}$ & 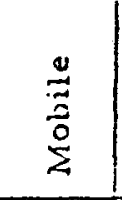 & $\begin{array}{l}0 \\
\dot{3} \\
\stackrel{5}{0} \\
0\end{array}$ \\
\hline $\begin{array}{l}\text { Scott, Don, Drilling Co., Inc., Jonesville, MI } 49250 \\
\text { Scott, Jerry, Drilling Company, Inc., Seminole, OK } 74868 \\
\text { Sea Drilling Corporation, New Orleans, LA } 70112 \\
\text { Seaboard Well Service. Inc. I. afayette, LA } 70505 \\
\text { Search Drilling Company. Wichita, KS } 67218\end{array}$ & \begin{tabular}{l|}
1 \\
4 \\
$5 W$ \\
1
\end{tabular} & 2 & 3 & \\
\hline $\begin{array}{l}\text { Sedco, Inc., Dallas, TX } 75201 \text { (F-46, I.and, } 2 \mathrm{~W}, 19 \text { Mobile) } \\
\text { Service Drilling Company, Tulsa, OK } 74119 \\
\text { Services, Equipment \& Engineering, Inc., Houma, LA } 70360 \\
\text { Shaft Drillers, Inc., Spokane, WA } 99202 \\
\text { Sharp Drilling Company, Inc., Midland, TX } 79702\end{array}$ & \begin{tabular}{l|}
10 \\
18
\end{tabular} & $4 \mathrm{~W}$ & 2 & \\
\hline $\begin{array}{l}\text { Shields Drilling Co., Inc., Russell, KS } 67665 \\
\text { Shirer Well Servicing Co., Inc.. Hoisington, KS } 67544 \\
\text { Shuler Drilling Co., El Dorado, AR } 71730 \\
\text { Signal Drilling Company, Denver, CO } 80202 \\
\text { Sioux Drlg. Corp., Odessa, TX } 79760 \\
\end{array}$ & $\begin{array}{r}1 \\
4 \\
3 \\
12\end{array}$ & & & \\
\hline $\begin{array}{l}\text { Sitton Drilling Company, Lubbock, TX } 79401 \\
\text { Sitton, C. W., Drilling Co, , Inc., Evansville, IN } 47708 \\
\text { Slawson Drilling Co., Inc., Wichita, KS } 67202 \\
\text { Sledge, Gene, Drilling Corp. . Midland, TX } 79701 \\
\text { Snycler Drilling-Well Servicing Co., Grayville, IL } 62844\end{array}$ & $\begin{array}{l}3 \\
5 \\
3 \\
4 \\
1\end{array}$ & & & \\
\hline $\begin{array}{l}\text { Soape Drilling Company, Billings, MT } 59103 \\
\text { Sojourner Drilling Corp., Abilene, TX } 79604 \\
\text { Southland Drilling Co. Inc., Houston, TX } 77024 \\
\text { South Texas Drilling Company, San Antonio, TX } 78205 \\
\text { Standard Drilling Co., Inc., Oklatoma Cily, OK } 73101 \\
\end{array}$ & $\begin{array}{r}1 \\
5 \\
9 \\
3 \\
3+25\end{array}$ & & & \\
\hline 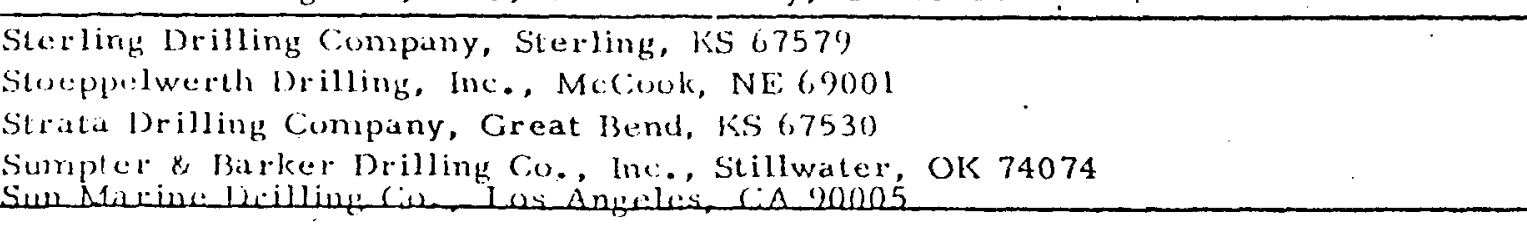 & $\begin{array}{c}3+1 \\
1 \\
1 \\
1\end{array}$ & & 1 & \\
\hline
\end{tabular}




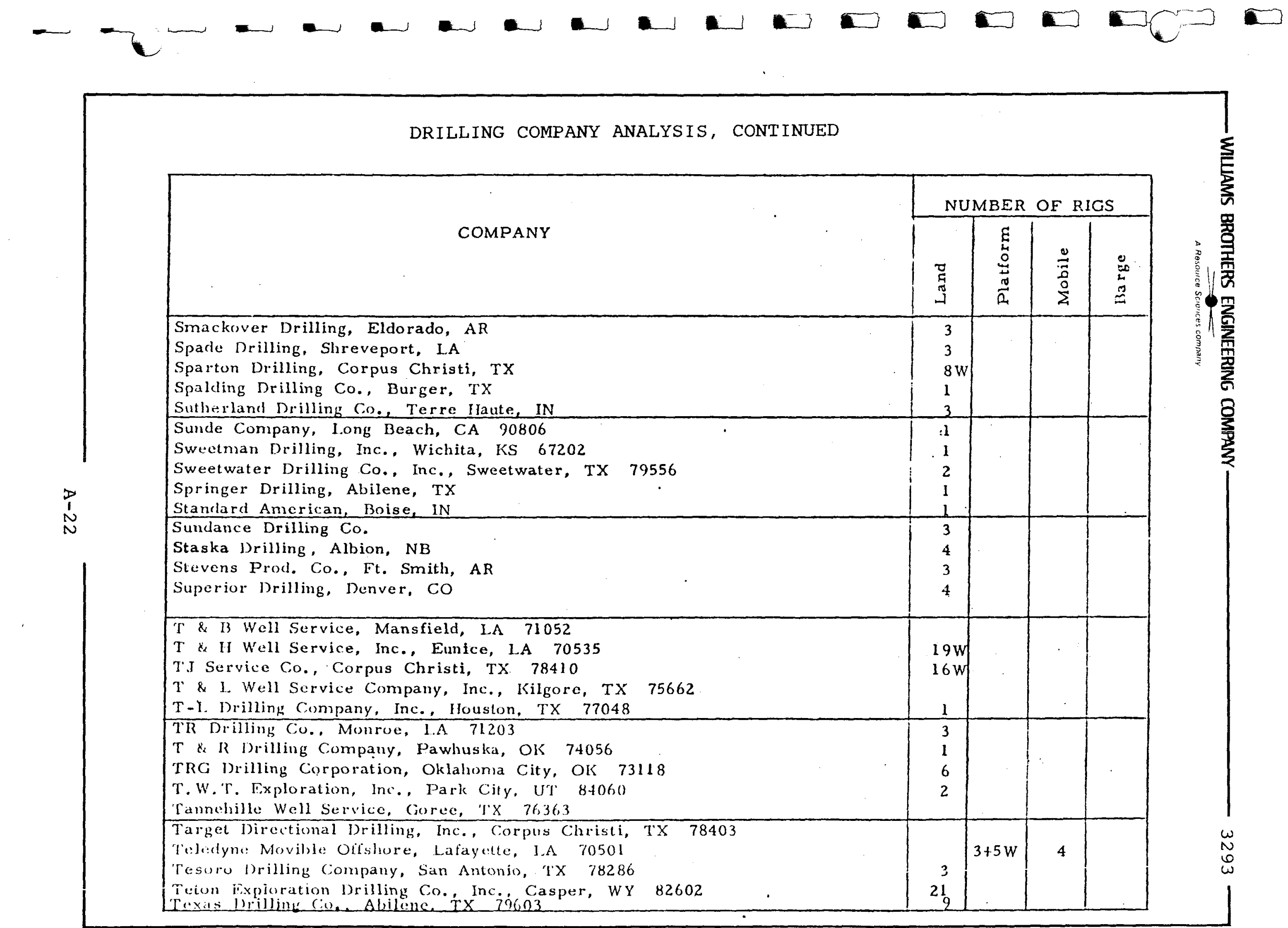


DRILLING COMPANY ANALYSIS, CONTINUED

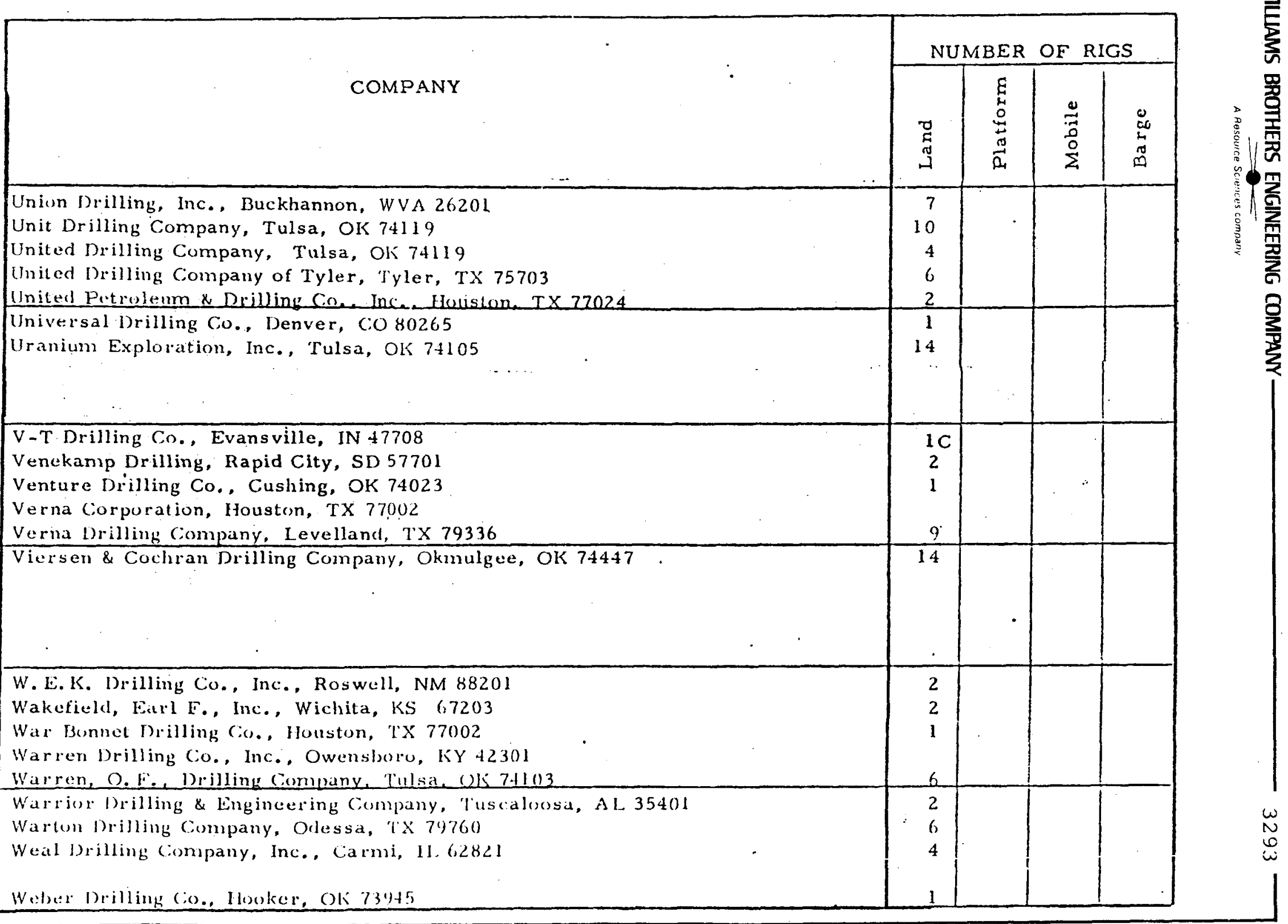




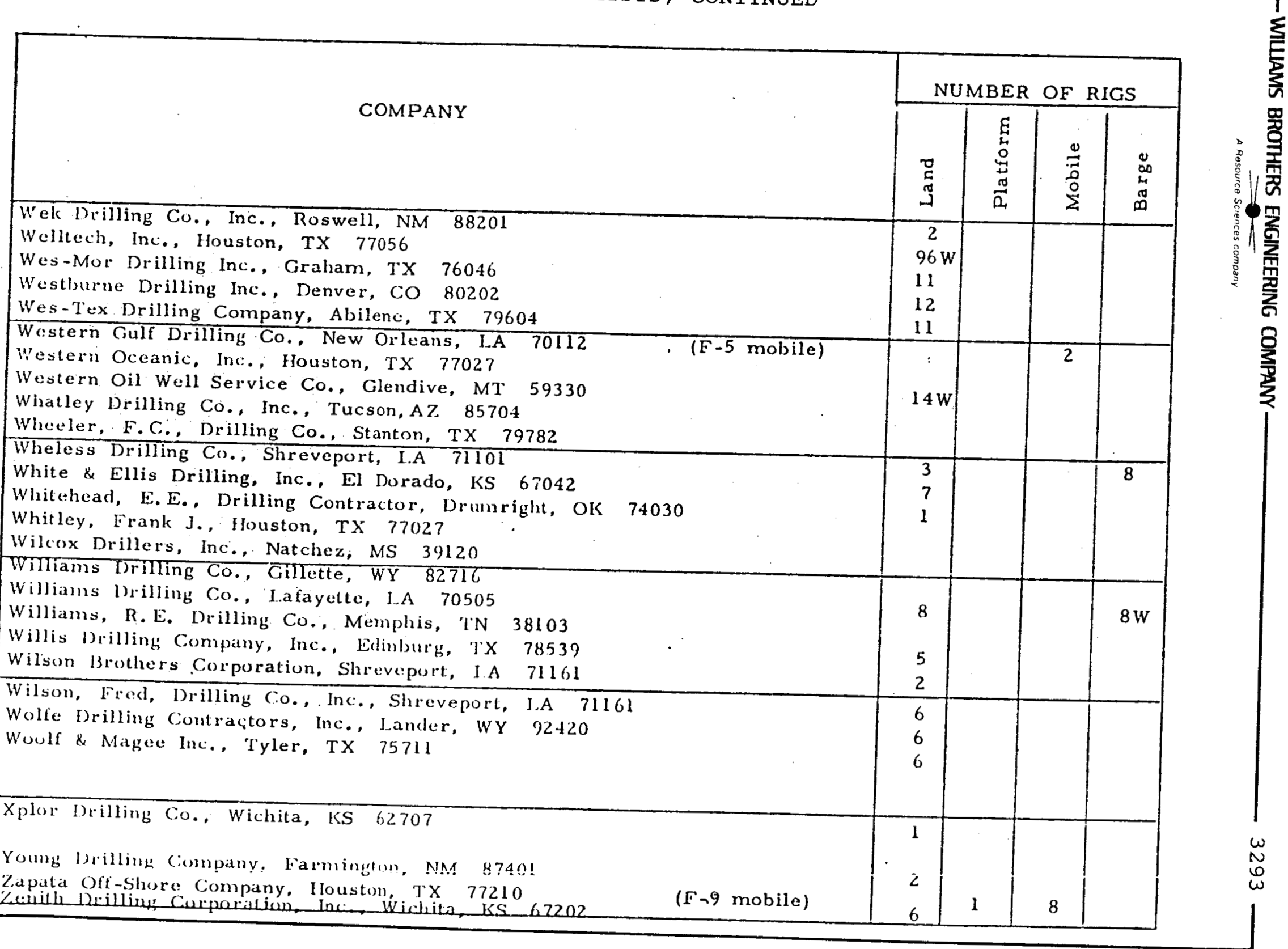




\section{APPENDIX B \\ RELATIONSHIP OF FOOTAGE TO PRODUCTION}

Domestic petroleum production in the United states has declined since 1970. Combined with a steady increase in demand, this decline has created an ever widening gap between supply and demand that must be filled by imported oil (Figure B-1).

In recent years, the only significant input to United states supplies has been production from the North Slope of Alaska. However, North Slope production has not lessened the gap between domestic supply and demand. This gap makes the nation vulnerable to imposition of oil embargoes between 1978 and 1995. The Drilling Technology Program proposed in this document covers this time period. It offers a way to reduce the nation's vulnerability to oil embargoes that are critical to our current economy.

Virtually all the oil and gas produced in the United states is produced from holes that must be drilled into the ground. Historical analysis shows that increased drilling results in increased reserves of oil and gas being found, which leads to increased oil and gas production (Table B-1). The proposed Drilling Technology Program, using the rigs already in operation, can improve oil supply by increasing the productivity of the drilling industry.

It is important to note that increasing the drilling rate does not provide oil and gas reserves or production additional to ultimate reserves. Additional drilling only provides the opportunity to increase production in the near future. In 


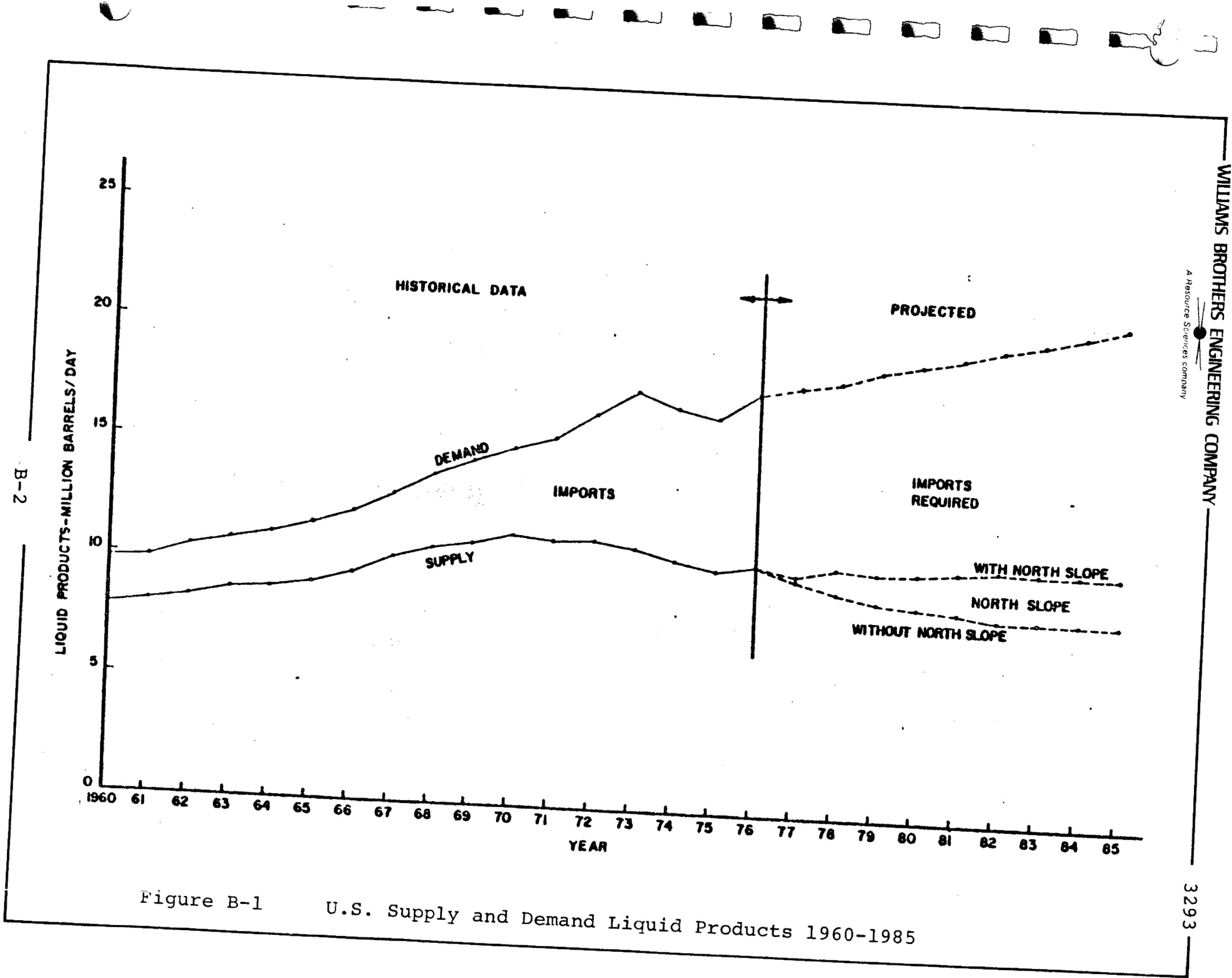




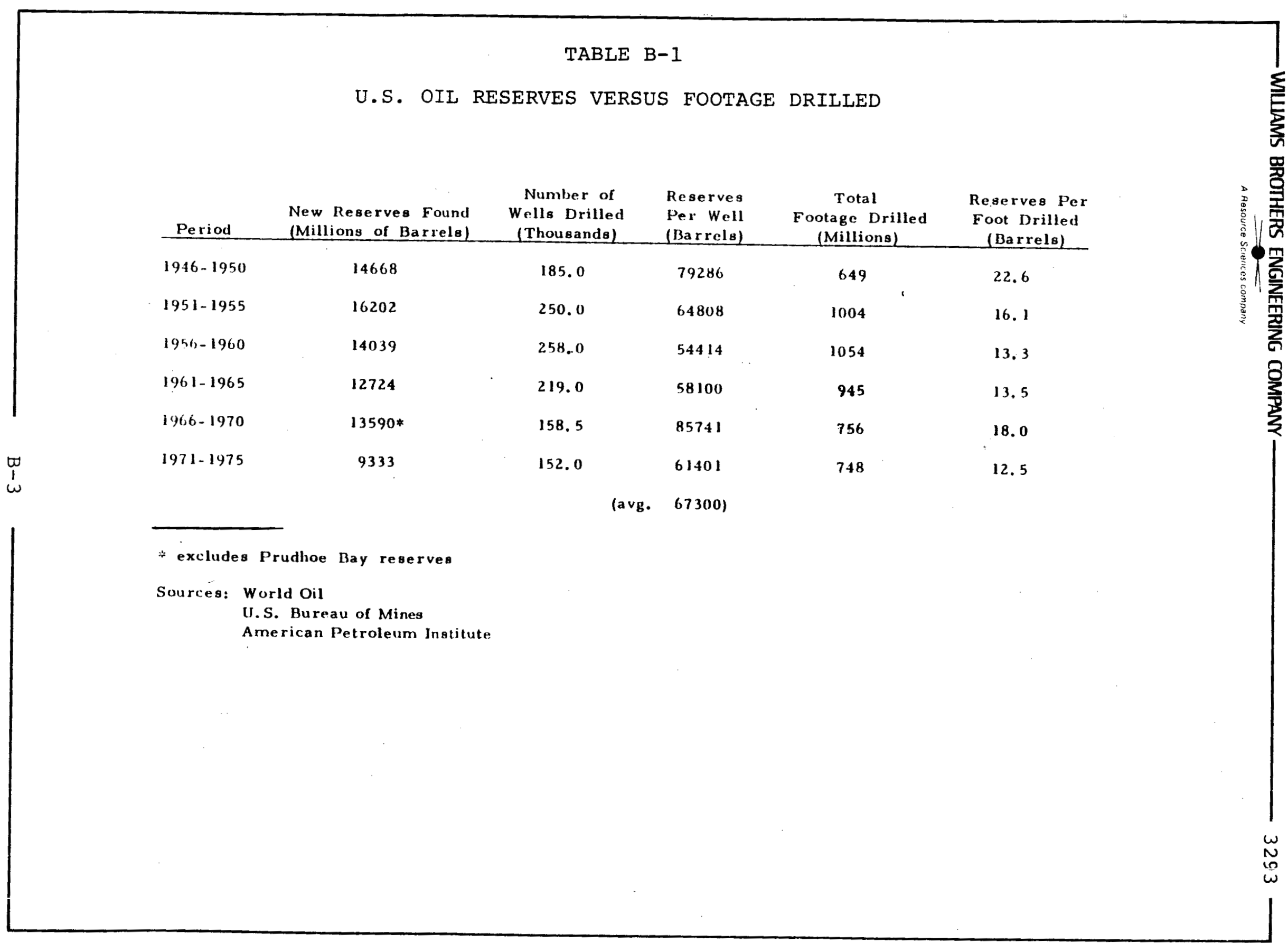


this respect, additional drilling differs from an enhanced oil recovery program. An enhanced oil recovery program provides an increase in the ultimate reserves of oil by allowing the production of a greater percentage of the known oil in-place within reservoirs. For instance, increasing production from 30 percent of the oil in-place to 36 percent of the oil in-place in a reservoir by an enhanced recovery technique provides 20 percent more oil from that reservoir than could be recovered without the enhanced technique.

A review was made to determine the number of projects applicable simultaneously to individual rigs and the resulting effect of project application to productivity.

Table B-2 indicates mutually exclusive projects and groups from which only one project can be used at a time.

In addition to the exclusivity factor, a factor to reduce the overall effectiveness of simultaneous operating projects must be applied. This factor, the Multiple Program Factor (MPF), provides the reduction to compensate for inherent dependencies which would reduce overall effectiveness. Table B-3 shows compatible projects with the projected number of rigs to which they can be applied. The MPF is determined from this data and is applied in Table B-4.

Yearly tables, excluding training, are multiplied by the respective yearly MPF and the product determined is shown in Table B-4. Percent of improvement from Training Project 1-1 is added to obtain the expected improvement. 
TABLE $B-2$

MUTUALLY EXCLUSIVE TECHNOLOGIES

1-1 Training

2-1 Upgrade Conventional Drilling Rigs

2-2 Drilling Rig Automation

2-3 Directional Drilling Equipment

2-4 Bottom-Hole Assemblies

2-5 Mud Automation systems

3-1 Rig Instrumentation/Sensing Equipment

3-2 Rig Floor Display/Analysis Equipment

3-3 Down-Hole Logging Telemetry system

3-4 Surface Logging Telemetry system

3-5 Computer Controlled Drilling

4-1 Jet Erosion Drilling

5-1 Down-Hole Motor Technology

6-1 Stratapax and Bit Technology

7-1 Blowout Preventer

8-1 Advanced Drilling Equipment
Applies to all projects.

Mutually exclusive.

Mutually exclusive.

Applies to all projects.

Not Applicable.

Mutually exclusive.

Applies to all project.s. Mutually exclusive.

Applies to all projects. Not applicable.

Not applicable. 


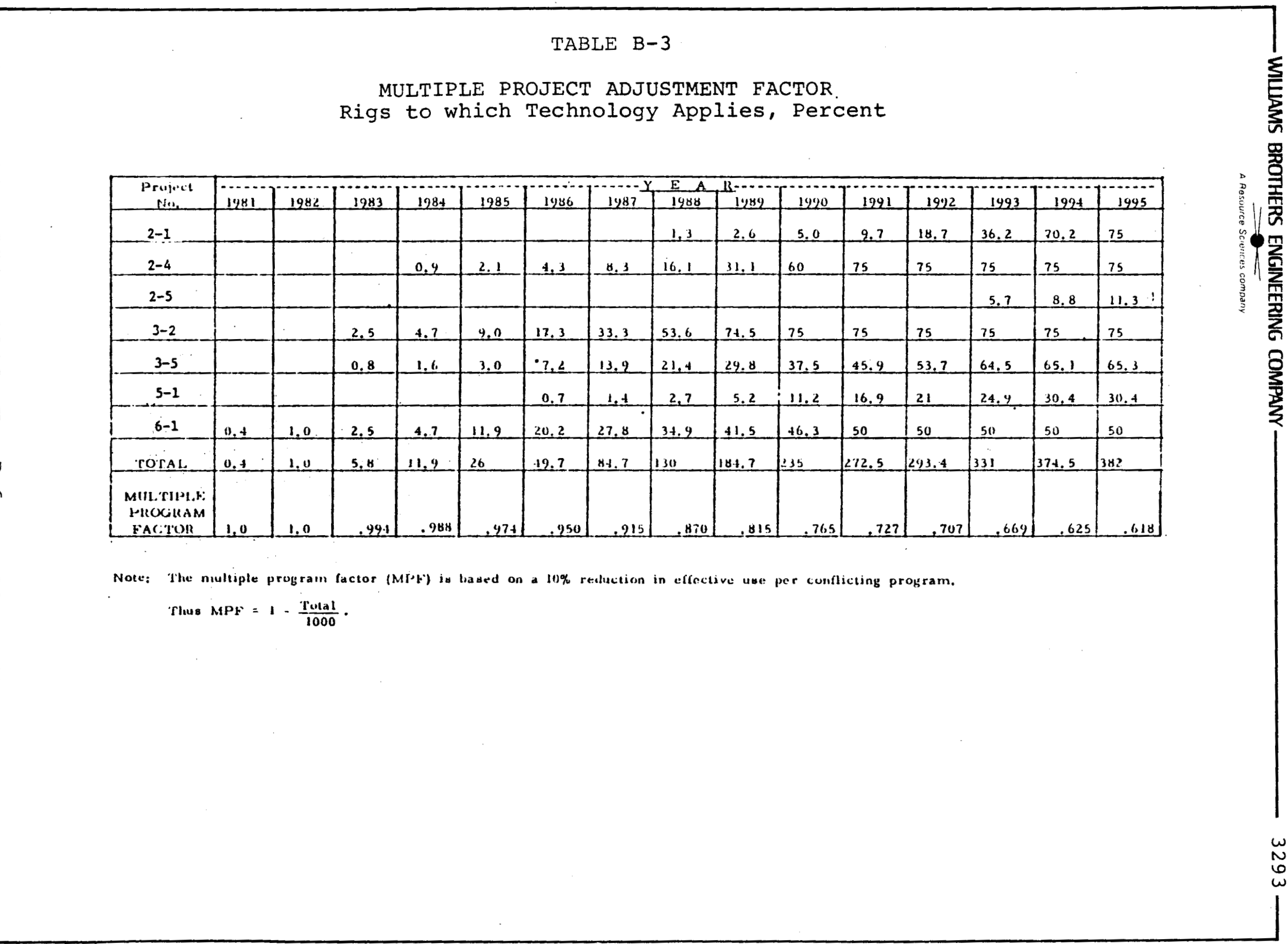




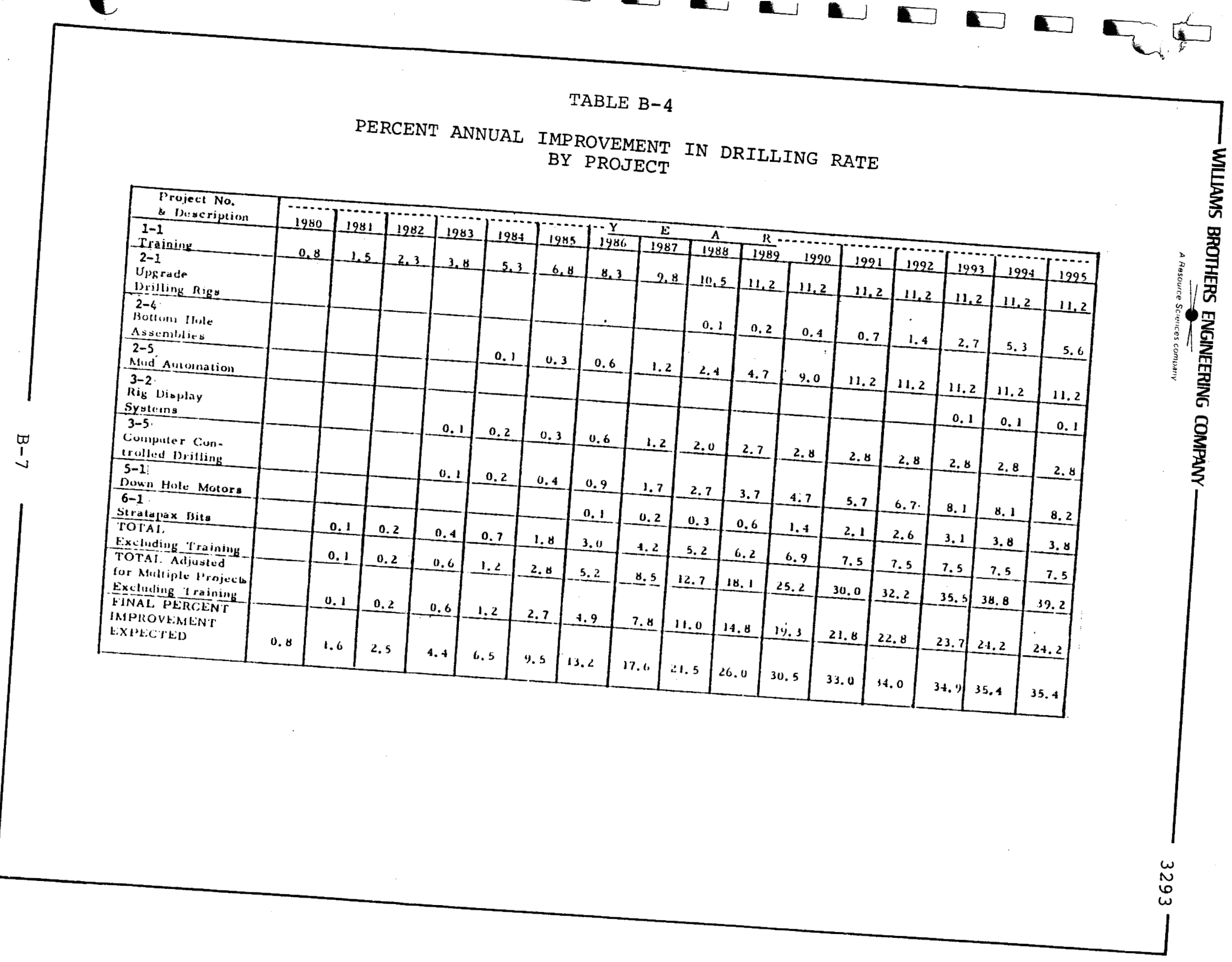

